\title{
INDEX FORMULAS FOR ELLIPTIC BOUNDARY VALUE PROBLEMS IN PLANE DOMAINS WITH CORNERS
}

\author{
GREGORY ESKIN
}

\begin{abstract}
We derive the conditions for the operator corresponding to a general elliptic boundary value problem in a plane domain with corners to be Fredholm and give an explicit formula for the index of this operator.
\end{abstract}

\section{INTRODUCTION}

Let $\mathscr{D}$ be a bounded domain in $\mathbf{R}^{2}$ such that the boundary $\partial \mathscr{D}$ is a closed continuous piecewise smooth curve without self-intersections consisting of $N$ smooth curved segments $\Gamma_{k}, k=1, \ldots, N$, counted counterclockwise with respect to $\mathscr{D}$. Consider in $\mathscr{D}$ an elliptic equation of order $2 m$ :

$$
A(x, D) u(x)=f(x) \text {, }
$$

where

$$
\begin{gathered}
x=\left(x_{1}, x_{2}\right), \quad D=\left(i \frac{\partial}{\partial x_{1}}, i \frac{\partial}{\partial x_{2}}\right), \\
A(x, \xi)=\sum_{k_{1}+k_{2}=0}^{2 m} a_{k_{1} k_{2}}(x) \xi_{1}^{k_{1}} \xi_{2}^{k_{2}}, \quad a_{k_{1} k_{2}}(x) \in C^{\infty}(\overline{\mathscr{D}}) .
\end{gathered}
$$

The ellipticity means that $A_{0}(x, \xi) \neq 0$ for all $x \in \overline{\mathscr{D}},\left(\xi_{1}, \xi_{2}\right) \neq(0,0)$, where $A_{0}(x, \xi)$ is the principal part of $A(x, \xi)$.

On $\partial \mathscr{D}$ we shall consider boundary conditions

$$
\left.B_{k j}(x, D) u\right|_{\Gamma_{k}}=h_{k j}\left(x^{\prime}\right), \quad x^{\prime} \in \Gamma_{k}, 1 \leq j \leq m, 1 \leq k \leq N,
$$

where $\operatorname{deg} B_{k j}=m_{k j}$.

We shall assume that $B_{k j}(x, \xi), 1 \leq j \leq m$, satisfy the Shapiro-Lopatinsky condition on $\bar{\Gamma}_{k}, 1 \leq k \leq N$. To formulate the Shapiro-Lopatinsky condition we shall introduce local coordinates $\left(y_{1}^{(k)}, y_{2}^{(k)}\right)$ in a neighborhood of $\bar{\Gamma}_{k}$ in $\mathbf{R}^{2}$ such that $y_{2}^{(k)}=0$ when $\left(y_{1}^{(k)}, y_{2}^{(k)}\right) \in \bar{\Gamma}_{k}$ and $y_{2}^{(k)}>0$ when $\left(y_{1}^{(k)}, y_{2}^{(k)}\right) \in \mathscr{D}$, $y_{2}^{(k)}$ is small enough, and $\left(y_{1}^{(k)}, 0\right) \in \Gamma_{k}$. Let $A_{k 0}\left(y^{(k)}, \eta^{(k)}\right), B_{k j 0}\left(y^{(k)}, \eta^{(k)}\right)$

Received by the editors January 22, 1988.

1980 Mathematics Subject Classification (1985 Revision). Primary 35J40.

Partially supported by National Science Foundation Grant DMS8502326. 
be the principal parts of $A(x, \xi), B_{k j}(x, \xi)$ in this local system of coordinates. Here $\eta^{(k)}=\left(\eta_{1}^{(k)}, \eta_{2}^{(k)}\right)$ are dual coordinates to $y^{(k)}=\left(y_{1}^{(k)}, y_{2}^{(k)}\right)$. Let

$$
A_{k 0}\left(y^{(k)}, \eta_{1}^{(k)}, w\right)=A_{k 0}^{-}\left(y^{(k)}, \eta_{1}^{(k)}, w\right) A_{k 0}^{+}\left(y^{(k)}, \eta_{1}^{(k)}, w\right)
$$

be the factorization of $A_{k 0}\left(y^{(k)}, \eta^{(k)}\right)$ with respect to $\eta_{2}^{(k)}$, i.e., $\operatorname{Im} w<0$ for all roots of $A_{k 0}^{+}\left(y^{(k)}, \eta_{1}^{(k)}, w\right)$ and $\operatorname{Im} w>0$ for all roots of $A_{k 0}^{-}\left(y^{(k)}, \eta_{1}^{(k)}, w\right)$ where $\eta_{1}^{(k)} \neq 0$ and $\operatorname{deg} A_{k 0}^{+}=\operatorname{deg} A_{k 0}^{-}=m$. Elliptic polynomials satisfying (1.3) are called properly elliptic. Denote

$$
b_{k j r}^{(0)}\left(y_{1}^{(k)}, \eta_{1}^{(k)}\right)=\frac{1}{2 \pi i} \int_{\Gamma_{+}} \frac{B_{k j 0}\left(y_{1}^{(k)}, 0, \eta_{1}^{(k)}, w\right) w^{r-1} d w}{A_{k 0}^{+}\left(y_{1}^{(k)}, 0, \eta_{1}^{(k)}, w\right)},
$$

where $\Gamma_{+}$is a contour in the lower half-plane containing all zeros of

$$
A_{k 0}^{+}\left(y_{1}^{(k)}, \eta_{1}^{(k)}, w\right)
$$

The Shapiro-Lopatinsky condition means

$$
\operatorname{det}\left\|b_{k j r}^{(0)}\left(y_{1}^{(k)}, \eta_{1}^{(k)}\right)\right\|_{j, r=1}^{m} \neq 0
$$

when $\eta_{1}^{(k)} \neq 0,\left(y_{1}^{(k)}, 0\right) \in \bar{\Gamma}_{k}, 1 \leq k \leq N$. Matrix (1.4) is called the Lopatinsky matrix.

We shall consider at first the case when all zeros of $A_{k 0}\left(y^{(k)}, \eta^{(k)}\right)$ are simple, $1 \leq k \leq N$, i.e.,

$$
A_{k 0}\left(y^{(k)}, \eta^{(k)}\right)=a_{0}^{(k)}\left(y^{(k)}\right) \prod_{j=1}^{2 m}\left(\eta_{2}^{(k)}-\lambda_{k j}\left(y^{(k)}\right) \eta_{1}^{(k)}\right),
$$

where

$$
\operatorname{Im} \lambda_{k j}\left(y^{(k)}\right)<0, \quad 1 \leq j \leq m, \quad \operatorname{Im} \lambda_{k j}\left(y^{(k)}\right)>0, \quad m+1 \leq j \leq 2 m .
$$

Then

$$
A_{k 0}^{+}\left(y^{(k)}, \eta^{(k)}\right)=\prod_{j-1}^{m}\left(\eta_{2}^{(k)}-\lambda_{k j}\left(y^{(k)}, \eta_{1}^{(k)}\right)\right),
$$

where $\lambda_{k j}\left(y^{(k)}, \eta_{1}^{(k)}\right)=\lambda_{k j}\left(y^{(k)}\right) \eta_{1}^{(k)}$ when $\eta_{1}^{(k)}>0$ and $\lambda_{k j}\left(y^{(k)}, \eta_{1}^{(k)}\right)=$ $\lambda_{k, j+m}\left(y^{(k)}\right) \eta_{1}^{(k)}$ when $\eta_{1}^{(k)}<0$. In this case the Shapiro-Lopatinsky condition is equivalent to the condition

$$
\begin{aligned}
\operatorname{det}\left\|B_{k j 0}\left(y_{1}^{(k)}, 0, \eta_{1}^{(k)}, \lambda_{k p}\left(y_{1}^{(k)}, 0, \eta_{1}^{(k)}\right)\right)\right\|_{j, p=1}^{m} \neq 0, & \\
& \eta_{1}^{(k)} \neq 0,\left(y_{1}^{(k)}, 0\right) \in \bar{\Gamma}_{k}, 1 \leq k \leq N .
\end{aligned}
$$

In the last section we shall extend all results to the case of arbitrary properly elliptic polynomials. We shall study the boundary value problem (1.1), (1.4) in 
the Sobolev spaces with weights as in $[3, \S 24]$. Denote by $H_{s}\left(\mathbf{R}^{2}\right)$ the Sobolev space with a norm

$$
\|u\|_{s}^{2}=\int_{\mathbf{R}^{2}}\left(1+|\xi|^{2}\right)^{s}|\tilde{u}(\xi)|^{2} d \xi
$$

where

$$
\tilde{u}(\xi)=\int_{\mathbf{R}^{2}} u(x) e^{i(x, \xi)} d x
$$

is the Fourier transform of $u(x)$. As usual $\stackrel{\circ}{H}_{s}(\mathscr{D})$ means the subspace of $H_{s}\left(\mathbf{R}^{2}\right)$ consisting of functions with the support in $\overline{\mathscr{D}}$ and $H_{s}(\mathscr{D})$ means the restrictions of functions in $H_{s}\left(\mathbf{R}^{2}\right)$ to the domain $\mathscr{D}$ with the norm

$$
\|f\|_{s}^{+}=\inf _{l}\|l f\|_{s},
$$

where $f$ is a function (distribution) in $\mathscr{D}, l f$ is an arbitrary extension of $f$ to $\mathbf{R}^{2}$ belonging to $H_{s}\left(\mathbf{R}^{2}\right)$, and the infimum is taken over all extensions of $f$. Consider some imbedding of $\bar{\Gamma}_{k}$ in $\mathbf{R}^{1}$. Then $H_{s}\left(\Gamma_{k}\right)$ means the Sobolev space on $\Gamma_{k}$ consisting of distributions $h$ extendible to $\mathbf{R}^{1}$ with the norm

$$
[h]_{s}^{+}=\inf _{l}[l h]_{s},
$$

where $l h$ is an arbitrary extension of $h$ to $\mathbf{R}^{1}$ and $[l h]_{s}$ is the norm in $H_{s}\left(\mathbf{R}^{1}\right)$. Denote by $P_{1}, P_{2}, \ldots, P_{N}$ the vertices of the domain $\mathscr{D}$; i.e., $P_{k-1}=\bar{\Gamma}_{k-1} \cap$ $\bar{\Gamma}_{k}, 1 \leq k \leq N, \Gamma_{0}=\Gamma_{N}, P_{0}=P_{N}$. Introduce in a neighborhood of $P_{k}$ in $\mathbf{R}^{2}$ a local system of coordinates $\left(x_{1}^{(k)}, x_{2}^{(k)}\right)$ with the origin at $P_{k}$. Denote by $r_{j}\left(x_{1}, x_{2}\right)$ a complex-valued $C^{\infty}$-function in $\mathbf{R}^{2}$ such that $r_{j}\left(x_{1}, x_{2}\right) \neq$ 0 outside of $P_{1}, \ldots, P_{N}, r_{j}=1$ for large $\left(x_{1}, x_{2}\right)$, and $r_{j}\left(x_{1}, x_{2}\right)$ has the following form in local coordinates over $P_{k}$ :

$$
r_{j}=\left(x_{1}^{(k)}+i x_{2}^{(k)}\right)^{j} r_{j k 0}\left(x_{1}^{(k)}, x_{2}^{(k)}\right),
$$

where $r_{j k 0} \neq 0$ and $r_{j k 0} \in C^{\infty}$. We shall denote by $H_{s, N_{1}}\left(\mathbf{R}^{2}\right)$ the space of functions with a norm

$$
\|u\|_{s, N_{1}}=\sum_{j=0}^{N_{1}}\left\|r_{j} u\right\|_{s+j}
$$

where $r_{0}=1$. The Sobolev spaces $\stackrel{\circ}{H}_{s, N_{1}}(\mathscr{D})$ and $H_{s}(\mathscr{D})$ are defined analogously to $\stackrel{\circ}{H}_{s}(\mathscr{D})$ and $H_{s}(\mathscr{D})$. Let $\rho_{j}$ be the restriction of $r_{j}\left(x_{1}, x_{2}\right)$ to $\partial \mathscr{D}$. Denote by $H_{s, N_{1}}\left(\Gamma_{k}\right)$ the space of functions on $\Gamma_{k}$ with a norm

$$
[h]_{s, N_{1}}^{+}=\sum_{j=0}^{N_{1}}\left[\rho_{j} h\right]_{s+j}^{+} .
$$


Let $s$ be some real number. We shall look for the solution of the boundary value problem (1.1), (1.2) in $H_{s, N_{1}}(\mathscr{D})$ assuming that $f \in H_{s-2 m, N_{1}}(\mathscr{D})$ and $h_{k j} \in$ $H_{s-m_{k j}-1 / 2, N_{1}}\left(\Gamma_{k}\right)$. In order to have well-defined restrictions of $B_{k j}(x, D) u$ to $\Gamma_{k}$ we shall assume that

$$
\begin{gathered}
s+N_{1}>\max _{k, j}\left(m_{k j}+\frac{1}{2}\right), \\
s \text { is not an integer if } s \leq \max _{k, j}\left(m_{k j}+\frac{1}{2}\right) .
\end{gathered}
$$

(If $f=0$ in (1.1) then restrictions of $B_{k j}(x, D) u$ to $\Gamma_{k}$ exist for any $s$ (see Proposition 3.1). Then we do not need (1.15), (1.16) and can choose any $N_{1} \geq 0$.) and

It follows from Lemma 24.5 of [3] that the restriction $\left.B_{k j}(x, D) u\right|_{\Gamma_{k}}$ exists

$$
\left[\left.B_{k j}(x, D) u\right|_{\Gamma_{k}}\right]_{s-m_{k j}-1 / 2, N_{1}}^{+} \leq C\|u\|_{s, N_{1}}^{+},
$$

if conditions (1.15), (1.16) are satisfied. Note that we always can take a minimal integer $N_{1}$ satisfying (1.15), in particular $N_{1}=0$ if $s>\max _{k, j}\left(m_{k j}+\frac{1}{2}\right)$. The boundary value problem (1.1), (1.2) defines a bounded operator $\mathscr{A}_{s}$ acting from $H_{s, N_{1}}(\mathscr{D})$ to

$$
\mathscr{H}_{s, N_{\mathrm{l}}}=H_{s-2 m, N_{\mathrm{l}}}(\mathscr{D}) \times \prod_{k, j} H_{s-m_{k j}-1 / 2, N_{\mathrm{l}}}\left(\Gamma_{k}\right)
$$

(see $\S 24$ of [3] for estimates in weight norms (1.13), (1.14)). We shall prove

Theorem 1.1. Assume that (1.5) holds. Then for all $s$ except a discrete set $\Sigma_{B}$ the operator $\mathscr{A}_{s}$ is Fredholm; i.e., $\operatorname{ker} \mathscr{A}_{s}$ is a finite-dimensional subspace of $H_{s, N_{1}}(\mathscr{D})$, range of $\mathscr{A}_{s}$ is closed in $\mathscr{H}_{s, N_{1}} ;$ and coker $\mathscr{A}_{s} \approx \mathscr{H}_{s, N_{1}} \mid \operatorname{Im} \mathscr{A}_{s}$ is finitedimensional where $\operatorname{Im} \mathscr{A}_{s}$ is the range $\mathscr{A}_{s}$. Moreover, $\Sigma_{B}=\bigcup_{k=1}^{N}\left(1-\operatorname{Re} \Sigma_{k-1}\right)$ where $\Sigma_{k-1}$ is the contribution of the vertex $P_{k-1}$, and it is the set of zeros of $\operatorname{det} M_{k-1,0}(z)$ (see (5.48)) in the case of simple roots of $A_{0}(x, \xi)$ and the set of zeros of $\operatorname{det} M_{k-1,0}^{1}(z)$ (see (9.27)) in the general case.

The Fredholm property of elliptic boundary value problems in domains with corners was considered in many papers (see, for example, the recent monograph [6], the survey [7], and [8] and references there). The difference from these works is in the use of spaces $H_{s, N_{1}}$, which are more natural, especially for negative $s$, since $H_{s, N_{\mathrm{t}}}(\mathscr{D})$ is contained in the space of extendible distributions in $\mathscr{D}$ (see also [2], where Sobolev's spaces of noninteger positive order are used, and [9]). The main result of the paper is the formula for the index of $\mathscr{A}_{s}$. Since solutions of boundary value problems in domains with corners have singularities, the index depends on the class of functions in which we consider the problem, i.e., of $s$ where $H_{s}$ is the corresponding Sobolev space. When we enlarge the class then the kernel of the corresponding operator $\mathscr{A}_{S}$ increases and the cokernel decreases. $\Sigma_{B}$ is the exceptional discrete set of $s$ where the 
index jumps and $\mathscr{A}_{s}$ fails to be Fredholm. We shall give formulas that makes this dependence on $s$ explicit.

Theorem 1.2. Index of the operator $\mathscr{A}_{s}$ is given by the formula (5.50) in the case of simple roots of $A_{0}(x, \xi)$ and by the formula (9.10) in the general case. When the principal symbol $A_{0}(x, \xi)$ is real valued one has more explicit formulas (6.37) in the case of simple roots and (9.33) in the general case. For the second-order equation with real coefficients the formula for the index has the form (6.33).

The plan of the paper is the following: In $\S 2$ we find an explicit solution of the homogeneous boundary value problem in an infinite corner. The necessary and sufficient condition for this solution to be bounded in the Sobolev space is called the "corner condition" and we find an explicit form of this condition (see $(2.73)$ ). In $\S 3$ we prove the uniqueness of the solution constructed in $\S 2$. Although the uniqueness result is not used in this paper, it gives an alternative way to prove Theorem 1.1 and it allows one to obtain the asymptotic expansion of the solution near vertices. Also, Proposition 3.1 in $\S 3$ allows one to replace weighted Sobolev spaces by the usual Sobolev space in the case when $f=0$ in (1.1). In $\S 4$ we start to construct a right regularizer (parametrix) for operator $\mathscr{A}_{s}$. We introduce the operator $R_{s}^{(1)}$, which is basically the sum of the parametrix of $A(x, D)$ in $\mathbf{R}^{2}$ and double layer potentials. Replacing $\mathscr{A}_{s}$ by the operator $\mathscr{A}_{s}^{(1)}$ having the same index, we compute the composition $\mathscr{A}_{s}^{(1)} R_{s}^{(1)}=\Phi_{s}$. Operator $\Phi_{s}$ is studied in $\S 5$. It belongs to an algebra of operators similar to the one studied in $[3, \S 15]$ (see also [1]). We find the conditions when $\Phi_{s}$ is Fredholm and compute its index. In $\S 6$ we simplify the formula for the index of $\Phi_{s}$, making it more explicit. In particular we consider separately the case of a second-order elliptic equation. In $\S 7$ we prove that operator $\mathscr{A}_{s}^{(1)}$ (and therefore $\mathscr{A}_{s}$ ) has a finite-dimensional kernel. This completes the prove of Theorem 1.1. Also we prove in $\S 7$ that the operator $R_{s}^{(1)}$ is Fredholm for all $s \in \mathbf{R}$ and its index is equal to zero: ind $R_{s}^{(1)}=0$. Therefore ind $\mathscr{A}_{s}=$ ind $\Phi_{s}$ and results of $\S \S 5$ and 6 allow us to obtain the formula for ind $\mathscr{A}_{s}$. In $\S 8$ we consider examples of boundary value problems for the Laplacian and also some regularity results. In $\S 9$ we remove the restriction made in previous sections that the symbol of the elliptic operator has only simple roots.

Most of the methods used in the present paper are similar to those in the author's previous work [3-5].

\section{MODEL PROBLEM IN A CORNER}

Let $G$ be a corner in $\mathbf{R}^{2}$ formed by the semiaxis $\Gamma_{1}=\left\{x_{1}>0, x_{2}=0\right\}$ and the semiaxis $\Gamma_{2}=\left\{y_{1}=-x_{1} \cos \alpha-x_{2} \sin \alpha<0, y_{2}=x_{1} \sin \alpha-x_{2} \cos \alpha=0\right\}$. The interior angle of $G$ counted counterclockwise is equal to $\alpha, 0<\alpha<2 \pi$.

Consider a homogeneous equation in $G$

$$
A_{0}\left(i \frac{\partial}{\partial x_{1}}, i \frac{\partial}{\partial x_{2}}\right) u\left(x_{1}, x_{2}\right)=0
$$


where $A_{0}\left(\xi_{1}, \xi_{2}\right)$ is a properly elliptic homogeneous polynomial of degree $2 m$ having only simple roots

$$
A_{0}\left(\xi_{1}, \xi_{2}\right)=a_{0} \prod_{j=1}^{2 m}\left(\xi_{2}-\lambda_{j} \xi_{1}\right),
$$

where $a_{0} \neq 0$,

$$
\operatorname{Im} \lambda_{j}<0, \quad \operatorname{Im} \lambda_{j+m}>0, \quad 1 \leq j \leq m .
$$

The results of this section play only an auxiliary role and therefore we shall impose boundary conditions of the form that will be used in following sections.

Let

$$
\Lambda_{-}^{1 / 2-s}=\left(i \frac{\partial}{\partial x_{1}} \cos \frac{\alpha}{2}+i \frac{\partial}{\partial x_{2}} \sin \frac{\alpha}{2}-i 0\right)^{1 / 2-s}
$$

be a pseudodifferential operator in $\mathbf{R}^{2}$ with symbol

$$
\Lambda_{-}^{1 / 2-s}\left(\xi_{1}, \xi_{2}\right)=\left(\xi_{1} \cos \frac{\alpha}{2}+\xi_{2} \sin \frac{\alpha}{2}-i 0\right)^{1 / 2-s},
$$

where by definition

$$
(t-i 0)^{1 / 2-s}=\lim _{\varepsilon \rightarrow 0} e^{(1 / 2-s) \ln (t-i \varepsilon)}, \quad t \in \mathbf{R}, \varepsilon>0,
$$

and we consider the branch of $\ln (t-i \varepsilon)$ that is real for $t>0$ and $\varepsilon=0$. In all computations we shall take $\varepsilon>0$ in the beginning and then pass to the limit $\varepsilon=0$ at a later stage when it will cause no problem.

Denote by $\lambda_{1 / 2-s}^{-}$the inverse Fourier transform of the distribution (2.4). Let $x^{1}=U_{\beta} x$ be the rotation of the plane by the angle $\beta$, i.e.,

$$
x_{1}^{1}=x_{1} \cos \beta-x_{2} \sin \beta, \quad x_{2}^{1}=x_{1} \sin \beta+x_{2} \cos \beta .
$$

Since $F^{-1}\left(\xi_{1}-i 0\right)^{1 / 2-s}=C x_{1,-}^{s-3 / 2} \delta\left(x_{2}\right)$ where $x_{1,-}^{s-3 / 2}=0$ for $x_{1}<0$ and $C$ is a constant (see, for example, $[3, \S 2])$ we obtain, using the change of variables (2.5) with $\beta=-\alpha / 2$, that

$$
\lambda_{1 / 2-s}^{-}(x)=C\left(x_{1} \cos \frac{\alpha}{2}+x_{2} \sin \frac{\alpha}{2}\right)_{-}^{s-3 / 2} \delta\left(-x_{1} \sin \frac{\alpha}{2}+x_{2} \cos \frac{\alpha}{2}\right) .
$$

Note that support of $\lambda_{1 / 2-s}^{-}(x)$ belongs to $C G$, where $C G$ is the complement of the open domain $G$. Moreover, if $u_{-}$is a distribution with support in $C G$ then the support of $\Lambda_{-}^{1 / 2-s} u=\lambda_{1 / 2-s}^{-} * u_{-}$is also in $C G$. We shall call an operator $A_{-}$with such a property a "minus"-operator with respect to domain $G$. For "minus"-operator $A_{-}$and a distribution $u$ in $G$ we have that $p_{G} A_{-} l u$ is independent of the choice of the extension $l u$ where $p_{G}$ is the restriction operator to $G$. Note that in (2.4) we can replace $\alpha / 2$ by any $\beta$ such that 
$0 \leq \beta \leq \alpha$ if $\alpha \leq \pi$ and $\alpha-\pi \leq \beta \leq \pi$ if $\alpha>\pi$. We shall consider the following boundary conditions:

$$
\begin{aligned}
& p_{1}^{+}\left(i \frac{\partial}{\partial x_{1}}-i 0\right)^{s-m_{j 1}-1 / 2} B_{j 1}\left(i \frac{\partial}{\partial x_{1}}, i \frac{\partial}{\partial x_{2}}\right) \Lambda_{-}^{1 / 2-s} l u=h_{j 1}\left(x_{1}\right) \\
& p_{2}^{-}\left(-i \frac{\partial}{\partial x_{1}} \cos \alpha-i \frac{\partial}{\partial x_{2}} \sin \alpha+i 0\right)^{s-m_{j 2}-1 / 2} \\
& \cdot B_{j 2}\left(i \frac{\partial}{\partial x_{1}}, i \frac{\partial}{\partial x_{2}}\right) \Lambda_{-}^{1 / 2-s} l u=h_{j 2}\left(y_{1}\right), \quad y_{1}<0,1 \leq j \leq m,
\end{aligned}
$$

where $p_{1}^{+}, p_{2}^{-}$are the restriction operators to $\Gamma_{1}, \Gamma_{2}$, respectively, $B_{j i}\left(\xi_{1}, \xi_{2}\right)$ are homogeneous polynomials of degree $m_{j i}$, and coordinates $\left(y_{1}, y_{2}\right)$ are related to $\left(x_{1}, x_{2}\right)$ by the rotation by the angle $\beta=\pi-\alpha$, i.e.,

$$
y_{1}=-x_{1} \cos \alpha-x_{2} \sin \alpha, \quad y_{2}=x_{1} \sin \alpha-x_{2} \cos \alpha \text {. }
$$

Note that

$$
\left(-i \frac{\partial}{\partial x_{1}} \cos \alpha-i \frac{\partial}{\partial x_{2}} \sin \alpha+i 0\right)^{s-m_{j 2}-1 / 2}=\left(i \frac{\partial}{\partial y_{1}}+i 0\right)^{s-m_{j 2}-1 / 2}
$$

is a "minus"-operator with respect to $\Gamma_{2}$; that is, $p_{\Gamma_{2}}\left(i \partial / \partial y_{1}+i 0\right)^{s-m_{j 2}-1 / 2} l g$ is independent of the choice of the extension $\lg$ from $\Gamma_{2}$ to $\mathbf{R}^{1}$ since if $g_{-}=0$ for $y_{1}>0$ then also $\left(i \partial / \partial y_{1}+i 0\right)^{s-m_{j 2}-1 / 2} g_{-}=0$ for $y_{1}>0$. Analogously $\left(i \partial / \partial x_{1}-i 0\right)^{s-m_{j 1}-1 / 2}$ is a "minus"-operator with respect to $\Gamma_{1}$.

As we shall see below, the solution of the boundary value problem (2.1), (2.7), (2.8) does not belong to $L_{2}(G)$. Therefore in this section we shall modify the Sobolev spaces $H_{s, N_{1}}$ introduced in $\S 1$.

Denote by $H_{1 / 2,0}^{(1)}\left(\mathbf{R}^{2}\right)$ the closure of $C_{0}^{\infty}\left(\mathbf{R}^{2}\right)$ in the norm

$$
\left\|\Lambda_{0}^{1 / 2} u\right\|_{0}^{2}=\int_{\mathbf{R}^{2}}|\xi \| \tilde{u}(\xi)|^{2} d \xi, \quad|\xi|=\sqrt{\xi_{1}^{2}+\xi_{2}^{2}} .
$$

Note that $H_{1 / 2,0}^{(1)}\left(\mathbf{R}^{2}\right)$ is a subspace of the space of tempered distributions $S^{\prime}$ since

$$
|(u, \varphi)|=\frac{1}{(2 \pi)^{n}}\left|\int_{\mathbf{R}^{2}} \tilde{u}(\xi) \overline{\tilde{\varphi}(\xi)} d \xi\right| \leq \frac{1}{(2 \pi)^{n}}\left\|\Lambda_{0}^{1 / 2} u\right\|_{0}\left\|\Lambda_{0}^{-1 / 2} \varphi\right\|_{0}
$$

and $\left\|\Lambda_{0}^{-1 / 2} \tilde{\varphi}\right\|_{0}$ is finite for any $\varphi \in S$.

Note that if $\psi\left(x_{1}, x_{2}\right) \in C_{0}^{\infty}\left(\mathbf{R}^{2}\right)$ then $\psi u \in H_{1 / 2, N_{1}}\left(\mathbf{R}^{2}\right)$ for any $u \in$ $H_{1 / 2, N_{1}}^{(1)}\left(\mathbf{R}^{2}\right)$. Indeed we have

$$
K(\xi, \eta)=(1+|\xi|)^{1 / 2} \tilde{\psi}(\xi-\eta)|\eta|^{-1 / 2}=K_{1}+K_{2},
$$


where

$$
\begin{array}{ll}
K_{1}=K \chi_{0}(\eta), \quad & K_{2}=K\left(1-\chi_{0}(\eta)\right), \quad \chi_{0}(\xi) \in C_{0}^{\infty}\left(\mathbf{R}^{2}\right), \\
& \chi_{0}(\xi)=1 \text { for }|\xi| \leq 1 .
\end{array}
$$

Therefore $K$ is a kernel of an operator bounded in $L_{2}\left(\mathbf{R}^{2}\right)$ since $K_{1}$ is a Hilbert-Schmidt kernel and $\left|K_{2}\right| \leq C /(1+|\xi-\eta|)^{3}$. So for any $v \in C_{0}^{\infty}\left(\mathbf{R}^{2}\right)$

$$
\|\psi v\|_{1 / 2}^{2} \leq C\left\|\Lambda_{0}^{1 / 2} v\right\|_{0}^{2}
$$

and therefore $\psi u \in H_{1 / 2}\left(\mathbf{R}^{2}\right)$ for any $u \in H_{1 / 2,0}^{(1)}\left(\mathbf{R}^{2}\right)$. By $H_{1 / 2, N_{1}}^{(1)}\left(\mathbf{R}^{2}\right)$ we denote a completion of $C_{0}^{\infty}\left(\mathbf{R}^{2}\right)$ in the norm

$$
\left\|\Lambda_{0}^{1 / 2} u\right\|_{0, N_{1}}^{2}=\sum_{k_{1}+k_{2}=0}^{N_{1}}\left\|x_{1}^{k_{1}} x_{2}^{k_{2}} \Lambda_{0}^{1 / 2+k_{1}+k_{2}} u\right\|_{0}^{2} .
$$

Note that (2.12) is equivalent to

$$
\sum_{k_{1}+k_{2}=0}^{N_{1}}\left\|\Lambda_{0}^{1 / 2+k_{1}+k_{2}} x_{1}^{k_{1}} x_{2}^{k_{2}} u\right\|_{0}^{2} \text {. }
$$

The space $H_{1 / 2, N_{1}}^{(1)}(G)$ is defined analogously to (1.10).

Theorem 2.1. Let $\mathscr{A}$ be the operator defined by the left-hand sides of (2.7), (2.8), where $u \in \operatorname{ker} A_{0} \cap H_{1 / 2, N_{1}}^{(1)}(G), \operatorname{ker} A_{0}$ consists of all distributions in $G$ satisfying (2.1). Assume that the Shapiro-Lopatinsky condition (see (2.56)) and the "corner condition" (see (2.73)) are satisfied. Then there exists an operator $R$ bounded from $\mathscr{H}_{0, N_{1}}=\prod_{j=1}^{N} H_{0, N_{1}}\left(\Gamma_{1}\right) \times \prod_{j=1}^{m} H_{0, N_{1}}\left(\Gamma_{2}\right)$ to $\operatorname{ker} A_{0} \cap H_{1 / 2, N_{1}}^{(1)}(G)$ that is the right inverse to $\mathscr{A}$, i.e.,

$$
\mathscr{A} R=I,
$$

where $I$ is the identity operator in $\mathscr{H}_{0, N_{1}}, N_{1} \geq 0$ is arbitrary, and $H_{0, N_{1}}\left(\mathbf{R}^{1}\right)$ is a space with a norm

$$
[g(t)]_{0, N_{\mathrm{I}}}^{2}=\sum_{k=0}^{N_{1}}\left[t^{k} \frac{d^{k}}{d t^{k}} g\right]_{0}^{2} .
$$

Proof. We shall construct $R$ explicitly. First we shall consider the case when $0<\alpha \leq \pi$. The case when $\pi<\alpha<2 \pi$ will be treated in Remark 2.1. Denote by $R_{0}$ the following operator:

$$
\begin{aligned}
u= & R_{0}\left(c_{1}, c_{2}\right)=\sum_{j=1}^{m} \frac{1}{2 \pi} \int_{-\infty}^{\infty} e^{-i x_{2} \lambda_{j}\left(\xi_{1}\right)-i x_{1} \xi_{1}} \tilde{c}_{j 1}\left(\xi_{1}\right) d \xi_{1} \\
& +\sum_{j=1}^{m} \frac{1}{2 \pi} \int_{-\infty}^{\infty} e^{-i y_{2} \mu_{j}\left(\eta_{1}\right)-i y_{1} \eta_{1}} \tilde{c}_{j 2}\left(\eta_{1}\right) d \eta_{1}
\end{aligned}
$$


where

$$
\lambda_{j}\left(\xi_{1}\right)= \begin{cases}\lambda_{j} \xi_{1} & \text { for } \xi_{1}>0 \\ \lambda_{j+m} \xi_{1} & \text { for } \xi_{1}<0\end{cases}
$$

$\mu_{j}\left(\eta_{1}\right)$ will be defined later, and $\left(c_{1}, c_{2}\right)=\left\{c_{j 1}, c_{j 2}\right\} \in \mathscr{K}_{0, N_{1}}, \tilde{c}_{j i}$ are the Fourier transforms of $c_{j i}, c_{j 1}=0$ for $x_{1}<0, c_{j 2}=0$ for $y_{1}>0$. It follows from (2.16) that

$$
A_{0}\left(\xi_{1}, \lambda_{j}\left(\xi_{1}\right)\right)=0, \quad \operatorname{Im} \lambda_{j}\left(\xi_{1}\right)<0, \quad \forall \xi_{1} \neq 0,1 \leq j \leq m .
$$

The symbol of $A_{0}\left(i \partial / \partial x_{1}, i \partial / \partial x_{2}\right)$ has the following form in the coordinates $y=\left(y_{1}, y_{2}\right)(\operatorname{see}(2.9))$ :

$$
A_{0}^{(1)}\left(\eta_{1}, \eta_{2}\right)=a_{0} \prod_{j=1}^{2 m}\left(-\eta_{1} \sin \alpha-\eta_{2} \cos \alpha-\lambda_{j}\left(-\eta_{1} \cos \alpha+\eta_{2} \sin \alpha\right)\right),
$$

since $x=U_{-(\pi-\alpha)} y$ and $\xi=\left(U_{-(\pi-\alpha)}^{*}\right)^{-1} \eta=U_{-(\pi-\alpha)} \eta$. So

$$
A_{0}^{(1)}\left(\eta_{1}, \eta_{2}\right)=a_{0}\left(-\cos \alpha-\lambda_{j} \sin \alpha\right)^{2 m} \prod_{j=1}^{2 m}\left(\eta_{2}-\mu_{j} \eta_{1}\right)
$$

where

$$
\mu_{j}=\frac{\sin \alpha-\lambda_{j} \cos \alpha}{-\cos \alpha-\lambda_{j} \sin \alpha}, \quad 1 \leq j \leq 2 m .
$$

Functions $\mu_{j}\left(\eta_{1}\right)$ in $(2.15)$ have the form

$$
\mu_{j}\left(\eta_{1}\right)= \begin{cases}\mu_{j} \eta_{1} & \text { for } \eta_{1}>0 \\ \mu_{j+m} \eta_{1} & \text { for } \eta_{1}<0\end{cases}
$$

Note that

$$
\operatorname{Im} \mu_{j}=\operatorname{Im} \lambda_{j} /\left|\cos \alpha+\lambda_{j} \sin \alpha\right|^{2},
$$

so that $\operatorname{Im} \mu_{j}\left(\eta_{1}\right)<0$ for $\eta_{1} \neq 0,1 \leq j \leq m$. One can compute integrals in $\xi_{1}$ and $\eta_{1}$ in (2.15):

$$
\begin{aligned}
G_{j 1}\left(x_{2}, x_{1}-t\right)= & \frac{1}{2 \pi} \int_{-\infty}^{\infty} e^{-i x_{2} \lambda_{j}\left(\xi_{1}\right)-i\left(x_{1}-t\right) \xi_{1}} d \xi_{1} \\
= & \frac{1}{2 \pi} \int_{0}^{\infty} e^{-i x_{2} \lambda_{j} \xi_{1}-i\left(x_{1}-t\right) \xi_{1}} d \xi_{1} \\
& +\frac{1}{2 \pi} \int_{-\infty}^{0} e^{-i x_{2} \lambda_{j+m} \xi_{1}-i\left(x_{1}-t\right) \xi_{1}} d \xi_{1} \\
= & \frac{1}{2 \pi i\left(x_{2} \lambda_{j}+x_{1}-t\right)}-\frac{1}{2 \pi i\left(x_{2} \lambda_{j+m}+x_{1}-t\right)}
\end{aligned}
$$


where $t>0,\left(x_{1}, x_{2}\right) \in G$. Analogously

$$
\begin{aligned}
G_{j 2}\left(y_{2}, y_{1}-t\right) & =\frac{1}{2 \pi} \int_{-\infty}^{\infty} e^{-i y_{2} \mu_{j}\left(\eta_{1}\right)-i\left(y_{1}-t\right) \eta_{1}} d \eta_{1} \\
& =\frac{1}{2 \pi i\left(y_{2} \mu_{j}+y_{1}-t\right)}-\frac{1}{2 \pi i\left(y_{2} \mu_{j+m}+y_{1}-t\right)},
\end{aligned}
$$

where $t<0$. Therefore (2.15) can be rewritten in the following form:

$$
\begin{aligned}
u= & R_{0}\left(c_{1}, c_{2}\right)=\sum_{j=1}^{m} \int_{0}^{\infty} G_{j 1}\left(x_{2}, x_{1}-t\right) c_{j 1}(t) d t \\
& +\sum_{j=1}^{m} \int_{-\infty}^{0} G_{j 2}\left(y_{2}, y_{1}-t\right) c_{j 2}(t) d t .
\end{aligned}
$$

Note that in general $u$ does not belong to $L_{2}(G)$ but, as we shall show now, $u \in H_{1 / 2, N_{1}}^{(1)}(G)$. Denote

$$
\begin{aligned}
& u_{j 1}=G_{j 1} c_{j 1} \text { for } x_{2} \geq 0, \\
& u_{j 1}=\frac{1}{2 \pi} \int_{-\infty}^{\infty} e^{-i\left|x_{2}\right| \lambda_{j}\left(\xi_{1}\right)-i x_{1} \xi_{1}} \widetilde{c_{j 1}}\left(\xi_{1}\right) d \xi_{1} \text { for } x_{2}<0
\end{aligned}
$$

that is, $u_{j 1}$ is an extension of $G_{j 1} c_{j 1}$ to $\mathbf{R}^{2}$. Computing the Fourier transform of $u_{j 1}$ in $\left(x_{1}, x_{2}\right)$ we obtain

$$
\begin{aligned}
\widetilde{u_{j 1}}\left(\xi_{1}, \xi_{2}\right) & =i\left(\frac{1}{\xi_{2}-\lambda_{j}\left(\xi_{1}\right)}-\frac{1}{\xi_{2}+\lambda_{j}\left(\xi_{1}\right)}\right) \widetilde{c_{j 1}}\left(\xi_{1}\right) \\
& =\frac{2 i \lambda_{j}\left(\xi_{1}\right)}{\xi_{2}^{2}-\lambda_{j}^{2}\left(\xi_{1}\right)} \widetilde{c_{j 1}}\left(\xi_{1}\right) .
\end{aligned}
$$

Therefore

$$
\begin{aligned}
\left\|\Lambda_{0}^{1 / 2} u_{j 1}\right\|_{0}^{2} & =\int_{\mathbf{R}^{2}} \frac{|\xi|\left|2 \lambda_{j}\left(\xi_{1}\right)\right|^{2}\left|\widetilde{c_{j 1}}\left(\xi_{1}\right)\right|^{2}}{\left|\xi_{2}^{2}-\lambda_{j}^{2}\left(\xi_{1}\right)\right|^{2}} d \xi_{1} d \xi_{2} \\
& \leq C \int_{-\infty}^{\infty}\left|\widetilde{c_{j 1}}\left(\xi_{1}\right)\right|^{2} d \xi_{1}=C\left[c_{j 1}\right]_{0}^{2}
\end{aligned}
$$

Using an extension operator of order $N_{1}+1$ (see, for example, (4.55) in [3]) instead of (2.26) one proves that

$$
\begin{aligned}
\sum_{k_{1}+k_{2}=0}^{N_{1}}\left\|x_{1}^{k_{1}} x_{2}^{k_{2}} \Lambda_{0}^{1 / 2+k_{1}+k_{2}} u_{j 1}\right\|_{0}^{2} & \leq \sum_{k_{1}+k_{2}=0}^{N_{1}}\left\|\frac{\partial^{k_{1}+k_{2}}}{\partial \xi_{1}^{k_{1}} \partial \xi_{2}^{k_{2}}}|\xi|^{1 / 2+k_{1}+k_{2}} \widetilde{u_{j 1}}\right\|_{0}^{2} \\
& \leq C \sum_{k=0}^{N_{1}}\left[\xi_{1}^{k} \frac{\partial^{k}}{\partial \xi_{1}^{k}} \widetilde{c_{j 1}}\left(\xi_{1}\right)\right]_{0}^{2} \leq C\left[c_{j 1}\right]_{0, N_{1}}^{2}
\end{aligned}
$$

Note that if $\widetilde{c_{j 1}}\left(\xi_{1}\right)$ is smooth and $\widetilde{c_{j 1}}(0)=0$ then $u_{j 1} \in H_{1 / 2, N_{1}}\left(\mathbf{R}^{2}\right)$. Therefore for arbitrary $c_{j 1} \in H_{0, N_{1}}\left(\mathbf{R}^{1}\right)$ we can approximate $u_{j 1}$ by functions from 
$H_{1 / 2, N_{1}}\left(\mathbf{R}^{2}\right)$, and since $C_{0}^{\infty}\left(\mathbf{R}^{2}\right)$ is dense in $H_{1 / 2, N_{1}}\left(\mathbf{R}^{2}\right)$ we obtain from (2.29) that $G_{j 1} c_{j 1} \in H_{1 / 2, N_{1}}^{(1)}(G)$. The same arguments apply to $G_{j 2} c_{j 2}$. Therefore we proved that $R_{0}$ is a bounded operator from $\mathscr{H}_{0, N_{1}}$ to $H_{1 / 2, N_{1}}^{(1)}(G)$ for any $N_{1} \geq 0$. Now substitute (2.15) in the boundary conditions (2.7). We obtain (2.30)

$$
\begin{aligned}
& \sum_{k=1} \frac{1}{2 \pi} \int_{-\infty}^{\infty} B_{j 1}\left(\xi_{1}, \lambda_{k}\left(\xi_{1}\right)\right) \Lambda_{-}^{1 / 2-s}\left(\xi_{1}, \lambda_{k}\left(\xi_{1}\right)\right) \cdot\left(\xi_{1}-i 0\right)^{s-m_{j 1}-1 / 2} \widetilde{c_{k_{1}}}\left(\xi_{1}\right) e^{-i x_{1} \xi_{1}} d \xi_{1} \\
&+\sum_{k=1}^{m} \frac{1}{2 \pi} \int_{-\infty}^{\infty} B_{j 1}\left(\mu_{k}\left(\eta_{1}\right) \sin \alpha-\eta_{1} \cos \alpha,-\mu_{k}\left(\eta_{1}\right) \cos \alpha-\eta_{1} \sin \alpha\right) \\
& \cdot \Lambda_{-}^{1 / 2-s}\left(\mu_{k}\left(\eta_{1}\right) \sin \alpha-\eta_{1} \cos \alpha,-\mu_{k}\left(\eta_{1}\right) \cos \alpha-\eta_{1} \sin \alpha\right) \\
& \cdot\left(\mu_{k}\left(\eta_{1}\right) \sin \alpha-\eta_{1} \cos \alpha-i 0\right)^{s-m_{j 1}-1 / 2} \\
& \cdot \widetilde{c_{k_{2}}}\left(\eta_{1}\right) e^{-i \mu_{k}\left(\eta_{1}\right) x_{1} \sin \alpha+i \eta_{1} x_{1} \cos \alpha} d \eta_{1} \\
&=h_{j 1}\left(x_{1}\right), \quad x_{1}>0,1 \leq j \leq m .
\end{aligned}
$$

We used in (2.30) that $\Lambda_{-}^{1 / 2-s}$ is a "minus"-operator and therefore the action of such an operator on $G_{k 1} c_{k 1}$ and $G_{k 2} c_{k 2}$ will be the same as that of a differential operator (see (3.12)-(3.15) in [5], where such computations were made).

\section{Denote}

$$
\begin{aligned}
& b_{j k 1}^{+}=B_{j 1}\left(1, \lambda_{k}\right)\left(\cos \frac{\alpha}{2}+\lambda_{k} \sin \frac{\alpha}{2}-i 0\right)^{1 / 2-s}, \\
& b_{j k 1}^{-}=B_{j 1}\left(-1,-\lambda_{k+m}\right)\left(-\cos \frac{\alpha}{2}-\lambda_{k+m} \sin \frac{\alpha}{2}-i 0\right)^{1 / 2-s} e^{-i \pi\left(s-m_{j 1}-1 / 2\right)} .
\end{aligned}
$$

It follows from $(2.20)$ that

$$
\begin{aligned}
& \mu_{k} \sin \alpha-\cos \alpha=\frac{\left(\sin \alpha-\lambda_{k} \cos \alpha\right) \sin \alpha}{-\cos \alpha-\lambda_{k} \sin \alpha}-\cos \alpha=\frac{1}{-\cos \alpha-\lambda_{k} \sin \alpha}, \\
& \quad-\mu_{k} \cos \alpha-\sin \alpha=\frac{\lambda_{k}}{-\cos \alpha-\lambda_{k} \sin \alpha}
\end{aligned}
$$

Since we assume here that $0<\alpha \leq \pi$ we have

$$
\begin{aligned}
\left(\frac{\cos \frac{\alpha}{2}+\lambda_{k} \sin \frac{\alpha}{2}}{-\cos \alpha-\lambda_{k} \sin \alpha}-i 0\right)^{1 / 2-s}\left(\frac{1}{-\cos \alpha-\lambda_{k} \sin \alpha}-i 0\right)^{s-1 / 2} & \\
& =\left(\cos \frac{\alpha}{2}+\lambda_{k} \sin \frac{\alpha}{2}-i 0\right)^{1 / 2-s}, \quad 1 \leq k \leq m
\end{aligned}
$$


Therefore we have using $(2.32),\left(2.32^{\prime}\right),(2.33)$

$$
\begin{aligned}
b_{j k 1}^{+}= & B_{j 1}\left(1, \lambda_{k}\right)\left(\cos \frac{\alpha}{2}+\lambda_{k} \sin \frac{\alpha}{2}-i 0\right)^{1 / 2-s} \\
= & B_{j 1}\left(\mu_{k} \sin \alpha-\cos \alpha,-\mu_{k} \cos \alpha-\sin \alpha\right) \\
& \cdot \Lambda_{-}^{1 / 2-s}\left(\mu_{k} \sin \alpha-\cos \alpha,-\mu_{k} \cos \alpha-\sin \alpha\right) \\
& \cdot\left(\mu_{k} \sin \alpha-\cos \alpha-i 0\right)^{s-m_{j 1}-1 / 2}
\end{aligned}
$$

Analogously

$$
\begin{aligned}
b_{j 1}^{-}= & B_{j 1}\left(-\left(\mu_{k+m} \sin \alpha-\cos \alpha\right), \mu_{k+m} \cos \alpha+\sin \alpha\right) \\
& \cdot \Lambda_{-}^{1 / 2-s}\left(-\left(\mu_{k+m} \sin \alpha-\cos \alpha\right), \mu_{k+m} \cos \alpha+\sin \alpha\right) \\
& \cdot\left(-\left(\mu_{k+m} \sin \alpha-\cos \alpha\right)-i 0\right)^{s-m_{j 1}-1 / 2} .
\end{aligned}
$$

Note that symbols in (2.30) are homogeneous of degree zero. Therefore using (2.34), (2.35) and computing integrals in $\xi_{1}$ and $\eta_{1}$ we obtain

$$
\begin{aligned}
\sum_{k=1}^{m} b_{j k_{1}}^{+} & \Pi_{+}^{-} c_{k_{1}}+\sum_{k=1}^{m} b_{j k_{1}}^{-} \Pi_{+}^{+} c_{k_{1}} \\
& +\sum_{k=1}^{m} b_{j k_{1}}^{+} \frac{1}{2 \pi i} \int_{-\infty}^{0} \frac{c_{k 2}(t) d t}{\mu_{k} x_{1} \sin \alpha-x_{1} \cos \alpha-t} \\
& -\sum_{k=1}^{m} b_{j k_{1}}^{-} \frac{1}{2 \pi i} \int_{-\infty}^{0} \frac{c_{k_{2}}(t) d t}{\mu_{k+m} x_{1} \sin \alpha-x_{1} \cos \alpha-t} \\
= & h_{j 1}\left(x_{1}\right), \quad 1 \leq j \leq m,
\end{aligned}
$$

where

$$
\begin{aligned}
\Pi_{+}^{ \pm} c_{k_{1}} & = \pm \frac{i}{2 \pi} \int_{0}^{\infty} \frac{c_{k_{1}}(t) d t}{x_{1}-t \pm i 0}=F^{-1} \theta\left(\mp \xi_{1}\right) \widetilde{c_{k_{1}}}\left(\xi_{1}\right), \\
\theta(t) & =1 \quad \text { for } t>0, \quad \theta(t)=0 \text { for } t<0 .
\end{aligned}
$$

It will be convenient to rewrite boundary conditions $(2.8)$ in coordinates $y=$ $\left(y_{1}, y_{2}\right)$ (see (2.9)). We have

$$
\left(\xi_{1} \cos \frac{\alpha}{2}+\xi_{2} \sin \frac{\alpha}{2}-i 0\right)^{1 / 2-s}=\left(-\eta_{1} \cos \frac{\alpha}{2}+\eta_{2} \sin \frac{\alpha}{2}-i 0\right)^{1 / 2-s},
$$

since $\xi=U_{-(\pi-\alpha)} \eta$. Therefore (2.8) will have the following form:

$$
\begin{gathered}
p_{2}^{-}\left(i \frac{\partial}{\partial y_{1}}+i 0\right)^{s-m_{j 2}-1 / 2} B_{j 2}^{(1)}\left(i \frac{\partial}{\partial y_{1}}, i \frac{\partial}{\partial y_{2}}\right) \\
\cdot\left(-i \frac{\partial}{\partial y_{1}} \cos \frac{\alpha}{2}+i \frac{\partial}{\partial y_{2}} \sin \frac{\alpha}{2}-i 0\right)^{1 / 2-s} l u \\
=h_{j 2}\left(y_{1}\right), \quad y_{1}<0,1 \leq j \leq m,
\end{gathered}
$$

where $B_{j 2}^{(1)}\left(\eta_{1}, \eta_{2}\right)$ is the symbol of $B_{j 2}$ in $\left(y_{1}, y_{2}\right)$ coordinates,

$$
B_{j 2}^{(1)}\left(\eta_{1}, \eta_{2}\right)=B_{j 2}\left(-\eta_{1} \cos \alpha+\eta_{2} \sin \alpha,-\eta_{1} \sin \alpha-\eta_{2} \cos \alpha\right) .
$$


Applying (2.8) to (2.15) and using (2.39) we obtain

$$
\begin{aligned}
\sum_{k=1}^{m} \frac{1}{2 \pi} \int_{-\infty}^{\infty} \int_{0}^{\infty} B_{j 2}\left(\xi_{1}, \lambda_{k}\left(\xi_{1}\right)\right) & \\
& \cdot \Lambda_{-}^{1 / 2-s}\left(\xi_{1}, \lambda_{k}\left(\xi_{1}\right)\right)\left(-\xi_{1} \cos \alpha-\lambda_{k}\left(\xi_{1}\right) \sin \alpha+i 0\right)^{s-m_{j 2}-1 / 2} \\
& \cdot c_{k 1}(t) e^{i \lambda_{k}\left(\xi_{1}\right) y_{1} \sin \alpha_{1}-i\left(-y_{1} \cos \alpha-t\right) \xi_{1}} d \xi_{1} d t \\
& +\sum_{k=1}^{m} \frac{1}{2 \pi} \int_{-\infty}^{\infty} B_{j 2}^{(1)}\left(\eta_{1}, \mu_{k}\left(\eta_{1}\right)\right)\left(-\eta_{1} \cos \frac{\alpha}{2}+\mu_{k}\left(\eta_{1}\right) \sin \frac{\alpha}{2}-i 0\right)^{1 / 2-s} \\
= & h_{j 2}\left(y_{1}\right), \quad y_{1}<0,1 \leq j \leq m .
\end{aligned}
$$

It follows from (2.41) analogously to (2.36) that

$$
\begin{aligned}
\sum_{k=1}^{m} b_{j k 2}^{+} \Pi_{-}^{-} c_{k 2} & +\sum_{k=1}^{m} b_{j k 2}^{-} \Pi_{-}^{+} c_{k 2} \\
& +\sum_{k-1}^{m} b_{j k 2}^{+} \frac{1}{2 \pi i} \int_{0}^{\infty} \frac{c_{k 1}(t) d t}{\left(-\lambda_{k} \sin \alpha-\cos \alpha\right) y_{1}-t} \\
& -\sum_{k=1}^{m} b_{j k 2}^{-} \frac{1}{2 \pi i} \int_{0}^{\infty} \frac{c_{k_{1}}(t) d t}{\left(-\lambda_{k+m} \sin \alpha-\cos \alpha\right) y_{1}-t} \\
= & h_{j 2}\left(y_{1}\right), \quad y_{1}<0,1 \leq j \leq m,
\end{aligned}
$$

where

$$
\begin{gathered}
\Pi_{-}^{ \pm} c_{k_{2}}= \pm \frac{i}{2 \pi} \int_{-\infty}^{0} \frac{c_{k_{2}}(t)}{y_{1}-t \pm i 0} d t \\
b_{j k 2}^{+}=B_{j 2}^{(1)}\left(1, \mu_{k}\right)\left(-\cos \frac{\alpha}{2}+\mu_{k} \sin \frac{\alpha}{2}-i 0\right)^{1 / 2-s}, \\
b_{j k 2}^{-}=B_{j 2}^{(1)}\left(-1,-\mu_{k+m}\right)\left(\cos \frac{\alpha}{2}-\mu_{k+m} \sin \frac{\alpha}{2}-i 0\right)^{1 / 2-s} \\
\cdot e^{i \pi\left(s-m_{j 2}-1 / 2\right)} .
\end{gathered}
$$

We used in (2.44) that (cf. (2.34), (2.35))

$$
\begin{aligned}
& B_{j 2}\left(1, \lambda_{k}\right) \Lambda_{-}^{1 / 2-s}\left(1, \lambda_{k}\right)\left(-\cos \alpha-\lambda_{k} \sin \alpha+i 0\right)^{s-m_{j 2}-1 / 2}=b_{j k_{2}}^{+}, \\
& B_{j 2}\left(-1,-\lambda_{k+m}\right) \Lambda_{-}^{1 / 2-s}\left(-1,-\lambda_{k+m}\right) \\
& \quad \cdot\left(\cos \alpha+\lambda_{k+m} \sin \alpha+i 0\right)^{s-m_{j 2}-1 / 2}=b_{j k_{2}}^{-}
\end{aligned}
$$

Changing $y_{1}$ to $-y_{1}$ and denoting

$$
c_{k_{2}}^{\prime}(t)=c_{k_{2}}(-t), \quad 0<t<+\infty,
$$


we obtain

$$
\begin{aligned}
\sum_{k=1}^{m} b_{j k_{2}}^{+} & \Pi_{+}^{+} c_{k_{2}}^{\prime}+\sum_{k=1}^{m} b_{j k_{2}}^{-} \Pi_{+}^{-} c_{k_{2}}^{\prime} \\
& +\sum_{k=1}^{m} b_{j k_{2}}^{+} \frac{1}{2 \pi i} \int_{0}^{\infty} \frac{c_{k_{1}}(t) d t}{\left(\lambda_{k} \sin \alpha+\cos \alpha\right) y_{1}-t} \\
& -\sum_{k=1}^{m} b_{j k_{2}}^{-} \frac{1}{2 \pi i} \int_{0}^{\infty} \frac{c_{k_{1}}(t) d t}{\left(\lambda_{k+m} \sin \alpha+\cos \alpha\right) y_{1}-t} \\
= & h_{j 2}\left(-y_{1}\right), \quad 0<y_{1}<+\infty, 1 \leq j \leq m .
\end{aligned}
$$

We used in (2.47) that

$$
\Pi_{-}^{ \pm}=\Pi_{+}^{\mp}
$$

after changing $y_{1}$ to $-y_{1}$ and $t$ to $-t$. Also, changing $t$ to $-t$ in (2.36) and using (2.32), we obtain

$$
\begin{aligned}
\sum_{k=1}^{m} b_{j k_{1}}^{+} & \Pi_{+}^{-} c_{k_{1}}+\sum_{k=1}^{m} b_{j k_{1}}^{-} \Pi_{+}^{+} c_{k_{1}} \\
& +\sum_{k=1}^{m} b_{j k_{1}}^{+} \frac{1}{2 \pi i} \int_{0}^{\infty} \frac{c_{k_{2}}^{\prime}(t) d t}{x_{1}\left(-\cos \alpha-\lambda_{k} \sin \alpha\right)^{-1}+t} \\
& -\sum_{k=1}^{m} b_{j k_{1}}^{-} \frac{1}{2 \pi i} \int_{0}^{\infty} \frac{c_{k_{2}}^{\prime}(t) d t}{x_{1}\left(-\cos \alpha-\lambda_{k+m} \sin \alpha\right)^{-1}+t} \\
= & h_{j 1}\left(x_{1}\right), \quad 1 \leq j \leq m, x_{1}>0 .
\end{aligned}
$$

Denote by $\hat{h}(z)$ or by $M(h)$ the Mellin transform of $h(t)$ :

$$
\hat{h}(z)=\int_{0}^{\infty} h(t) t^{z-1} d t .
$$

Applying the Mellin transform to

$$
\int_{0}^{\infty} \frac{c_{k 1}(t) d t}{d_{k} y_{1}-t}
$$

we obtain (see, for example, $[3, \S 15]$ )

$$
M\left(\int_{0}^{\infty} \frac{c_{k 1}(t)}{d_{k} y_{1}-t} d t\right)=2 \pi i \frac{e^{z \ln \left(d_{k}^{-1}\right)}}{1-e^{2 \pi i z}} \hat{c}_{k 1}(z),
$$

where $\ln \left(d_{k}^{-1}\right)=\ln \left|d_{k}^{-1}\right|+i \arg \left(d_{k}^{-1}\right), 0<\arg \left(d_{k}^{-1}\right)<2 \pi$. Note that (see [3, $\S 15])$

$$
\begin{aligned}
& M\left(\Pi_{+}^{+} c_{k 1}\right)=-\frac{e^{2 \pi i z}}{1-e^{2 \pi i z}} \hat{c}_{k 1}(z), \\
& M\left(\Pi_{+}^{-} c_{k 1}\right)=\frac{1}{1-e^{2 \pi i z}} \hat{c}_{k 1}(z)
\end{aligned}
$$


Taking into account (2.51), (2.52) and applying the Mellin transform to (2.49), (2.47), we obtain

$$
\begin{aligned}
& \sum_{k=1}^{m} b_{j k 1}^{+} \frac{\hat{c}_{k 1}(z)}{1-e^{2 \pi i z}}-\sum_{k=1}^{m} b_{j k 1}^{-} \frac{e^{2 \pi i z} \hat{c}_{k 1}(z)}{1-e^{2 \pi i z}} \\
&-\sum_{k=1}^{m} b_{j k 1}^{+} \frac{e^{i \beta_{k} z} \hat{c}_{k 2}^{\prime}(z)}{1-e^{2 \pi i z}}+\sum_{k=1}^{m} b_{j k 1}^{-} \frac{e^{i \beta_{k+m} z} \hat{c}_{k 2}^{\prime}(z)}{1-e^{2 \pi i z}} \\
&= \hat{h}_{j 1}(z), \quad 1 \leq j \leq m, z=\frac{1}{2}+i \tau,-\infty<\tau<+\infty \\
&-\sum_{k=1}^{m} b_{j k 2}^{+} \frac{e^{2 \pi i z} \hat{c}_{k 2}^{\prime}(z)}{1-e^{2 \pi i z}}+\sum_{k=1}^{m} b_{j k 2}^{-} \frac{\hat{c}_{k 2}^{\prime}(z)}{1-e^{2 \pi i z}} \\
& \quad+\sum_{k=1}^{m} b_{j k 2}^{+} \frac{e^{2 \pi i z-i \beta_{k} z} \hat{c}_{k 1}(z)}{1-e^{2 \pi i z}}-\sum_{k=1}^{m} b_{j k 2}^{-} \frac{e^{2 \pi i z-i \beta_{k+m} z} \hat{c}_{k 1}(z)}{1-e^{2 \pi i z}} \\
&=\hat{h}_{j 2}^{\prime}(z),
\end{aligned}
$$

where $1 \leq j \leq m, h_{j 2}^{\prime}\left(y_{1}\right)=h_{j 2}\left(-y_{1}\right), z=\frac{1}{2}+i \tau, \tau \in \mathbf{R}$,

$$
\begin{gathered}
i \beta_{k}=\ln \left(\cos \alpha+\lambda_{k} \sin \alpha\right)=\ln \left|\cos \alpha+\lambda_{k} \sin \alpha\right| \\
+i \arg \left(\cos \alpha+\lambda_{k} \sin \alpha\right), \quad 1 \leq k \leq 2 m, \\
0<\arg \left(\cos \alpha+\lambda_{k} \sin \alpha\right)<2 \pi
\end{gathered}
$$

Denote

$$
b_{i}^{ \pm}=\left\|b_{j k i}^{ \pm}\right\|_{j, k=1}^{m}, \quad b_{i}=\left(b_{i}^{+}\right)^{-1} b_{i}^{-} .
$$

We assume that the Shapiro-Lopatinsky condition is satisfied, i.e.,

$$
\operatorname{det} b_{1}^{+} \neq 0, \quad \operatorname{det} b_{2}^{-} \neq 0 \text {. }
$$

Multiplying (2.53) by $\left(b_{1}^{+}\right)^{-1}$ and $\left(2.53^{\prime}\right)$ by $\left(b_{2}^{+}\right)^{-1}$ we obtain

$$
\begin{aligned}
& \left(I-b_{1} e^{2 \pi i z}\right)\left(1-e^{2 \pi i z}\right)^{-1} \hat{c}_{1}-\left(\Delta_{1}(z)-b_{1} \Delta_{2}(z)\right) \\
& \quad \cdot\left(1-e^{2 \pi i z}\right)^{-1} \hat{c}_{2}=\hat{h}_{1}, \\
& \left(-e^{2 \pi i z} I-b_{2}\right)\left(1-e^{2 \pi i z}\right)^{-1} \hat{c}_{2}+\left(e^{2 \pi i z} \Delta_{1}^{-1}(z)-b_{2} e^{2 \pi i z} \Delta_{2}^{-1}(z)\right) \\
& \quad \cdot\left(1-e^{2 \pi i z}\right)^{-1} \hat{c}_{1}=\hat{h}_{2}(z),
\end{aligned}
$$

where

$$
\begin{aligned}
& \hat{c}_{1}=\left(\hat{c}_{11}, \ldots, \hat{c}_{m 1}\right), \quad \hat{c}_{2}=\left(\hat{c}_{12}^{\prime}, \ldots, \hat{c}_{m 2}^{\prime}\right), \\
& \hat{h}_{1}=\left(b_{1}^{+}\right)^{-1}\left(\hat{h}_{11}, \ldots, \hat{h}_{m 1}\right), \quad \hat{h}_{2}=\left(b_{2}^{+}\right)^{-1}\left(\hat{h}_{12}^{\prime}, \ldots, \hat{h}_{m 2}^{\prime}\right),
\end{aligned}
$$

$\Delta_{1}(z), \Delta_{2}(z)$ are diagonal matrices

$$
\Delta_{1}(z)=\left\|e^{i \beta_{k} z} \delta_{j k}\right\|_{j, k=1}^{m}, \quad \Delta_{2}(z)=\left\|e^{i \beta_{k+m} z} \delta_{j k}\right\|_{j, k=1}^{m} .
$$


Taking into account the Parseval formula for the Mellin transform

$$
\int_{0}^{\infty}|h(t)|^{2} d t=\frac{1}{2 \pi i} \int_{1 / 2-i \infty}^{1 / 2+i \infty}|\hat{h}(z)|^{2} d z
$$

and the relation

$$
M(t d h / d t)=-z \hat{h}(z),
$$

we obtain that

$$
\operatorname{det} M(z) \neq 0, \quad z=\frac{1}{2}+i \tau, \tau \in \mathbf{R},
$$

is a necessary and sufficient condition for the invertibility in $\mathscr{H}_{0, N_{1}}, \forall N_{1} \geq 0$, of the operator $\Phi$ defined by the left-hand sides of (2.36), (2.42). Here $M(z)$ is the matrix of the system (2.57). Note that $R_{0} \Phi^{-1}$ will be the right inverse to $\mathscr{A}$ :

$$
\mathscr{A} R_{0} \Phi^{-1}=I .
$$

We shall now simplify condition (2.61). Denote

$$
\begin{aligned}
& \left(1-e^{2 \pi i z}\right)^{-1} \hat{c}_{1}-\left(1-e^{2 \pi i z}\right)^{-1} \Delta_{1}(z) \hat{c}_{2}=\hat{d}_{1}(z), \\
& \left(1-e^{2 \pi i z}\right)^{-1} e^{2 \pi i z} \Delta_{2}^{-1}(z) \hat{c}_{1}-\left(1-e^{2 \pi i z}\right)^{-1} \hat{c}_{2}=\hat{d}_{2}(z) .
\end{aligned}
$$

Let $\Delta_{3}(z)$ be the matrix of the system (2.62). Since $\Delta_{1}(z), \Delta_{2}(z)$ are diagonal we obtain

$$
\begin{aligned}
\operatorname{det} \Delta_{3}(z) & =\left(1-e^{2 \pi i z}\right)^{-1} \prod_{k=1}^{m} \operatorname{det} \| \begin{array}{cc}
1 & -e^{i \beta_{k} z}
\end{array} \mid \\
& =\left(1-e^{2 \pi i z-i \beta_{k+m}}\right)^{-1} \prod_{k=1}^{m}\left(-1+e^{i\left(2 \pi-\beta_{k+m}+\beta_{k}\right) z}\right) .
\end{aligned}
$$

We shall assume that

$$
-1+e^{i\left(2 \pi-\beta_{k+m}+\beta_{k}\right) z} \neq 0, \quad z=\frac{1}{2}+i \tau, \quad \forall \tau \in \mathbf{R}, 1 \leq k \leq m .
$$

The case when (2.64) is not satisfied will be considered in Remark 2.2.

When (2.64) holds, system (2.62) is invertible for any $z=\frac{1}{2}+i \tau$ and system (2.57) is equivalent to the following system:

$$
\begin{gathered}
\hat{d}_{1}-b_{1} \Delta_{2} \hat{d}_{2}=\hat{h}_{1}, \\
-b_{2} \hat{d}_{2}+e^{2 \pi i z} \Delta_{1}^{-1} \hat{d}_{1}=\hat{h}_{2} .
\end{gathered}
$$

Substituting $\hat{d}_{1}$ from (2.65) into $\left(2.65^{\prime}\right)$ we obtain

$$
\left(-b_{2}+e^{2 \pi i z} \Delta_{1}^{-1} b_{1} \Delta_{2}\right) \hat{d}_{2}=\hat{h}_{2}-e^{2 \pi i z} \Delta_{1}^{-1} \hat{h}_{1} .
$$

Therefore the condition (2.61) is satisfied iff (2.64) and the following condition hold:

$$
\operatorname{det}\left(-b_{2}+e^{2 \pi i z} \Delta_{1}^{-1}(z) b_{1} \Delta_{2}(z)\right) \neq 0, \quad z=\frac{1}{2}+i \tau, \quad \forall \tau \in \mathbf{R} .
$$


We shall find more explicitly the dependence of $b_{1}$ and $b_{2}$ on $s$. It follows from (2.31), (2.44) that

$$
\begin{aligned}
b_{j k 1}= & \left(\cos \frac{\alpha}{2}+\lambda_{j} \sin \frac{\alpha}{2}-i 0\right)^{s-1 / 2} \\
& \cdot b_{j k 1}^{(0)}\left(-\cos \frac{\alpha}{2}-\lambda_{k+m} \sin \frac{\alpha}{2}-i 0\right)^{1 / 2-s} e^{-i \pi(s-1 / 2)}, \\
b_{j k 2}= & \left(-\cos \frac{\alpha}{2}+\mu_{j} \sin \frac{\alpha}{2}-i 0\right)^{s-1 / 2} \\
& \cdot b_{j k 2}^{(0)}\left(\cos \frac{\alpha}{2}-\mu_{k+m} \sin \frac{\alpha}{2}-i 0\right)^{1 / 2-s} e^{i \pi(s-1 / 2)},
\end{aligned}
$$

where matrices $b_{r}^{(0)}=\left\|b_{j k r}^{(0)}\right\|_{j, k=1}^{m}, r=1,2$, are independent of $s$,

$$
\begin{aligned}
& b_{r}^{(0)}=\left(B_{r}^{+}\right)^{-1}\left\|e^{i \pi m_{j r}} \delta_{j k}\right\|_{j, k=1}^{m} B_{r}^{-}, \quad r=1,2, \\
& B_{1}^{+}=\left\|B_{j 1}\left(1, \lambda_{k}\right)\right\|, \quad B_{1}^{-}=\left\|B_{j 1}\left(-1,-\lambda_{k+m}\right)\right\|, \\
& B_{2}^{+}=\left\|B_{j 2}^{(1)}\left(1, \mu_{k}\right)\right\|, \quad B_{2}^{-}=\left\|B_{j 2}^{(1)}\left(-1,-\mu_{k+m}\right)\right\| .
\end{aligned}
$$

It follows from (2.20) that

$$
\begin{aligned}
\left(-\cos \frac{\alpha}{2}+\mu_{k} \sin \frac{\alpha}{2}-i 0\right)^{s-1 / 2}=\left(\frac{\cos (\alpha / 2)+\lambda_{k} \sin (\alpha / 2)}{-\cos \alpha-\lambda_{k} \sin \alpha}-i 0\right)^{s-1 / 2} \\
=\left(\cos \frac{\alpha}{2}+\lambda_{k} \sin \frac{\alpha}{2}-i 0\right)^{s-1 / 2}\left(-\cos \alpha-\lambda_{k} \sin \alpha+i 0\right)^{1 / 2-s} \\
=\left(\cos \frac{\alpha}{2}+\lambda_{k} \sin \frac{\alpha}{2}-i 0\right)^{s-1 / 2} e^{i\left(\beta_{k}-\pi\right)(1 / 2-s)}
\end{aligned}
$$

and analogously

$$
\begin{aligned}
\left(\cos \frac{\alpha}{2}-\mu_{k+m}\right. & \left.\sin \frac{\alpha}{2}-i 0\right)^{1 / 2-s} \\
& =\left(-\cos \frac{\alpha}{2}-\lambda_{k+m} \sin \frac{\alpha}{2}-i 0\right)^{1 / 2-s} e^{i\left(\beta_{k+m}-\pi\right)(s-1 / 2)} .
\end{aligned}
$$

Multiplying the matrix in (2.67) by

$$
\left\|\left(-\cos \frac{\alpha}{2}+\mu_{k} \sin \frac{\alpha}{2}-i 0\right)^{s-1 / 2} \delta_{j k}\right\|^{-1}
$$

from the left and by

$$
\left\|\left(\cos \frac{\alpha}{2}-\mu_{k+m} \sin \frac{\alpha}{2}-i 0\right)^{1 / 2-s} e^{-i \pi(s-1 / 2)} \delta_{j k}\right\|
$$

from the right, we obtain using (2.71) and (2.72) that the condition (2.67) is equivalent to the following condition:

$$
\operatorname{det} M_{0}\left(z-s+\frac{1}{2}\right) \neq 0, \quad \forall z=\frac{1}{2}+i \tau,
$$

where

$$
M_{0}(z)=-b_{2}^{(0)}+e^{2 \pi i z} \Delta_{1}^{-1}(z) b_{1}^{(0)} \Delta_{2}(z) .
$$


Function $\operatorname{det} M_{0}(z)$ is an entire analytic function of exponential type having infinitely many zeros:

$$
\operatorname{det} M_{0}\left(z_{k}\right)=0, \quad k=1,2, \ldots
$$

Let $\Sigma$ be the set of all $s \in \mathbf{R}^{1}$ such that (2.73) is not satisfied. Then

$$
s \in \Sigma \text { iff } s=1-\operatorname{Re} z_{k} \text { for some } k, \text { where } \operatorname{det} M_{0}\left(z_{k}\right)=0 .
$$

Remark 2.1. Until now we have assumed that $0<\alpha \leq \pi$. Now consider the case when $\pi<\alpha<2 \pi$. Note that $G_{j 1}\left(x_{2}, x_{1}-t\right)$ (see (2.23)) is well defined and real analytic for all $\left(x_{1}, x_{2}\right) \in \mathbf{R}^{2} \backslash \Gamma_{1}$. Analogously $G_{j 2}\left(y_{2}, y_{1}-t\right)$ (see (2.24)) is well defined for $\left(y_{1}, y_{2}\right) \in \mathbf{R}^{2} \backslash \Gamma_{2}$. Therefore $u\left(x_{1}, x_{2}\right)$ is defined by (2.25) for any angle $\alpha, 0<\alpha<2 \pi$. We can consider (2.25) as an analytic extension of (2.15) that is defined only when $x_{2}>0$ and $y_{2}>0$. The Fourier transform of $G_{j 1}\left(x_{2}, x_{1}\right)$ has the form

$$
\widetilde{G_{j 1}}\left(\xi_{1}, \xi_{2}\right)=\frac{i}{\xi_{2}-\lambda_{j} \xi_{1}}+\frac{i}{\xi_{2}-\lambda_{j+m} \xi_{1}} .
$$

Analogously

$$
\widetilde{G_{j 2}}\left(\eta_{1}, \eta_{2}\right)=\frac{i}{\eta_{2}-\mu_{j} \eta_{1}}+\frac{i}{\eta_{2}-\mu_{j+m} \eta_{1}} .
$$

The application of boundary conditions (2.8) to $G_{k 2} c_{k 2}$ is the same as in the case when $0<\alpha \leq \pi$. Now apply $B_{j 2} \Lambda_{-}^{1 / 2-s}$ to $G_{k 1} c_{k 1}$ using (2.77) and making change of coordinates $y=U_{\pi-\alpha} x, \eta=U_{\pi-\alpha} \xi$. We obtain

$$
\begin{array}{r}
p_{\Omega} B_{j 2} \Lambda_{-}^{1 / 2-s} G_{k 1} c_{k 1} \\
=\frac{1}{(2 \pi)^{2}} \int_{0}^{\infty} \int_{\mathbf{R}^{2}} B_{j 2}^{(1)}\left(\eta_{1}, \eta_{2}\right)\left(-\eta_{1} \cos \frac{\alpha}{2}+\eta_{2} \sin \frac{\alpha}{2}-i 0\right)^{1 / 2-s} \\
\cdot i\left(\frac{\mu_{k} \sin \alpha-\cos \alpha}{\eta_{2}-\mu_{k} \eta_{1}}+\frac{\mu_{k+m} \sin \alpha-\cos \alpha}{\eta_{2}-\mu_{k+m} \eta_{1}}\right) \\
\cdot e^{-i y_{1} \eta_{1}-i y_{2} \eta_{2}+i t\left(-\eta_{1} \cos \alpha+\eta_{2} \sin \alpha\right)} c_{k 1}(t) d t d \eta_{1} d \eta_{2} .
\end{array}
$$

Now taking the restriction to $\Gamma_{2}=\left\{y_{2}=0, y_{1}<0\right\}$, computing the integral in $\eta_{2}$ using the Jordan lemma, and applying

$$
p_{\Gamma_{2}}\left(i \partial / \partial y_{1}+i 0\right)^{s-m_{j 2}-1 / 2},
$$

we obtain (2.42) where $b_{j k 2}^{ \pm}$have the form (2.44). Analogously (2.36) holds for $\pi<\alpha<2 \pi$ where $b_{j k 1}^{ \pm}$are given by (2.31). Note a modification of the proof that $u \in H_{1 / 2, N_{1}}^{(1)}(G)$ (see (2.26)-(2.29)). Let $E_{2}\left(\xi_{1}, \xi_{2}\right)$ be the same "minus"-symbol as in (3.10) of $\S 3$. Let constant $c_{0}$ be such that $\widetilde{G}_{j 1}(0,1)-$ $c_{0} E_{2}(0,1)=0$. Then $\left(\widetilde{G}_{j 1}\left(\xi_{1}, \xi_{2}\right)-c_{0} E_{2}\left(\xi_{1}, \xi_{2}\right)\right) \widetilde{c_{j 1}}\left(\xi_{1}\right)$ satisfies $(2.28)$ and 
$p_{G} G_{j 1} c_{j 1}=p_{G}\left(G_{j 1} c_{j 1}-c_{0} E_{2} c_{j 1}\right)$ since $p_{G} E_{2} c_{j 1}=0$. Therefore all previous results of this section hold without any change for $\pi<\alpha<2 \pi$. Note that the case $\alpha=2 \pi$ can be treated by methods of [3] (see Remark 24.2 in [3]).

Remark 2.2. It follows from (2.62), (2.65), $\left(2.65^{\prime}\right)$, (2.66) that

$$
\operatorname{det} M(z)=\operatorname{det} \Delta_{3} \operatorname{det}\left(-b_{2}+e^{2 \pi i z} \Delta_{1}^{-1} b_{1} \Delta_{2}\right) .
$$

Therefore if the condition (2.64) is not satisfied then (2.61) fails for any $s \in \mathbf{R}^{1}$. Note that (2.64) is always satisfied when $A_{0}\left(\xi_{1}, \xi_{2}\right)$ has real coefficients. Indeed in this case one can choose

$$
\lambda_{j+m}=\bar{\lambda}_{j}, \quad 1 \leq j \leq m .
$$

Then (see (2.54))

$$
\beta_{j+m}=2 \pi-\bar{\beta}_{j}
$$

and therefore

$$
2 \pi+\beta_{j}-\beta_{j+m}=2 \operatorname{Re} \beta_{j}, \quad 0<\operatorname{Re} \beta_{j}<2 \pi,
$$

so that $(2.64)$ is satisfied. Note when $(2.64)$ is not satisfied it means that the Ansatz (2.15) does not work. In this case we shall replace (2.15) by a more general Ansatz,

$$
\begin{aligned}
u= & \sum_{j, p=1}^{m} \frac{1}{2 \pi} \int_{-\infty}^{\infty} e^{-x_{2} \lambda_{j}\left(\xi_{1}\right)-i x_{1} \xi_{1}} d_{j p 1}\left(\xi_{1}\right) \widetilde{c_{p 1}}\left(\xi_{1}\right) d \xi_{1} \\
& +\sum_{j, p=1}^{m} \frac{1}{2 \pi} \int_{-\infty}^{\infty} e^{-i y_{2} \mu_{j}\left(\eta_{1}\right)-i y_{1} \eta_{1}} d_{j p 2}\left(\eta_{1}\right) \widetilde{c_{p 2}}\left(\eta_{1}\right) d \eta_{1}
\end{aligned}
$$

where

$$
\begin{aligned}
& c_{p 1}=0 \text { for } x_{1}<0, \quad c_{p 2}=0 \text { for } y_{1}>0, \\
& d_{j p i}\left(\xi_{1}\right)=d_{j p i}^{+} \theta\left(\xi_{1}\right)+d_{j p i}^{-} \theta\left(-\xi_{1}\right) .
\end{aligned}
$$

Then instead of (2.57) we obtain

$$
\begin{aligned}
& \left(D_{1}^{+}-b_{1} e^{2 \pi i z} D_{1}^{-}\right)\left(1-e^{2 \pi i z}\right)^{-1} \hat{c}_{1}-\left(\Delta_{1}(z) D_{2}^{+}-b_{1} \Delta_{2}(z) D_{2}^{-}\right) \\
& \quad \cdot\left(1-e^{2 \pi i z}\right)^{-1} \hat{c}_{2}=\hat{h}_{1}, \\
& \left(-e^{2 \pi i z} D_{2}^{+}-b_{2} D_{2}^{-}\right)\left(1-e^{2 \pi i z}\right)^{-1} \hat{c}_{2}+\left(e^{2 \pi i z} \Delta_{1}^{-1}(z) D_{1}^{+}-b_{2} e^{2 \pi i z} \Delta_{2}^{-1}(z) D_{1}^{-}\right) \\
& \quad \cdot\left(1-e^{2 \pi i z}\right)^{-1} \hat{c}_{1}=\hat{h}_{2},
\end{aligned}
$$

where

$$
D_{i}^{ \pm}=\left\|d_{j p i}^{ \pm}\right\|_{j, p=1}^{m}, \quad i=1,2 .
$$

Denote analogously to (2.62):

$$
\begin{aligned}
& \left(D_{1}^{+} \hat{c}_{1}-\Delta_{1}(z) D_{2}^{+} \hat{c}_{2}\right)\left(1-e^{2 \pi i z}\right)^{-1}=\hat{d}_{1}(z), \\
& e^{2 \pi i z} \Delta_{2}^{-1}(z)\left(1-e^{2 \pi i z}\right)^{-1} D_{1}^{-} \hat{c}_{1}-\left(1-e^{2 \pi i z}\right)^{-1} D_{2}^{-} \hat{c}_{2}=\hat{d}_{2}(z) .
\end{aligned}
$$


Then $\hat{d}_{1}, \hat{d}_{2}$ satisfy $(2.65),\left(2.65^{\prime}\right)$. Therefore

$$
\operatorname{det} M^{\prime}(z)=\operatorname{det}\left(-b_{2}+e^{2 \pi i z} \Delta_{1}^{-1} b_{1} \Delta_{2}\right) \operatorname{det} \Delta_{4},
$$

where

$$
\operatorname{det} \Delta_{4}=\operatorname{det}\left\|\begin{array}{cc}
D_{1}^{+} & -\Delta_{1}(z) D_{2}^{+} \\
e^{2 \pi i z} \Delta_{2}^{-1} D_{1}^{-} & -D_{2}^{-}
\end{array}\right\| \text {. }
$$

It is clear that choosing $D_{1}^{ \pm} ; D_{2}^{ \pm}$one can achieve that

$$
\operatorname{det} \Delta_{4} \neq 0 \text { for } z=\frac{1}{2}+i \tau, \tau \in \mathbf{R} \text {. }
$$

Therefore the condition (2.73) is the only condition for the existence of a solution of boundary value problem (2.1), (2.7), (2.8).

Example 2.1. Consider the case when $\alpha=\pi$. Then $\beta_{k}=\pi, 1 \leq k \leq 2 m$, and the condition (2.64) is satisfied. Also $\Delta_{1}(z)=\Delta_{2}(z)=e^{\pi i z} I$ where $I$ is the identity matrix. The condition (2.73) is equivalent to the condition

$$
\operatorname{det}\left(-e^{2 \pi \tau} I+e^{-2 \pi i s}\left(b_{2}^{(0)}\right)^{-1} b_{1}^{(0)}\right) \neq 0, \quad \forall \tau \in \mathbf{R}^{1} .
$$

Let $a_{j}$ be the eigenvalues of

$$
\left(b_{2}^{(0)}\right)^{-1} b_{1}^{(0)}, \quad 1 \leq j \leq m \quad \text { and } \quad \gamma_{j}=\frac{1}{2 \pi i} \ln a_{j}, \quad-\frac{1}{2}<\operatorname{Re} \gamma_{j} \leq \frac{1}{2} .
$$

Then (2.90) is equivalent to the condition

$$
s-\operatorname{Re} \gamma_{j} \neq 0 \quad(\bmod k), \quad 1 \leq j \leq m, k \in \mathbf{Z} .
$$

Note that roots of $\operatorname{det} M_{0}(z)$ have the following form in the case when $\alpha=\pi$ :

$$
z_{k j}=-\gamma_{j}+k, \quad 1 \leq j \leq m, k \in \mathbf{Z} .
$$

Replacing $s$ by $s+\frac{1}{2}$ we find that (2.91) is equivalent to corresponding condition in $[3, \S 14]$.

Example 2.2. Consider the case when $A_{0}\left(i \partial / \partial x_{1}, i \partial / \partial x_{2}\right)$ is the Laplacian $\partial^{2} / \partial x_{1}^{2}+\partial^{2} / \partial x_{2}^{2}$. We have $\lambda_{1}=-i, \lambda_{2}=i, \beta_{1}=2 \pi-\alpha, \beta_{2}=\alpha$. Therefore $M_{0}\left(z_{k}\right)=0$ iff

that is,

$$
e^{-2 \pi i z_{k}} e^{i(2 \pi-\alpha) z_{k}} e^{-i \alpha z_{k}}=b_{1}^{(0)} / b_{2}^{(0)},
$$

where $-\pi<\operatorname{Im} \ln \left(b_{2}^{(0)}\right)^{-1} b_{1}^{(0)} \leq \pi, k \in \mathbf{Z}$.

Therefore $s \in \Sigma$ iff

$$
s=1+\operatorname{Re}\left(\frac{1}{2 i \alpha} \ln \left(b_{2}^{(0)}\right)^{-1} b_{1}^{(0)}\right)+\frac{\pi k}{\alpha}
$$

for some $k \in \mathbf{Z}$. Here

$$
b_{1}^{(0)}=\frac{e^{i \pi m_{1}} B_{1}(-1,-i)}{B_{1}(1,-i)}, \quad b_{2}^{(0)}=\frac{e^{i \pi m_{2}} B_{2}^{(1)}(-1,-i)}{B_{2}^{(1)}(1,-i)} .
$$


Using (2.40) one can check that (2.94) coincides with condition (3.37) in [5] since in $[5,(3.37)]$ we had $B_{1}(1,-i)=1, B_{2}(1,-i)=e^{i(\pi-\alpha) m_{2}}$.

\section{UNIQUENESS OF THE SOLUTION OF THE MODEL PROBLEM}

In this section we shall prove:

Theorem 3.1. Any solution of (2.1) belonging to $H_{1 / 2, N_{1}}^{(1)}(G), N_{1} \geq 0$, can be represented in the form (2.15) with $\left(c_{1}, c_{2}\right) \in \mathscr{H}_{0, N_{1}}$, that is, $\operatorname{Im} R_{0}=\operatorname{ker} A_{0} \cap$ $H_{1 / 2, N_{1}}^{(1)}(G)$. (We assume that (2.64) is satisfied. Otherwise see Remark 2.2.)

Consider some boundary value problem $(2.1),(2.7),(2.8)$ that satisfies conditions (2.56) and (2.61). Then the fact that $\operatorname{Im} R_{0}=\operatorname{ker} A_{0} \cap H_{1 / 2, N_{1}}^{(1)}(G)$ is equivalent to the uniqueness of the solution of the boundary value problem (2.1), (2.7), (2.8) in $H_{1 / 2, N_{1}}^{(1)}(G)$. Indeed the uniqueness implies that there is one-to-one correspondence between $\operatorname{ker} A_{0} \cap H_{1 / 2, N_{1}}^{(1)}(G)$ and the right-hand sides $F \in \mathscr{H}_{0, N_{1}}$ of the boundary conditions $(2.7),(2.8)$. Then $u$ is equal to $R_{0} \Phi^{-1} F$, where $\Phi$ is the operator defined by equation (2.36), (2.42), since $\mathscr{A} u=F$ and $\mathscr{A} R_{0} \Phi^{-1} F=F$.

Proof of Theorem 3.1. Let $u \in H_{1 / 2, N_{1}}^{(1)}(G)$ be a solution of (2.1) satisfying the following boundary conditions:

$$
\begin{aligned}
& p_{1}^{+}\left(i \frac{\partial}{\partial x_{1}}-i 0\right)^{-m_{j 1}} B_{j 1}^{(0)}\left(i \frac{\partial}{\partial x_{1}}, i \frac{\partial}{\partial x_{2}}\right) l u=0, \\
& p_{2}^{-}\left(i \frac{\partial}{\partial y_{1}}+i 0\right)^{-m_{j 2}} B_{j 2}^{(0)}\left(i \frac{\partial}{\partial x_{1}}, i \frac{\partial}{\partial x_{2}}\right) l u=0,
\end{aligned}
$$

where $B_{j i}^{(0)}$ are such that (2.56) and (2.61) are satisfied for the boundary value problem (2.1), (3.1), (3.2). Note that we took $s=\frac{1}{2}$ in (2.7), (2.8). Since

$$
\left(i \frac{\partial}{\partial x_{1}}-i 0\right)^{-m_{j 1}} \text { and }\left(i \frac{\partial}{\partial y_{1}}+i 0\right)^{-m_{j 2}}
$$

are "minus"-operators with respect to $\Gamma_{1}$ and $\Gamma_{2}$, respectively, we have that

$$
\begin{array}{cc}
A_{0}\left(i \frac{\partial}{\partial x_{1}}, i \frac{\partial}{\partial x_{2}}\right) u=0 & \text { in } G, \\
B_{j 1}^{(0)}\left(i \frac{\partial}{\partial x_{1}}, i \frac{\partial}{\partial x_{2}}\right) u=0 & \text { on } \Gamma_{1}, \\
B_{j 2}^{(0)}\left(i \frac{\partial}{\partial x_{1}}, i \frac{\partial}{\partial x_{2}}\right) u=0 & \text { on } \Gamma_{2} .
\end{array}
$$

We shall assume that $B_{j 1}^{(0)}\left(\xi_{1}, \xi_{2}\right)$ are homogeneous polynomials, $\operatorname{deg} B_{j i}^{(0)} \leq$ $2 m-1, i=1,2,1 \leq j \leq m, N_{1} \geq 2 m$. 
Introducing polar coordinates $(r, \varphi)$ in $G$ and multiplying (3.3) by $r^{2 m}$, (3.4) by $r^{m_{j 1}}$, and (3.5) by $r^{m_{j 2}}$, we obtain

$$
A_{0}^{(1)}\left(\varphi, r \frac{\partial}{\partial r}, \frac{\partial}{\partial \varphi}\right) v(r, \varphi)=0,
$$

where $0<r<+\infty, 0<\varphi<\alpha, v(r, \varphi)=u\left(x_{1}, x_{2}\right)$,

$$
\begin{aligned}
& \left.B_{j 1}^{(1)}\left(\varphi, r \frac{\partial}{\partial r}, \frac{\partial}{\partial \varphi}\right) v(r, \varphi)\right|_{\varphi=0}=0, \\
& \left.B_{j 2}^{(1)}\left(\varphi, r \frac{\partial}{\partial r}, \frac{\partial}{\partial \varphi}\right) v(r, \varphi)\right|_{\varphi=\alpha}=0 .
\end{aligned}
$$

Here

$$
\begin{aligned}
& A_{0}^{(1)}\left(\varphi, r \frac{\partial}{\partial r}, \frac{\partial}{\partial \varphi}\right)=\sum_{k=0}^{2 m} a_{k}\left(\varphi, \frac{\partial}{\partial \varphi}\right)\left(r \frac{\partial}{\partial r}\right)^{k} \\
& B_{j k}^{(1)}\left(\varphi, r \frac{\partial}{\partial r}, \frac{\partial}{\partial \varphi}\right)=\sum_{p=0}^{m_{j k}} b_{j k p}\left(\varphi, \frac{\partial}{\partial \varphi}\right)\left(r \frac{\partial}{\partial r}\right)^{p},
\end{aligned}
$$

where

$$
a_{k}(\varphi, \partial / \partial \varphi), \quad b_{j k p}(\varphi, \partial / \partial \varphi)
$$

are differential operators in $\varphi$ with periodic coefficients, $\operatorname{deg} a_{k} \leq 2 m-k$, $\operatorname{deg} b_{j k p} \leq m_{j k}-p$. Note that

$$
\left[\left|\xi_{1}\right|^{s-1 / 2} F_{x_{1}} u\left(x_{1}, 0\right)\right]_{0} \leq C\left\|\Lambda_{0}^{s} u\left(x_{1}, x_{2}\right)\right\|_{0} \text { for } s>\frac{1}{2} .
$$

Since

$$
u\left(x_{1}, x_{2}\right) \in H_{1 / 2, N_{1}}^{(1)}(G), \quad N_{1} \geq 2 m,
$$

we have that for any $\theta, 0 \leq \theta \leq \alpha$, the restriction of $(r(\partial / \partial r))^{k}\left(\partial^{p} v / \partial \varphi^{p}\right)$ to the ray $\Gamma_{\theta}=\{\varphi=\theta, 0<r<+\infty\}$ exists and belongs to $L_{2}\left(\Gamma_{\theta}\right)$ where $0 \leq k+p \leq 2 m, p \leq 2 m-1$ : Denote by $\hat{v}(z, \varphi)$ the Mellin transform of $v(r, \varphi)$ :

$$
\hat{v}(z, \varphi)=\int_{0}^{\infty} v(r, \varphi) r^{z-1} d r .
$$

Using (2.60) and the Parseval formula (2.59) we obtain

$$
\begin{aligned}
\int_{0}^{\infty}\left|r \frac{\partial}{\partial r} v(r, \theta)\right|^{2} d r & =\frac{1}{2 \pi i} \int_{1 / 2-i \infty}^{1 / 2+i \infty}|z \hat{v}(z, \theta)|^{2} d z \\
& \geq \frac{C}{2 \pi i} \int_{1 / 2-i \infty}^{1 / 2+i \infty}|\hat{v}(z, \theta)|^{2} d z=C_{1} \int_{0}^{\infty}|v(r, \theta)|^{2} d r .
\end{aligned}
$$

Note that one should use (3.12) with $v \in C_{0}^{\infty}\left(\mathbf{R}^{2}\right)$ and then take the closure in $H_{1 / 2, N_{1}}^{(1)}(G)$. An analogous estimate holds for $\partial / \partial r(r v)$. It follows from (3.10), 
(3.12) that $v(r, \theta) \in L_{2}\left(\Gamma_{\theta}\right)$ for each $\theta$ and $\partial^{k} v(r, \theta) / \partial \varphi^{k} \in H_{0, N_{1}-k}\left(\Gamma_{\theta}\right)$, $0 \leq k \leq 2 m-1$.

Therefore performing the Mellin transform with respect to $r$ in (3.6), (3.7), (3.8) we obtain

$$
\begin{gathered}
A_{0}^{(1)}\left(\varphi,-z, \frac{\partial}{\partial \varphi}\right) \hat{v}(z, \varphi)=0, \\
\left.B_{j 1}^{(1)}\left(\varphi,-z, \frac{\partial}{\partial \varphi}\right) \hat{v}(z, \varphi)\right|_{\varphi=0}=0, \\
\left.B_{j 2}^{(1)}\left(\varphi,-z, \frac{\partial}{\partial \varphi}\right) \hat{v}(z, \varphi)\right|_{\varphi=\alpha}=0 .
\end{gathered}
$$

An arbitrary solution of (3.13) has the following form:

$$
\hat{v}(z, \varphi)=\sum_{k=1}^{2 m} \frac{\partial^{k-1} \hat{v}(z, 0)}{\partial \varphi^{k-1}} v_{k}(z, \varphi),
$$

where $v_{k}(z, \varphi), 1 \leq k \leq 2 m$, is the fundamental system of the ordinary differential equation (3.13), $v_{k}(z, \varphi)$ are entire functions of $z$, and

$$
\partial^{p-1} \hat{v}_{k}(z, 0) / \partial \varphi^{p-1}=\delta_{k p} .
$$

Note that $\partial^{k-1} \hat{v}(z, 0) / \partial \varphi^{k-1} \in L_{2}\left(\mathbf{R}^{1}\right)$ for $z=\frac{1}{2}+i \tau$. Substituting (3.16) into (3.14), (3.15) we obtain a $2 m \times 2 m$ homogeneous system for

$$
\begin{array}{cc}
\partial^{k-1} \hat{v}(z, 0) / \partial \varphi^{k-1}, \quad 1 \leq k \leq 2 m, & \\
\text { (3.17) } \sum_{k=1}^{2 m} B_{j 1}^{(1)}\left(0,-z, \frac{\partial}{\partial \varphi}\right) v_{k}(z, 0) \frac{\partial^{k-1} \hat{v}(z, 0)}{\partial \varphi^{k-1}}=0, & 1 \leq j \leq m, \\
\left(3.17^{\prime}\right) \sum_{k=1}^{2 m} B_{j 2}^{(1)}\left(\alpha,-z, \frac{\partial}{\partial \varphi}\right) v_{k}(z, \alpha) \frac{\partial^{k-1} \hat{v}(z, 0)}{\partial \varphi^{k-1}}=0, & 1 \leq j \leq m .
\end{array}
$$

The determinant $\mathscr{D}(z)$ of $(3.17),\left(3.17^{\prime}\right)$ is an entire function of $z$. One can show that condition (2.56) implies that $\mathscr{D}(z)$ is not equal to zero identically. Therefore $\partial^{k-1} \hat{v}(z, 0) / \partial \varphi^{k-1}=0$ almost everywhere, $1 \leq k \leq 2 m$, and so $\hat{v}(z, \varphi)=0$. Therefore we proved the uniqueness of the solution of (3.3), (3.1), (3.2) and consequently that $\operatorname{Im} R_{0}=\operatorname{ker} A_{0} \cap H_{1 / 2, N_{1}}^{(1)}$ for $N_{1} \geq 2 \mathrm{~m}$.

Now we shall show that $\operatorname{Im} R_{0}=\operatorname{ker} A_{0} \cap H_{1 / 2,0}^{(1)}$; that is, when $N_{1}=0$.

Proposition 3.1. Operator $\mathscr{A}$ is bounded from $\operatorname{ker} A_{0} \cap H_{1 / 2, N_{1}}^{(1)}(G)$ to $\mathscr{H}_{0, N_{1}}$ where $N_{1} \geq 0$ is arbitrary:

$$
\left[p_{1}^{+}\left(i \frac{\partial}{\partial x_{1}}-i 0\right)^{s-m_{j 1}-1 / 2} B_{j 1} \Lambda_{-}^{1 / 2-s} l u\right]_{0, N_{1}}^{+} \leq C\left\|\Lambda_{0}^{1 / 2} l u\right\|_{0, N_{1}},
$$




$$
\begin{gathered}
{\left[p_{2}^{-}\left(-i \frac{\partial}{\partial x_{1}} \cos \alpha-i \frac{\partial}{\partial x_{2}} \sin \alpha+i 0\right)^{s-m_{j 2}-1 / 2} B_{j 2} \Lambda_{-}^{1 / 2-s} l u\right]_{0, N_{1}}^{+}} \\
\leq C\left\|\Lambda_{0}^{1 / 2} l u\right\|_{0, N_{1}}
\end{gathered}
$$

for any $u \in \operatorname{ker} A_{0} \cap H_{1 / 2, N_{1}}^{(1)}(G)$.

Proof. Consider the case when $s>m_{j k}+\frac{1}{2}$. There exist "minus"-operators $E_{k}\left(i \partial / \partial x_{1}, i \partial / \partial x_{2}\right), k=1,2$, with the following properties (see [10]):

$$
\xi_{1} E_{1}\left(\xi_{1}, \xi_{2}\right)+\xi_{2} E_{2}\left(\xi_{1}, \xi_{2}\right)=1,
$$

where $E_{k}\left(\xi_{1}, \xi_{2}\right)$ are $C^{\infty}$ (for $|\xi| \neq 0$ ) homogeneous symbols of degree -1 , $\left|E_{k}\right| \leq c /|\xi|, k=1,2$. Let $p$ be an integer, $p \geq\left|\frac{1}{2}-s\right|$. We shall use the identity

$$
1=\left(\xi_{1} E_{1}+\xi_{2} E_{2}\right)^{2 m p}=\sum_{k_{1}+k_{2}=2 m p} c_{k_{1} k_{2}} E_{1}^{k_{1}} E_{2}^{k_{2}} \xi_{1}^{k_{1}} \xi_{2}^{k_{2}}
$$

We have

$$
\begin{aligned}
p_{1}^{+}( & \left.i \frac{\partial}{\partial x_{1}}-i 0\right)^{s-m_{j 1}-1 / 2} B_{j 1} \Lambda_{-}^{1 / 2-s} l u \\
= & \sum_{k_{1}+k_{2}=2 m p} p_{1}^{+}\left(i \frac{\partial}{\partial x_{1}}-i 0\right)^{s-m_{j 1}-1 / 2} \\
& \cdot B_{j 1} c_{k_{1} k_{2}} E_{1}^{k_{1}} E_{2}^{k_{2}} \Lambda_{-}^{-|1 / 2-s|} l\left(\left(i \frac{\partial}{\partial x_{1}}\right)^{k_{1}}\left(i \frac{\partial}{\partial x_{2}}\right)^{k_{2}} u\right),
\end{aligned}
$$

where $l$ means an arbitrary extension. Note that all operators in (3.20) are "minus"-operators. Substitute $i \partial / \partial x_{2}=-\operatorname{cotan} \alpha / 2\left(i \partial / \partial x_{1}\right)+(\csc \alpha / 2) \Lambda_{-}$ in (3.20) and (2.1).

It follows from (2.1) that

$$
\left(i \frac{\partial}{\partial x_{1}}\right)^{2 m j} u=\sum_{k_{1}+k_{2}=2 m j} c_{k_{1} k_{2}}^{(1)}\left(i \frac{\partial}{\partial x_{1}}\right)^{k_{1}} \Lambda_{-}^{k_{2}} u \text { in } G,
$$

where $0 \leq k_{1} \leq(2 m-1) j, 1 \leq j \leq p$, so that $k_{2} \geq j$.

Now substituting (3.21) into (3.20) and fixing an extension in the right-hand side of (3.20) by taking an extension $l u \in H_{1 / 2, N_{1}}^{(1)}\left(\mathbf{R}^{2}\right)$ we cancel all negative powers of $\Lambda_{-}$. From now on the proof of (3.18) will be the same as the usual proof of the inequality

$$
\left[p_{1}^{+} F^{-1}\left|\xi_{1}\right|^{-\varepsilon}|\xi|^{\varepsilon} \tilde{v}(\xi)\right]_{0, N_{1}} \leq C\left\|\Lambda_{0}^{1 / 2} v\right\|_{0, N_{1}}, \quad \varepsilon>0, N_{1} \geq 0 .
$$

When $s \leq m_{j 1}+\frac{1}{2}$ we take $p>\left|s-m_{j 1}-\frac{1}{2}\right|+\left|\frac{1}{2}-s\right|$, use (3.21) and (2.1) to cancel negative powers of $\xi_{1}$ and $\Lambda_{-}$(if any), and then apply (3.22). The estimate $\left(3.18^{\prime}\right)$ is proven analogously. 
Let $u$ be an arbitrary function belonging to $\operatorname{ker} A_{0} \cap H_{1 / 2,0}^{(1)}(G)$. We want to show that there exists $F^{(0)} \in \mathscr{H}_{0,0}$ such that $u=R_{0} F^{(0)}$. Let $l u \in H_{1 / 2,0}^{(1)}\left(\mathbf{R}^{2}\right)$ be an extension of $u$. We have

$$
A_{0}\left(i \frac{\partial}{\partial x_{1}}, i \frac{\partial}{\partial x_{2}}\right) l u=g_{-}
$$

where supp $g_{-} \subset \overline{C G}$ and

$$
\left\|\Lambda_{0}^{-2 m+1 / 2} g_{-}\right\|_{0}^{2}=\int_{\mathbf{R}^{2}}|\xi|^{-4 m+1}\left|\tilde{g}_{-}(\xi)\right|^{2} d \xi<+\infty .
$$

Here $C G$ is the complement of $G$. There exists a sequence $g_{n}^{-} \in \stackrel{\circ}{H}_{M}(C G)$, $\forall M>0$, such that

$$
\left\|\Lambda_{0}^{-2 m+1 / 2}\left(g_{-}-g_{n}^{-}\right)\right\|_{0} \rightarrow 0 \text { as } n \rightarrow \infty
$$

(see, for example, the proof of Theorem 4.1 and Lemma 4.3 in [3]). Let $\tilde{\varphi}_{n}(\xi) \in$ $C_{0}^{\infty}\left(\mathbf{R}^{2}\right)$ be such that

$$
\begin{gathered}
\tilde{\varphi}_{n}(\xi)=0 \quad \text { for }|\xi| \geq 1 / n, \\
\int_{\mathbf{R}^{2}} \tilde{\varphi}_{n}(\xi) d \xi=1, \quad \int_{\mathbf{R}^{2}}\left|\tilde{\varphi}_{n}(\xi)\right| d \xi \leq C, \\
\int_{\mathbf{R}^{2}} \tilde{\varphi}_{n}(\xi) \xi^{k} d \xi=0, \quad 1 \leq|k| \leq 2 m, \\
\int_{\mathbf{R}^{2}}\left(\frac{\partial^{p}}{\partial \xi^{p}} \tilde{\varphi}_{n}(-\xi)\right) \tilde{g}_{n}^{-}(\xi) d \xi=0, \quad 0 \leq|p| \leq 2 m-2 .
\end{gathered}
$$

Then one can show that

$$
u_{n}=A_{0}^{-1}\left(\varphi_{n}(x) g_{n}^{-}(x)\right)
$$

satisfies $p_{G} A_{0} u_{n}=0, u_{n} \in H_{1 / 2, N_{1}}^{(1)}\left(\mathbf{R}^{n}\right)$ for any $N_{1} \geq 0$ and

$$
\left\|\Lambda_{0}^{1 / 2}\left(l u-u_{n}\right)\right\|_{0} \rightarrow 0 \text {. }
$$

Therefore there exists $F_{n}^{(0)} \in \mathscr{H}_{0, N_{1}}, N_{1} \geq 2 m$, such that

$$
p_{G} u_{n}=R_{0} F_{n}^{(0)} \text {. }
$$

Consider a boundary value problem of the form (2.7), (2.8) such that (2.56) and (2.61) are satisfied. Since we proved that $\mathscr{A}$ is bounded from $\operatorname{ker} A_{0} \cap H_{1 / 2,0}^{(1)}(G)$ to $\mathscr{H}_{0,0}$ we have that $\mathscr{A}_{n}=F_{n}$ converges in $\mathscr{H}_{0,0}$ to $\mathscr{A} u=F \in \mathscr{H}_{0,0}, F_{n}=$ $\Phi F_{n}^{(0)}$. Here $\Phi$ is an invertible operator in $\mathscr{H}_{0,0}, \mathscr{A} R_{0}=\Phi$. Therefore $u=$ $R_{0} F^{(0)}$ where $F^{(0)}=\Phi^{-1} \mathscr{A} u=\Phi^{-1} F$ since $\Phi^{-1}$ is bounded in $\mathscr{H}_{0,0}$ and $R_{0}$ is bounded from $\mathscr{K}_{0,0}$ to $\operatorname{ker} A_{0} \cap H_{1 / 2,0}^{(1)}(G)$. We proved that $\operatorname{Im} R_{0}=\operatorname{ker} A_{0} \cap$ $H_{1 / 2,0}^{(1)}(G)$. Let $u \in \operatorname{ker} A_{0} \cap H_{1 / 2, N}^{(1)}(G)$ for some $N>0$. Since $H_{1 / 2, N}^{(1)}(G) \subset$ $H_{1 / 2,0}^{(1)}(G)$ there exists $F^{(0)} \in \mathscr{H}_{0,0}$ such that $u=R_{0} F^{(0)}$. Applying some 
operator $\mathscr{A}$ satisfying (2.56), (2.61) we obtain that $\Phi^{-1} \mathscr{A} u=F^{(0)}$. Since $u \in H_{1 / 2, N}^{(1)}(G)$ then also $F^{(0)} \in \mathscr{H}_{0, N}$. Therefore $\operatorname{Im} R_{0}=\operatorname{ker} A_{0} \cap H_{1 / 2, N}^{(1)}(G)$ for any $N \geq 0$.

Remark 3.1. The proof that $\operatorname{Im} R_{0}=\operatorname{ker} A_{0} \cap H_{1 / 2,0}^{(1)}$ can be used to remove the restriction $N>0$ in Lemma 2.3 in [5]. Therefore Theorems 3.1, 4.1, 5.1 in [5] are valid for $N \geq 0$.

We summarize the main results of $\S 2$ and 3 .

Theorem 3.2. Operator $\mathscr{A}$ is bounded from $\operatorname{ker} A_{0} \cap H_{1 / 2, N}^{(1)}(G)$ to $\mathscr{H}_{0, N}$ for any $N \geq 0$. Assuming that conditions (2.56) and (2.61) are satisfied, we have that $R=R_{0} \Phi^{-1}$ is a bounded operator from $\mathscr{H}_{0, N}$ to $\operatorname{ker} A_{0} \cap H_{1 / 2, N}^{(1)}(G)$ and $R$ is the left and right inverse of $\mathscr{A}$.

Remark 3.2. Using that $R$ is the left and right inverse of $\mathscr{A}$ we can prove the Fredholm property and the regularity results for the operator $\mathscr{A}_{s}$ defined by equations (1.1) and (1.2) by the same method of "freezing" coefficients as in [3, $\S 25]$. We shall not consider this approach in detail in this paper because these results will be proved in the next section in a different way.

Remark 3.3. Using that $R$ is the left inverse of $\mathscr{A}$, using the regularity results, and expanding coefficients of $(1.1),(1.2)$ by the Taylor formula at the vertex $P_{k}$, we can obtain an asymptotic expansion of the solution $u \in H_{s, N_{1}}(\mathscr{D})$ near the vertex $P_{k}$ assuming that the right-hand sides are sufficiently smooth. The proof is very similar to those of [3, §27, pp. 309-315] (see also [4] and [3, §13] for the case of constant coefficients; for another derivation of such asymptotics see [7]). We shall consider the asymptotic expansions and the problems mentioned in Remark 3.2 in detail in another paper.

\section{CONSTRUCTION OF THE RIGHT PARAMETRIX}

4.1. In this section we start the construction of an operator $R_{s}$ bounded from $\mathscr{H}_{s, N_{\mathrm{l}}}$ to $H_{s, N_{\mathrm{l}}}(\mathscr{D})$ such that

$$
\mathscr{A}_{s} R_{s}=I+T \text {, }
$$

where $\mathscr{A}_{s}$ is the operator defined by (1.1) and (1.2), I is the identity operator, and $T$ is a compact operator in $\mathscr{H}_{s, N_{1}}$. It will follow from (4.1) that $\operatorname{Im} \mathscr{A}_{s}$ is closed and coker $\mathscr{A}_{s}$ is finite dimensional. Operator $R_{s}$ is called the right parametrix or the right regularizer of $\mathscr{A}_{s}$.

4.2. Operator $R_{s}^{(1)}$. Let $\psi_{k}\left(x_{1}, x_{2}\right)$ be a $C_{0}^{\infty}$ function in $\mathbf{R}^{2}$ with support in a small neighborhood of $\bar{\Gamma}_{k}$ and such that $\psi_{k}=1$ in a smaller neighborhood of $\Gamma_{k}$. On supp $\psi_{k}$ we introduce local coordinates $\left(y_{1}^{(k)}, y_{2}^{(k)}\right)$ where $\left|y_{2}^{(k)}\right|$ is distance to $l \Gamma_{k}, l \Gamma_{k}$ is a smooth extension of $\bar{\Gamma}_{k}, y_{2}^{(k)}>0$ when $\left(y_{1}^{(k)}, y_{2}^{(k)}\right)$ is inside $\mathscr{D}$, and $\left(y_{1}^{(k)}, 0\right) \in \Gamma_{k}$. Also we shall assume that $y_{1}^{(k)}$ is the arc length of $\Gamma_{k}$ changing from 0 to $a_{k}>0$ when the point on $\Gamma_{k}$ changes from $P_{k-1}$ to $P_{k}, 1 \leq k \leq N, P_{0}=P_{N}$. 
Denote by $R_{s}^{(1)}$ the operator

$$
R_{s}^{(1)}(g, c)=p_{\mathscr{D}} A_{0}^{(-1)}\left(x, i \frac{\partial}{\partial x}\right) l_{0} g+p_{\mathscr{D}} \sum_{k=1}^{N} \sum_{j=1}^{m} \psi_{k} R_{k j} c_{k j},
$$

where $p_{\mathscr{D}}$ is the restriction operator to $\mathscr{D}, l_{0} g$ is some fixed extension operator from $\mathscr{D}$ to $\mathbf{R}^{2}$ such that $l_{0} g=0$ for large $|x|, g \in H_{s-2 m, N_{1}}(\mathscr{D})$ and

$$
\left\|l_{0} g\right\|_{s-2 m, N_{1}} \leq 2\|g\|_{s-2 m, N_{1}}^{+},
$$

$A_{0}^{(-1)}$ is a pseudodifferential operator in $\mathbf{R}^{2}$ with symbol $A_{0}^{-1}(x, \xi)$ for $|\xi|>$ $1, c_{k j} \in H_{0, N_{1}}\left(\Gamma_{k}\right)$, and operators $R_{k j}$ will be defined later.

Denote by $\Lambda_{-}^{1 / 2-s}(x, \xi)$ the following symbol:

$$
\Lambda_{-}^{1 / 2-s(x)}(x, \xi)=\left(a_{1}(x) \xi_{1}+a_{2}(x) \xi_{2}-i 0\right)^{-s(x)+1 / 2}
$$

for $x$ belonging to a small neighborhood $\mathscr{D}_{\varepsilon}$ of $\partial \mathscr{D}$ in $\mathbf{R}^{2}$, where $\left(a_{1}(x)\right.$, $\left.a_{2}(x)\right)$ is a $C^{\infty}$ vector having an acute angle with the interior normal to $\mathscr{D}$ at any point of $\bar{\Gamma}_{k}, k=1, \ldots, N, s(x)=s$ in $\mathscr{D}_{\varepsilon}, s(x)=\frac{1}{2}$, and $\Lambda_{-}^{1 / 2-s}(x, \xi)=$ 1 outside $\mathscr{D}_{2 \varepsilon}, s(x) \in C^{\infty}\left(\mathbf{R}^{2}\right)$. form:

Operator $R_{k j}$ expressed in $y^{(k)}=\left(y_{1}^{(k)}, y_{2}^{(k)}\right)$ coordinates has the following

$$
\begin{aligned}
& R_{k j} c_{k j}=\frac{1}{2 \pi} \int_{-\infty}^{\infty} e^{-i y_{2}^{(k)} \lambda_{k j}\left(y^{(k)}, \eta_{1}^{(k)}\right)} \Lambda_{-, k}^{1 / 2-s}\left(y^{(k)},\right. \eta_{1}^{(k)}, \lambda_{k j}\left(y^{(k)}, \eta_{1}^{(k)}\right) \\
& \cdot \widetilde{c_{k j}^{+}}\left(\eta_{1}^{(k)}\right) e^{-i y_{1}^{(k)} \eta_{1}^{(k)}} d \eta_{1}^{(k)},
\end{aligned}
$$

where $\Lambda_{-, k}^{1 / 2-s}\left(y^{(k)}, \eta_{1}^{(k)}, \eta_{2}^{(k)}\right)$ is the symbol $\Lambda_{-}^{1 / 2-s(x)}$ written in $y^{(k)}$ coordinates, $\lambda_{k j}\left(y^{(k)}, \eta_{1}^{(k)}\right)$ is the same as in $\left(1.6^{\prime}\right), c_{k j}^{+}\left(y_{1}^{(k)}\right)=c_{k j}\left(y_{1}^{(k)}\right)$ for $0 \leq$ $y_{1}^{(k)} \leq a_{k}, c_{k j}^{+}\left(y_{1}^{(k)}\right)=0$ for $y_{1}^{(k)} \notin\left[0, a_{k}\right], \eta^{(k)}=\left(\eta_{1}^{(k)}, \eta_{2}^{(k)}\right)$ are the dual coordinates to $y^{(k)}$, and $\widetilde{c_{k j}^{+}}\left(\eta_{1}^{(k)}\right)$ is the Fourier transform of $c_{k j}^{+}\left(y_{1}^{(k)}\right)$.

Remark 4.1. (4.5) defines $R_{k j} c_{k j}$ for $y_{2}^{(k)} \geq 0$. Denote by $G_{k j}\left(y^{(k)}, x_{2}, x_{1}-t\right)$ the kernel of $R_{k j}$ for $s=\frac{1}{2}$ :

$$
G_{k j} c_{k j}=\int_{0}^{a_{k}} G_{k j}\left(y^{(k)}, y_{2}^{(k)}, y_{1}^{(k)}-t\right) c_{k j}(t) d t .
$$

It follows from (2.23) that $G_{k j}\left(y^{(k)}, x_{2}, x_{1}-t\right)$ is real analytic for $\left(x_{1}, x_{2}\right) \in$ supp $\psi_{k} \backslash \bar{\Gamma}_{k}$. Note that (2.77) gives an expression for the Fourier transform of $G_{k j}\left(y^{(k)}, x_{2}, x_{1}\right)$ in $\left(x_{1}, x_{2}\right)$. In the case when condition

$$
\left(\operatorname{supp} \psi_{k} \cap \overline{\mathscr{D}}\right) \subset\left\{y^{(k)}, y_{2}^{(k)} \geq 0\right\}
$$


is not satisfied we shall use formulas of the form (2.23), (2.77). One should also modify (4.5) to

$$
R_{k j} c_{k j}=\Lambda_{-, k}^{1 / 2-s}\left(y^{(k)}, i \partial / \partial y^{(k)}\right) G_{k j} c_{k j} .
$$

Note that the principal parts of (4.5) and (4.8) coincide on supp $\psi_{k}$. Analogously to Remark 2.1 , one can show that all results are exactly the same whether (4.7) holds or not. Therefore we shall consider for simplicity only the case when (4.7) is satisfied.

Note that $\Lambda_{-}^{1 / 2-s(x)}(i \partial / \partial x)$ is a "minus"-operator with respect to domain $\mathscr{D}$; that is, $p_{\mathscr{D}} \Lambda_{-}^{1 / 2-s(x)} l g$ does not depend on the extension of $g$ from $\mathscr{D}$ to $\mathbf{R}^{2}$. Also, near $\Gamma_{k}$ we have

$$
\Lambda_{-, k}^{1 / 2-s}\left(y^{(k)}, \eta^{(k)}\right)=\left(a_{1 k}\left(y^{(k)}\right) \eta_{1}^{(k)}+a_{2 k}\left(y^{(k)}\right) \eta_{2}^{(k)}-i 0\right)^{1 / 2-s},
$$

where $a_{2 k}\left(y^{(k)}\right)>0$ and so that $\Lambda_{-, k}^{1 / 2-s}$ is analytic in $\eta_{2}^{(k)}$ for $\operatorname{Im} \eta_{2}^{(k)}<0$. Since $\operatorname{Im} \lambda_{k j} \neq 0$ for $\eta_{1}^{(k)} \neq 0, \Lambda_{-}^{1 / 2-s}\left(y^{(k)}, \eta_{1}^{(k)}, \lambda_{k j}\left(y^{(k)}, \eta_{1}^{(k)}\right)\right)$ is an elliptic symbol in $\eta_{1}^{(k)}$.

Therefore estimates analogous to those in $[3, \S 24]$ show that $R_{k j}$ is a bounded operator from $H_{0, N_{1}}\left(\Gamma_{k}\right)$ to $H_{s, N_{1}}(\mathscr{D})$. Also, it follows from [3, §24] that $p_{\mathscr{D}} A_{0}^{(-1)} l_{0} f$ is bounded from $H_{s-2 m, N_{1}}(\mathscr{D})$ to $H_{s, N_{1}}(\mathscr{D})$. Therefore $R_{s}^{(1)}$ is a bounded operator from $\mathscr{H}_{s, N_{1}}^{(1)}=H_{s-2 m, N_{1}}(\mathscr{D}) \times \prod_{k, j} H_{0, N_{1}}\left(\Gamma_{k}\right)$ to $H_{s, N_{1}}(\mathscr{D})$.

4.3. Operator $\mathscr{A}_{s}^{(1)}$. Let $\varphi_{k 1}\left(y_{1}^{(k)}\right)$ and $\varphi_{k 2}\left(y_{1}^{(k)}\right)$ be $C^{\infty}$ functions such that $\varphi_{k 1}\left(y_{1}^{(k)}\right)=0$ for $y_{1}^{(k)}>\frac{2}{3} a_{k}, \varphi_{k 2}\left(y_{1}^{(k)}\right)=0$ for $y_{1}^{(k)}<\frac{1}{3} a_{k}$, and $\varphi_{k 1}+\varphi_{k 2}=1$.

Denote by $\Lambda_{1, k}^{\alpha}$ an elliptic operator in $\mathbf{R}^{1}$ with the symbol

$$
\Lambda_{1, k}^{\alpha}\left(y_{1}^{(k)}, \eta_{1}^{(k)}\right)=\left(\eta_{1}^{(k)}-i 0\right)^{\alpha \varphi_{k 1}\left(y_{1}^{(k)}\right)}\left(\eta_{1}^{(k)}+i 0\right)^{\alpha \varphi_{k 2}\left(y_{1}^{(k)}\right)} \text {. }
$$

Note that $\Lambda_{1, k}^{\alpha}$ is a "minus"-operator with respect to $\Gamma_{k}$ in the sense that $v_{-}=0$ for $y_{1}^{(k)} \notin\left[0, a_{k}\right]$ implies $\Lambda_{1, k}^{\alpha} v_{-}=0$ for $y_{1}^{(k)} \notin\left[0, a_{k}\right]$. Therefore $p_{\Gamma_{k}} \Lambda_{1, k}^{\alpha} l g$ is independent of an extension of $g$ from $\Gamma_{k}$ to $\mathbf{R}^{1}$ where $p_{\Gamma_{k}}$ is the restriction operator to $\Gamma_{k}$. Applying $\Lambda_{1, k}^{s-m_{k j}-1 / 2}$ to the boundary conditions (1.2) we obtain

$$
p_{\Gamma_{k}} \Lambda_{1, k}^{s-m_{k j}-1 / 2} B_{k j} l u=p_{\Gamma_{k}} \Lambda_{1, k}^{s-m_{k j}-1 / 2} l_{0} h_{k j}, \quad 1 \leq k \leq N, 1 \leq j \leq m
$$

$l_{0} h_{k j}$ is a fixed extension operator from $\Gamma_{k}$ to $\mathbf{R}^{1}$ such that $l_{0} h_{k j}$ has a compact support in $\mathbf{R}^{1}$ and

$$
\left[l_{0} h_{k j}\right]_{s-m_{k j}-1 / 2, N_{1}} \leq 2\left[h_{k j}\right]_{s-m_{k j}-1 / 2, N_{1}}^{+} \text {. }
$$

In (4.11) one should first take the restriction of $B_{k j} l u$ to $l \Gamma_{k}=\mathbf{R}^{1}$ and then apply $p_{\Gamma_{k}} \Lambda_{1, k}^{s-m_{k j}-1 / 2}$. Note that

$$
h_{k j}^{(1)}=p_{\Gamma_{k}} \Lambda_{1, k}^{s-m_{k j}-1 / 2} l_{0} h_{k j} \in H_{0, N_{1}}\left(\Gamma_{k}\right) .
$$


The operator $\Lambda_{1, k}^{s-m_{k j}-1 / 2}$ is a Fredholm operator from $H_{s-m_{k j}-1 / 2, N_{1}}\left(\Gamma_{k}\right)$ to $H_{0, N_{1}}\left(\Gamma_{k}\right)$. Indeed, we have

$$
\begin{aligned}
& \Lambda_{1, k}^{s-m_{k j}-1 / 2} \Lambda_{1, k}^{-\left(s-m_{k j}-1 / 2\right)}=I+T_{1}, \\
& \Lambda_{1, k}^{-\left(s-m_{k j}-1 / 2\right)} \Lambda_{1, k}^{s-m_{k j}-1 / 2}=I+T_{2},
\end{aligned}
$$

where $I$ is the identity operator and $T_{1}, T_{2}$ are compact operators. Moreover, the index of $\Lambda_{1, k}^{s-m_{k j}-1 / 2}$ is zero. This follows, for example, from the fact that if we introduce $i \tau, \tau>0$, instead of $i 0$ in (4.10), then for large $\tau$ we obtain that $\Lambda_{1 k \tau}^{s-m_{k j}-1 / 2}$ is an invertible operator since $T_{1}, T_{2}$ will have a small norm for $\tau>0$ large. Since the norm of operator $\Lambda_{1 k \tau}^{s-m_{k j}-1 / 2}$ depends continuously on $\tau$, we obtain that the index of $\Lambda_{1, k}^{s-m_{k j}-1 / 2}$ is zero. Therefore the problem of the Fredholm property and of the index for the operator $\mathscr{A}_{s}$ defined by (1.1) and (1.2) is the same as for the operator defined by (1.1) and (4.11). We shall denote the operator defined by (1.1), (4.11) by $\mathscr{A}_{s}^{(1)}$.

4.4. Composition $\mathscr{A}_{s}^{(1)} R_{s}^{(1)}$. Now apply $\mathscr{A}_{s}^{(1)}$ to $R_{s}^{(1)}$. We obtain

$$
p_{\mathscr{D}} A\left(x, i \frac{\partial}{\partial x}\right) p_{\mathscr{D}} A_{0}^{(-1)}\left(x, i \frac{\partial}{\partial x}\right) l_{0} g=g+T_{0} l_{0} g,
$$

where $T_{0}$ is a bounded operator from $H_{s-2 m, N_{1}}\left(\mathbf{R}^{2}\right)$ to $H_{s-2 m+1, N_{1}}(\mathscr{D})$. Also, we have in coordinates $y^{(k)}$ :

$$
\begin{aligned}
& p_{\mathscr{D}} A_{k}\left(y^{(k)}, i \frac{\partial}{\partial y^{(k)}}\right) \psi_{k} R_{k j} c_{k j} \\
& =p_{\mathscr{D}} \psi_{k} \frac{1}{2 \pi} \int_{-\infty}^{\infty} \frac{A_{k 0}\left(y^{(k)}, \eta_{1}^{(k)}, \lambda_{k j}\left(y^{(k)}, \eta_{1}^{(k)}\right)\right)}{\Lambda_{-, k}^{s-1 / 2}\left(y^{(k)}, \eta_{1}^{(k)}, \lambda_{k j}\left(y^{(k)}, \eta_{1}^{(k)}\right)\right)} \\
& \cdot e^{-i y_{1}^{(k)} \eta_{1}^{(k)}} \widetilde{c_{k j}^{+}}\left(\eta_{1}^{(k)}\right) d \eta_{1}^{(k)}+T_{k j} c_{k j},
\end{aligned}
$$

where $T_{k j}$ are bounded operators from $H_{0, N_{1}}\left(\Gamma_{k}\right)$ to $H_{s-2 m+1, N_{1}}(\mathscr{D})$.

The principal term in the right-hand side of (4.16) is equal to zero since

$$
A_{k 0}\left(y^{(k)}, \eta_{1}^{(k)}, \lambda_{k j}\left(y^{(k)}, \eta_{1}^{(k)}\right)\right)=0 \text {. }
$$

We shall call operators analogous to $T_{k j}, T_{0}$ the smoothing operators. Therefore we have

$$
p_{\mathscr{D}} A(x, D) R_{s}^{(1)}(g, c)=g+T_{0} l_{0} g+\sum_{k, j} T_{k j} c_{k j} .
$$

Now substitute $R_{s}^{(1)}$ into the boundary conditions (4.11). Note that

$$
p_{\Gamma_{k}} \Lambda_{1, k}^{s-m_{k j}-1 / 2} B_{k j} A_{0}^{(-1)} l_{0} g \in H_{0, N_{1}}\left(\Gamma_{k}\right) \text {. }
$$

We have

$$
p_{\Gamma_{k}} \Lambda_{1, k}^{s-m_{k j}-1 / 2} B_{k j} \psi_{k} R_{k r} c_{k r}=p_{\Gamma_{k}} b_{k j r} c_{k r}^{+}+T_{k j k r} c_{k r}
$$


where $T_{k j k r}$ are bounded from $H_{0, N_{\mathrm{l}}}\left(\Gamma_{k}\right)$ to $H_{1, N_{\mathrm{l}}}\left(\Gamma_{k}\right) ; b_{k j r}$ are pseudodifferential operators in $\mathbf{R}^{1}$ :

$$
b_{k j r} c_{k r}^{+}=\frac{1}{2 \pi} \int_{-\infty}^{\infty} b_{k j r}\left(y_{1}^{(k)}, \eta_{1}^{(k)}\right) e^{-i y_{1}^{(k)} \eta_{1}^{(k)}} \widetilde{c_{k r}^{+}}\left(\eta_{1}^{(k)}\right) d \eta_{1}^{(k)},
$$

where

$$
\left.b_{k j r}\left(y_{1}^{(k)}, \eta_{1}^{(k)}\right)=\frac{B_{k j 0}\left(y_{1}^{(k)}, 0, \eta_{1}^{(k)}, \lambda_{k j}\left(y_{1}^{(k)}, 0, \eta_{1}^{(k)}\right)\right)}{\Lambda_{-, k}^{s-1 / 2}\left(y_{1}^{(k)}, 0, \eta_{1}^{(k)}, \lambda_{k j}\left(y_{1}^{(k)}, 0, \eta_{1}^{(k)}\right)\right)} \Lambda_{1, k}^{s-m_{k j}-1 / 2}\left(y_{1}^{(k)}, \eta_{1}^{(k)}\right)\right) \text {. }
$$

$B_{k j 0}$ is the same as in (1.7). Note that $\operatorname{deg}_{\eta_{1}^{(k)}} b_{k j r}=0$. Therefore $b_{k j r}$ can be represented in the form

$$
b_{k j r}\left(y_{1}^{(k)}, \eta_{1}^{(k)}\right)=b_{k j r}^{+}\left(y_{1}^{(k)}\right) \theta\left(\eta_{1}^{(k)}\right)+b_{k j r}^{-}\left(y_{1}^{(k)}\right) \theta\left(-\eta_{1}^{(k)}\right),
$$

where

$$
\begin{gathered}
b_{k j r}^{ \pm}\left(y_{1}^{(k)}\right)=b_{k j r}\left(y_{1}^{(k)}, \pm 1\right), \\
\theta(t)=1 \quad \text { for } t>0, \quad \theta(t)=0 \text { for } t<0 .
\end{gathered}
$$

Therefore (4.20) can be rewritten in the form

$$
b_{k j r} c_{k r}^{+}=b_{k j r}^{+}\left(y_{1}^{(k)}\right) \Pi^{-} c_{k r}^{+}+b_{k j r}^{-}\left(y_{1}^{(k)}\right) \Pi^{+} c_{k 2}^{+},
$$

where $\Pi^{\mp} c_{k r}^{+}=F^{-1}\left(\theta\left( \pm \eta_{1}^{(k)}\right) \widetilde{c_{k r}^{+}}\left(\eta_{1}\right)\right)$ are the Cauchy-type integrals (see [3, §5 and 2]),

$$
\Pi^{ \pm} c_{k r}^{+}= \pm \frac{i}{2 \pi} \int_{-\infty}^{\infty} \frac{c_{k r}^{+}(t) d t}{y_{1}^{(k)} \pm i 0-t} .
$$

Note that the Shapiro-Lopatinsky condition (1.7) is equivalent to the condition

$$
\operatorname{det}\left\|b_{k j r}^{ \pm}\left(y_{1}^{(k)}\right)\right\|_{j, r=1}^{m} \neq 0, \quad 0 \leq y_{1}^{(k)} \leq a_{k}, k=1, \ldots, N .
$$

Now we shall find $p_{\Gamma_{k}} \Lambda_{1, k}^{s-m_{k j}-1 / 2} B_{k j} \psi_{n} R_{n r} c_{n r}$ for $n \neq k$. The kernel $R_{n r}\left(y^{(n)}, y_{2}^{(n)}, y_{1}^{(n)}-t\right)$ of the operator $R_{n r}$ is $C^{\infty}$ for $\left|y_{2}^{(n)}\right|+\left|y_{1}^{(n)}-t\right|>0$, that is, when $\left(y_{1}^{(n)}, y_{2}^{(n)}\right) \notin \Gamma_{n}$. Therefore we can get nonsmoothing operators only when $k-n= \pm 1$. Moreover, since the kernel of $R_{n r}$ is $O\left(1 /\left(\left|y_{2}^{(n)}\right|+\left|y_{1}^{(n)}-t\right|\right)\right)$, the only contribution to the nonsmoothing operator comes from the vertices $P_{k-1}$ and $P_{k}, 1 \leq k \leq N, P_{0}=P_{N}$. Indeed we obtain this nonsmoothing operator if we freeze coefficients of $A, B_{k-1, j}, B_{k j}$ at $P_{k-1}$ and consider a constant coefficient problem with homogeneous principal terms in a corner formed by the tangent lines to $\Gamma_{k-1}$ and $\Gamma_{k}$ at the vertex $P_{k-1}$. Then we obtain as in $\S 2$ a nonsmoothing operator similar to the operator in (2.36) acting on $c_{2}=\left(c_{12}, \ldots, c_{m 2}\right)$. We shall call such operators the Mellin operators. 
We have

$$
\begin{aligned}
p_{\Gamma_{k}} \Lambda_{1, k}^{s-m_{k j}-1 / 2} B_{k j} & \left(p_{\mathscr{D}} \sum_{n=1}^{N} \sum_{r=1}^{m} \psi_{n} R_{n r} c_{n r}\right) \\
= & p_{\Gamma_{k}}\left(\sum_{r=1}^{m} b_{k j r} c_{k r}^{+}+\sum_{r=1}^{m} \varphi_{k 1} M_{k j, k-1, r} c_{k-1, r}\right. \\
& \left.+\sum_{r=1}^{m} \varphi_{k 2} M_{k j, k+1, r} c_{k+1, r}+\sum_{n=1}^{N} \sum_{r=1}^{m} T_{k j n r} c_{n r}\right) \\
= & h_{k j}^{(1)}-p_{\Gamma_{k}} \Lambda_{1, k}^{s-m_{k j}-1 / 2} B_{k j} A_{0}^{(-1)} l_{0} g, \quad 1 \leq k \leq N, 1 \leq j \leq m,
\end{aligned}
$$

where $T_{k j n r}$ are smoothing operators, i.e., operators bounded from $H_{0, N_{1}}\left(\Gamma_{n}\right)$ to $H_{1, N_{1}}\left(\Gamma_{k}\right), b_{k j r}$ are pseudodifferential operators (see (4.20) and (4.24)), and $M_{k j, k \pm 1, r}$ are the Mellin operators that we shall describe now in detail. Denote by $\alpha_{k-1}$ the angle between the tangent lines to $\Gamma_{k}$ and $\Gamma_{k-1}$ at the vertex $P_{k-1}, 1 \leq k \leq N$. Note that the Jacobi matrix $\mathscr{D} y^{(k-1)} / \mathscr{D} y^{(k)}$ at $P_{k-1}$ is equal to $U_{\pi-\alpha_{k-1}}(\operatorname{see}(2.5),(2.9))$. Also, the coordinates of $P_{k-1}$ are $(0,0)$ in $y^{(k)}=\left(y_{1}^{(k)}, y_{2}^{(k)}\right)$ coordinates and $\left(0, a_{k-1}\right)$ in $y^{(k-1)}$ coordinates.

Denote by $B_{k j, k \pm 1}\left(y^{(k \pm 1)}, \eta^{(k \pm 1)}\right)$ the principal symbol of $B_{k j}$ in $y^{(k \pm 1)}$ coordinates. We have

$$
M_{k j, k-1, r} c_{k-1, r}=\int_{0}^{a_{k-1}} M_{k j, k-1, r}\left(y_{1}^{(k)}, t_{k-1}-a_{k-1}\right) c_{k-1, r}\left(t_{k-1}\right) d t_{k-1},
$$

$$
M_{k j, k+1, r} c_{k+1, r}=\int_{0}^{a_{k+1}} M_{k j, k+1, r}\left(y_{1}^{(k)}-a_{k}, t_{k+1}\right) c_{k+1, r}\left(t_{k+1}\right) d t_{k+1},
$$

where

$$
\begin{aligned}
M_{k j, k-1, r}( & \left.y_{1}^{(k)}, t_{k-1}-a_{k-1}\right) \\
=\frac{1}{2 \pi} & \int_{-\infty}^{\infty} \frac{B_{k j, k-1}\left(a_{k-1}, 0, \eta_{1}^{(k-1)}, \lambda_{k-1, r}\left(\eta_{1}^{(k-1)}\right)\right)}{\Lambda_{-, k-1}^{s-1 / 2}\left(a_{k-1}, 0, \eta_{1}^{(k-1)}, \lambda_{k-1, r}\left(\eta_{1}^{(k-1)}\right)\right)} \\
& \cdot\left(-\eta_{1}^{(k-1)} \cos \alpha_{k-1}+\lambda_{k-1, r}\left(\eta_{1}^{(k-1)}\right) \sin \alpha_{k-1}-i 0\right)^{s-m_{k j}-1 / 2} \\
& \cdot e^{-i y_{1}^{(k)} \sin \alpha_{k-1} \lambda_{k-1, r}\left(\eta_{1}^{(k)}\right)} e^{-i \eta_{1}^{(k-1)}\left(-y_{1}^{(k)} \cos \alpha_{k-1}+a_{k-1}-t_{k-1}\right)} d \eta_{1}^{(k-1)}
\end{aligned}
$$

and analogously

$$
\begin{aligned}
M_{k j, k+1, r}( & \left.y_{1}^{(k)}-a_{k}, t_{k+1}\right) \\
=\frac{1}{2 \pi} & \int_{-\infty}^{\infty} \frac{B_{k j, k+1}\left(0,0, \eta_{1}^{(k+1)}, \lambda_{k+1, r}\left(\eta_{1}^{(k+1)}\right)\right)}{\Lambda_{-, k+1}^{s-1 / 2}\left(0,0, \eta_{1}^{(k+1)}, \lambda_{k+1, r}\left(\eta_{1}^{(k+1)}\right)\right)} \\
& \cdot\left(-\eta_{1}^{(k+1)} \cos \alpha_{k}-\lambda_{k+1, r}\left(\eta_{1}^{(k+1)}\right) \sin \alpha_{k}+i 0\right)^{s-m_{k}-1 / 2} \\
& \cdot e^{i\left(y_{1}^{(k)}-a_{k}\right) \sin \alpha_{k} \lambda_{k+1, r}\left(\eta_{1}^{(k+1)}\right)} e^{-i \eta_{1}^{(k+1)}\left(-\left(y_{1}^{(k)}-a_{k}\right) \cos \alpha_{k}-t_{k+1}\right)} d \eta_{1}^{(k+1)},
\end{aligned}
$$


where

$$
\begin{array}{cc}
A_{k-1,0}\left(a_{k-1}, 0,1, \lambda_{k-1, r}\right)=0, & 1 \leq r \leq 2 m, \\
A_{k+1,0}\left(0,0,1, \lambda_{k+1, r}\right)=0, & 1 \leq r \leq 2 m, \\
\lambda_{k \pm 1, r}\left(\eta_{1}^{(k \pm 1)}\right)=\lambda_{k \pm 1, r} \eta_{1}^{(k \pm 1)} \quad \text { for } \eta_{1}^{(k \pm 1)}>0, \\
\lambda_{k \pm 1, r}\left(\eta_{1}^{(k+1)}\right)=\lambda_{k \pm 1, r+m} \eta_{1}^{(k \pm 1)} \quad \text { for } \eta_{1}^{(k \pm 1)}<0 .
\end{array}
$$

Computing integrals in $\eta_{1}^{(k \pm 1)}$ in (4.30) and (4.31) we obtain (4.34)

$$
\begin{aligned}
M_{k j, k-1, r}\left(y_{1}^{(k)}, t_{k-1}-a_{k-1}\right) \\
=\frac{1}{2 \pi i} \sum_{p, r=1}^{m} b_{k j p 1}^{+} \frac{1}{y_{1}^{(k)}\left(\lambda_{k-1, p} \sin \alpha_{k-1}-\cos \alpha_{k-1}\right)+a_{k-1}-t_{k-1}} \\
\quad-\frac{1}{2 \pi i} \sum_{p, r=1}^{m} b_{k j p 1}^{-} \frac{1}{y_{1}^{(k)}\left(\lambda_{k-1, p+m} \sin \alpha_{k-1}-\cos \alpha_{k-1}\right)+a_{k-1}-t_{k-1}},
\end{aligned}
$$

where

$$
\begin{aligned}
b_{k j p 1}^{ \pm}= & B_{k j 0}\left(0,0, \pm 1, \lambda_{k, p}( \pm 1)\right) \\
& \cdot\left( \pm a_{1 k}(0,0) \pm a_{2 k}(0,0) \lambda_{k, p}( \pm 1)-i 0\right)^{1 / 2-s}(-1 \pm i 0)^{s-m_{k j}-1 / 2},
\end{aligned}
$$

$$
A_{k 0}\left(0,0,1, \lambda_{k, r}\right)=0, \quad 1 \leq r \leq 2 m, \lambda_{k, r}(+1)=\lambda_{k r}, \lambda_{k r}(-1)=-\lambda_{k, r+m} .
$$

Note that $b_{k j p 1}^{ \pm}$are the same as in $(2.31)$ if we choose $\left(a_{1 k}(0,0), a_{2 k}(0,0)\right)=$ $\left(\cos \left(\alpha_{k-1} / 2\right), \sin \left(\alpha_{k-1} / 2\right)\right)$ and we used formulas of the form (2.34) and (2.35) relating expressions in $y^{(k)}$ and $y^{(k-1)}$ coordinates. Analogously

$$
\begin{aligned}
M_{k j, k+1, r}\left(y_{1}^{(k)}-a_{k}, t_{k+1}\right) \\
=\frac{1}{2 \pi i} \sum_{p, r=1}^{m} b_{k j p 2}^{+} \frac{1}{-\left(y_{1}^{(k)}-a_{k}\right) \lambda_{k+1, p} \sin \alpha_{k}-\left(y_{1}^{(k)}-a_{k}\right) \cos \alpha_{k}-t_{k+1}} \\
\quad-\frac{1}{2 \pi i} \sum_{p, r=1}^{m} b_{k j p 2}^{-} \frac{1}{-\left(y_{1}^{(k)}-a_{k}\right) \lambda_{k+1, p+m} \sin \alpha_{k}-\left(y_{1}^{(k)}-a_{k}\right) \cos \alpha_{k}-t_{k+1}},
\end{aligned}
$$

where $b_{k j p 2}^{ \pm}$are the same as $b_{k j p 1}^{ \pm}$but evaluated at the point $\left(0, a_{k}\right)$ instead of $(0,0)$. (Note that $(0,0)$ are coordinates of the vertex $P_{k-1}$ in $y^{(k)}$ coordinates and $\left(0, a_{k}\right)$ are coordinates of $P_{k}$ in the same system of coordinates.) To construct a right regularizer for $\mathscr{A}_{s}^{(1)}$ it is enough to prove that (4.27) defines a Fredholm operator in $\prod_{k=1}^{N} \prod_{j=1}^{m} H_{0, N_{1}}\left(\Gamma_{k}\right)$ and to find a right regularizer for this operator. This will be done in the next section. 


\section{Algebra OF OPERATORS CONTAINING} SINGULAR INTEGRAL OPERATORS ON AN INTERVAL

The system (4.27) can be rewritten as a particular case of the following system of equations:

$$
b_{+}(t) \Pi_{0}^{-} u+b_{-}(t) \Pi_{0}^{+} u+\varphi_{0}(t) M_{0} u+\varphi_{1}(t) M_{1} u+T u=h(t), \underset{t \in[0,1],}{ }
$$

where $b_{ \pm}(t)$ are $L \times L C^{\infty}$ matrices on $[0,1], \varphi_{1}(t) \in C^{\infty}\left(\mathbf{R}^{1}\right), \varphi_{1}=0$ for $t<\frac{1}{3}, \varphi_{1}=1$ for $t>\frac{2}{3}, \varphi_{0}=1-\varphi_{1}, T$ is a compact operator in $H_{0, N_{1}}(0,1)$,

$$
\begin{gathered}
\Pi_{0}^{ \pm} u= \pm \frac{i}{2 \pi} \int_{0}^{1} \frac{u(\tau)}{t \pm i 0-\tau} d \tau, \quad 0<t<1, \\
M_{0} u=\int_{0}^{1} m_{0}\left(\frac{t}{\tau}\right) \frac{u(\tau)}{\tau} d \tau \\
M_{1} u=\int_{0}^{1} m_{1}\left(\frac{t-1}{\tau-1}\right) \frac{u(\tau)}{\tau-1} d \tau
\end{gathered}
$$

We assume that $m_{k}(t), k=0,1$, are matrices having the following properties (see $[3, \S \S 2$ and 15]):

$$
\begin{aligned}
& \left|m_{k}(t)\right| \leq \frac{c}{t^{\delta}(1+t)^{1-2 \delta}}, \quad 0<\delta<\frac{1}{2}, 0<t<+\infty, \\
& \left|t^{j} \frac{d^{j}}{d t^{j}} m_{k}(t)\right| \leq \frac{c_{j}}{t^{\delta}(1+t)^{1-2 \delta}}, \quad \forall j \geq 0,0<t<+\infty .
\end{aligned}
$$

We shall call operators (5.3), (5.4) Mellin operators. Note that this class of kernels $m_{k}(t)$ is much larger than we need. It would be enough to consider a smaller class analogous to the class considered in [4]. Note that close results were also obtained in [1].

In this section $H_{0, N_{1}}(0,1)$ means the Sobolev space with norm

$$
[u]_{0, N_{1}}^{2}=\sum_{k=0}^{N_{1}}\left[t^{k}(1-t)^{k} \frac{d^{k}}{d t^{k}} u(t)\right]_{0}^{+},
$$

where $[v]_{0}^{+}$is the norm in $L_{2}(0,1)$. Note that Mellin operators $M_{0}, M_{1}$ are bounded from $H_{0,0}(0,1)=L_{2}(0,1)$ to $H_{0, N_{1}}(0,1)$ for any $N_{1} \geq 0$. We shall find conditions when the operator $\Phi$ defined by (5.1) is Fredholm in $H_{0, N_{1}}(0,1)$. In order to rewrite (4.27) in the form (5.1) we shall assume without loss of generality that $N$ is even. Otherwise one should add artificially to $\Gamma_{N}$ an additional vertex dividing $\Gamma_{N}$ into two parts. Then we shall make the change of variables

(5.6) $y_{1}^{(k)}=a_{k} t, \quad t_{1}^{(k)}=a_{k} \tau, \quad 0 \leq t \leq 1,0 \leq \tau \leq 1$, when $k$ is odd

and

$\left(5.6^{\prime}\right) \quad y_{1}^{(k)}=a_{k}(1-t), \quad t_{1}^{(k)}=a_{k}(1-\tau)$,

$$
0 \leq t \leq 1,0 \leq \tau \leq 1 \text {, when } k \text { is even }
$$


Note that the reverse of the direction change $\Pi_{0}^{+}$to $\Pi_{0}^{-}$and $\Pi_{0}^{-}$to $\Pi_{0}^{+}$:

$$
\begin{aligned}
\Pi_{0}^{+} u & =\frac{i}{2 \pi} \int_{0}^{1} \frac{u(\tau)}{t-\tau+i 0} d \tau=\frac{i}{2 \pi} \int_{0}^{1} \frac{u\left(1-\tau^{\prime}\right) d \tau^{\prime}}{\left(1-t^{\prime}\right)-\left(1-\tau^{\prime}\right)+i 0} \\
& =-\frac{i}{2 \pi} \int_{0}^{1} \frac{u\left(1-\tau^{\prime}\right) d \tau^{\prime}}{t^{\prime}-\tau^{\prime}-i 0}=\Pi_{0}^{-} u_{1},
\end{aligned}
$$

where $t^{\prime}=1-t, \tau^{\prime}=1-\tau, u_{1}(t)=u(1-t)$. Analogously

$$
\Pi_{0}^{-} u=\Pi_{0}^{+} u_{1}, \quad u_{1}(t)=u(1-t) .
$$

We have

$$
\Pi_{0}^{+}\left(\Pi_{0}^{+} u\right)=\Pi_{0}^{+} u+\frac{1}{4 \pi} \int_{0}^{1}\left(\ln \frac{1-t}{1-\tau}-\ln \frac{t}{\tau}\right) \frac{u(\tau)}{t-\tau} d \tau
$$

Indeed

$$
\Pi_{0}^{+}\left(\Pi_{0}^{+} u\right)=\lim _{\varepsilon_{1} \rightarrow 0, \varepsilon_{2} \rightarrow 0}\left(\frac{i}{2 \pi}\right)^{2} \int_{0}^{1} \frac{1}{t+i \varepsilon_{1}-y}\left(\int_{0}^{1} \frac{1}{y+i \varepsilon_{2}-\tau} u(\tau) d \tau\right) d y
$$

Integrating in $y$ we obtain

$$
\begin{aligned}
\Pi_{0}^{+}\left(\Pi_{0}^{+} u\right)= & \lim _{\varepsilon_{1} \rightarrow 0, \varepsilon_{2} \rightarrow 0}\left(\frac{i}{2 \pi}\right)^{2} \int_{0}^{1} \frac{1}{t+i\left(\varepsilon_{1}+\varepsilon_{2}\right)-\tau} \\
& \cdot\left(\left.\ln \left(y+i \varepsilon_{2}-\tau\right)\right|_{0} ^{1}-\left.\ln \left(y-t-i \varepsilon_{1}\right)\right|_{0} ^{1}\right) u(\tau) d \tau,
\end{aligned}
$$

where $\ln z$ is the branch of logarithm that is real for positive $z$. Therefore

$$
\left.\ln \left(y+i \varepsilon_{2}-\tau\right)\right|_{0} ^{1} \rightarrow \ln (1-\tau)-i \pi-\ln \tau \quad \text { as } \varepsilon_{2} \rightarrow 0
$$

and

$$
\left.\ln \left(y-t-i \varepsilon_{1}\right)\right|_{0} ^{1} \rightarrow \ln (1-t)-\ln t+i \pi \quad \text { as } \varepsilon_{1} \rightarrow 0 .
$$

Therefore we obtain

$$
\Pi_{0}^{+}\left(\Pi_{0}^{+} u\right)=\Pi_{0}^{+} u-K u
$$

where

$$
K u=\left(\frac{i}{2 \pi}\right)^{2} \int_{0}^{1}\left(\ln \frac{1-t}{1-\tau}-\ln \frac{t}{\tau}\right) \frac{u(\tau)}{t-\tau} d \tau
$$

Note that $K$ is a Mellin operator of the form (5.3), (5.4) with a kernel satisfying (5.5), $\left(5.5^{\prime}\right)$. Analogously one can check that

$$
\begin{aligned}
& \Pi_{0}^{-}\left(\Pi_{0}^{-} u\right)=\Pi_{0}^{-} u-K u, \\
& \Pi_{0}^{+}\left(\Pi_{0}^{-} u\right)=\Pi_{0}^{-}\left(\Pi_{0}^{+} u\right)=K u .
\end{aligned}
$$

Consider a Mellin operator on the half-line $t>0$ :

$$
M u=\int_{0}^{\infty} m\left(\frac{t}{\tau}\right) \frac{u(\tau)}{\tau} d \tau
$$


Applying the Mellin transform, one obtains (see, for example, [3, §15])

$$
\widehat{M u}(z)=\hat{m}(z) \hat{u}(z),
$$

where $\hat{u}(z)$ is the Mellin transform of $u(\tau)$ and

$$
\hat{m}(z)=\int_{0}^{\infty} m(t) t^{z-1} d t .
$$

Note that $(5.5),\left(5.5^{\prime}\right)$ imply that $\widehat{M}(z)$ is an analytic function in the strip $\delta<\operatorname{Re} z<1-\delta$ rapidly decreasing as $|z| \rightarrow \infty$ for $\delta+\varepsilon \leq \operatorname{Re} z \leq 1-\delta+\varepsilon$, $\forall \varepsilon>0$. We shall call $\hat{m}(z)$ the symbol of operator (5.14). Also, we shall call $\hat{m}_{0}(z)$ the symbol of operator (5.3) where $\hat{m}_{0}(z)=\int_{0}^{\infty} m_{0}(t) t^{z-1} d t$. Note that $\left(1-\varphi_{0}(t / \varepsilon)\right) M_{0} u$ and $M_{0}\left(1-\varphi_{0}(\tau / \varepsilon)\right) u(\tau)$ have a $C^{\infty}$ kernel for any $\varepsilon>0$ so that the main contribution to the Mellin operator $M_{0}$ comes from the small neighborhood $0<t<\varepsilon, 0<\tau<\varepsilon$. Analogously we shall call $-\hat{m}_{1}(z)$ where

$$
\hat{m}_{1}(z)=\int_{0}^{\infty} m_{1}(t) t^{z-1} d t
$$

the symbol of Mellin operator (5.4). For example, the symbol of

$$
K_{0} u=\left(\frac{i}{2 \pi}\right)^{2} \int_{0}^{1}\left(\ln \frac{t}{\tau}\right) \frac{u(\tau)}{t-\tau} d \tau
$$

is equal to

$$
\widehat{K_{0}}(z)=\frac{1}{\left(1-e^{2 \pi i z}\right)^{2}}-\frac{1}{1-e^{2 \pi i z}}=\frac{e^{2 \pi i z}}{\left(1-e^{2 \pi i z}\right)^{2}} .
$$

To compute (5.18) we used formulas of the form (5.13), (2.52). Analogously the symbol of

is equal to

$$
K_{1} u=\left(\frac{i}{2 \pi}\right)^{2} \int_{0}^{1} \ln \frac{1-t}{1-\tau} \frac{u(\tau)}{t-\tau} d \tau
$$

$$
\widehat{K_{1}}(z)=-e^{2 \pi i z} /\left(1-e^{2 \pi i z}\right)^{2} .
$$

The minus sign arises because when we convert the Mellin operator of form (5.4) to the operator of form (5.3) by changing $t$ to $1-t^{\prime}$ and $\tau$ to $1-\tau^{\prime}$ we get a minus sign because of $\tau-1=-\tau^{\prime}$. We have (cf. $[3, \S 15]$ and [4]) that the composition of two Mellin operators of the same form (5.3) or (5.4) is again a Mellin operator of the same form modulo $T$, where here and below $T$ means a bounded operator from $H_{0, N_{1}}(0,1)$ to $H_{1, N_{1}}(0,1)$. Also, the symbol of the composition will be the product of symbols and (see [3, §15] and [4]) the compositions $\Pi_{0}^{+} M_{0}, \Pi_{0}^{-} M_{0}, M_{0} \Pi_{0}^{+}, M_{0} \Pi_{0}^{-}$are Mellin operators of the form (5.3) modulo $T$ with symbols

$$
\begin{array}{ll}
-\frac{e^{2 \pi i z}}{1-e^{2 \pi i z}} \hat{m}_{0}(z), & \frac{1}{1-e^{2 \pi i z}} \hat{m}_{0}(z), \\
-\frac{e^{2 \pi i z}}{1-e^{2 \pi i z}} \hat{m}_{0}(z), & \frac{1}{1-e^{2 \pi i z}} \hat{m}_{0}(z),
\end{array}
$$


respectively. If $M_{1}$ has form (5.4) then $\Pi_{0}^{+} M_{1}, \Pi_{0}^{-} M_{1}$ are also operators of form (5.4) modulo $T$ with symbols

$$
-\frac{e^{2 \pi i z}}{1-e^{2 \pi i z}} \hat{m}_{1}(z), \quad \frac{1}{1-e^{2 \pi i z}} \hat{m}_{1}(z) .
$$

Therefore operators (5.1) form an algebra. To any operator of form (5.1) we shall assign a symbol in the following way: Consider an infinite "rectangle" with vertices (clockwise) at $(0,-\infty),(0,+\infty),(1,+\infty),(1,-\infty)$. Denote by $\gamma$ the boundary of this "rectangle." On the top horizontal side we assign $b_{+}(t)$, $0 \leq t \leq 1$, on the bottom horizontal side we assign $b_{-}(t), 0 \leq t \leq 1$, and on the left vertical side we assign

$$
M_{0}(z)=-\frac{e^{2 \pi i z}}{1-e^{2 \pi i z}} b_{-}(0)+\frac{1}{1-e^{2 \pi i z}} b_{+}(0)+\hat{m}_{0}(z),
$$

where $z=\frac{1}{2}+i \tau,-\infty \leq \tau \leq+\infty ; \tau=-\infty$ corresponds to the vertex $(0,-\infty)$ and $\tau=+\infty$ corresponds to the vertex $(0,+\infty)$. Here $\hat{m}_{0}(z)$ is the symbol of $M_{0}$ and (5.19) is the contribution of the left endpoint of $[0,1]$ to the symbol. Finally, on the right vertical line we assign

$$
M_{1}(z)=\frac{1}{1-e^{2 \pi i z}} b_{-}(1)-\frac{e^{2 \pi i z}}{1-e^{2 \pi i z}} b_{+}(1)-\hat{m}_{1}(z),
$$

$z=\frac{1}{2}+i \tau$, where $\tau=-\infty$ corresponds to the vertex $(1,+\infty)$ of the "rectangle" and $\tau=+\infty$ corresponds to the vertex $(1,-\infty)$. Therefore $(5.20)$ is the contribution of the right endpoint of $[0,1]$ to the symbol. Note the difference between (5.19) and (5.20). The explanation of this difference is that the model problem for the right endpoint is the equation of the form

$$
\begin{aligned}
b_{+}(1) \frac{i}{2 \pi} \int_{-\infty}^{1} & \frac{u(\tau)}{t-\tau+i 0} d \tau+b_{-}(1)\left(-\frac{i}{2 \pi}\right) \int_{-\infty}^{1} \frac{u(\tau)}{t-\tau-i 0} d \tau \\
& \quad+\int_{-\infty}^{1} m_{1}\left(\frac{t-1}{\tau-1}\right) \frac{u(\tau)}{\tau-1} d \tau=h(t), \quad-\infty<t<1 .
\end{aligned}
$$

When one transforms this problem to the positive axis changing $t$ to $t^{\prime}=1-t$, then $\Pi^{+}, \Pi^{-}, m_{1}(t)$ change to $\Pi^{-}, \Pi^{+},-m_{1}(t)$, respectively. Therefore we constructed a continuous matrix $\sigma(B)$ on $\gamma$ and we shall call this matrix the symbol of operator $B$ defined by the left-hand side of $(5.1)$. As in $[3, \S 15]$ we have the following theorem:

Theorem 5.1. The symbol of the composition of two operators $B_{1}$ and $B_{2}$ is equal to the product of symbols $\sigma\left(B_{1}\right) \sigma\left(B_{2}\right)$, and the necessary and sufficient condition for the operator $B$ to be a Fredholm operator in $H_{0, N_{1}}(0,1)$ is that $\sigma(B)$ is a nonsingular matrix for any point of $\gamma$; that is, $B$ is Fredholm if

$$
\operatorname{det} b_{+}(t) \neq 0, \quad \operatorname{det} b_{-}(t) \neq 0, \quad 0 \leq t \leq 1,
$$


(5.23)

$$
\begin{aligned}
& \operatorname{det}\left(-\frac{e^{2 \pi i z}}{1-e^{2 \pi i z}} b_{-}(0)+\frac{1}{1-e^{2 \pi i z}} b_{+}(0)+\hat{m}_{0}(z)\right) \neq 0, \\
& z=\frac{1}{2}+i \tau,-\infty \leq \tau \leq+\infty,
\end{aligned}
$$

$$
\begin{aligned}
\operatorname{det}\left(\frac{1}{1-e^{2 \pi i z}} b_{-}(1)-\frac{e^{2 \pi i z}}{1-e^{2 \pi i z}} b_{+}(1)-\hat{m}_{1}(z)\right) & \neq 0, \\
& z=\frac{1}{2}+i \tau,-\infty \leq \tau \leq+\infty .
\end{aligned}
$$

Moreover the index of $B$ is the winding number of $\operatorname{det} \sigma(B)$ counting clockwise:

$$
\text { ind } \begin{aligned}
\sigma(B)= & \left.\frac{1}{2 \pi} \Delta \arg \operatorname{det} \sigma(B)\right|_{\gamma} \\
= & \left.\frac{1}{2 \pi} \Delta \arg \operatorname{det} b_{-}(t)\right|_{t=1} ^{0}+\left.\frac{1}{2 \pi} \Delta \arg \operatorname{det} M_{0}(z)\right|_{\tau=-\infty} ^{\infty} \\
& +\left.\frac{1}{2 \pi} \Delta \arg \operatorname{det} b_{+}(t)\right|_{t=0} ^{1}+\left.\frac{1}{2 \pi} \Delta \arg \operatorname{det} M_{1}(z)\right|_{\tau=-\infty} ^{\infty} .
\end{aligned}
$$

The proof of Theorem 5.1 is the same as in $[3, \S 15]$. Note that we always can apply $b_{+}^{-1}(t)$ to $(5.1)$, just reducing the operator $B$ to the case when $b_{+}(t)=I$ and $b_{-}(t)$ is replaced by $b_{+}^{-1}(t) b_{-}(t)$. Then the symbol of $B$ will be defined on an infinite "triangle" when the top side of the "rectangle" shrinks to a point. In this case the formula for the index has the form

$$
\text { ind } \begin{aligned}
B= & \left.\frac{1}{2 \pi} \Delta \arg \operatorname{det} b_{+}^{-1}(t) b_{-}(t)\right|_{1} ^{0} \\
& +\left.\frac{1}{2 \pi} \Delta \operatorname{argdet} b_{+}^{-1}(0) M_{0}(z)\right|_{\tau=-\infty} ^{\infty} \\
& +\left.\frac{1}{2 \pi} \Delta \arg \operatorname{deg} b_{+}^{-1}(1) M_{1}(z)\right|_{\tau=-\infty} ^{\infty} .
\end{aligned}
$$

We shall apply conditions (5.22), (5.23), (5.24) and formula (5.25) or (5.26) to the system (4.27). Condition (5.22) has the form

$$
\operatorname{det}\left\|b_{k j r}^{ \pm}\left(y_{1}^{(k)}\right)\right\|_{j, r=1}^{m} \neq 0, \quad 0 \leq y_{1}^{(k)} \leq a_{k}, k=1, \ldots, N,
$$

where $b_{k j r}^{ \pm}\left(y_{1}^{(k)}\right)$ are the same as in (4.21), (4.23). Indeed $b_{ \pm}(t)$ are $L \times L$ matrices consisting of $N m \times m$ blocks $\left\|b_{k j r}^{ \pm}\right\|_{j, r=1}^{m}$ along the main diagonal, $L=m N$. Now consider conditions (5.23), (5.24) in the case of system (4.27). Again each of $m N \times m N$ matrices $M_{0}(z)$ and $M_{1}(z)$ consists of $N / 2$ blocks of size $2 m \times 2 m$ along the main diagonal. Each of these $2 m \times 2 m$ matrices $M^{(k-1)}(z)$ corresponds to the contribution of the vertex $P_{k-1}, k=1, \ldots, N$, $P_{0}=P_{N}$, and conditions (5.23), (5.24) are equivalent to the conditions

$\left(5.27^{\prime}\right) \quad \operatorname{det} M^{(k-1)}(z) \neq 0, \quad z=\frac{1}{2}+i \tau,-\infty \leq \tau \leq+\infty, k=1, \ldots, N$. 
We shall write an explicit expression for $M^{(k-1)}(z), 1 \leq k \leq N$. The situation here is the same as in $\S 2$.

Denote

$$
b_{k}^{ \pm}=\left\|b_{k j p 1}^{ \pm}\right\|_{j, p=1}^{m},
$$

where $b_{k j p 1}^{ \pm}$are the same as in (4.35). Analogously

$$
b_{k-1}^{ \pm}=\left\|b_{k-1, j p 1}^{ \pm}\right\|_{j, p=1}^{m},
$$

where $b_{k-1, j p 1}^{ \pm}$are given by $(4.35)$ with $k$ replaced by $k-1,(0,0)$ replaced by $\left(a_{k-1}, 0\right)$, and $( \pm 1-i 0)^{s-m_{k j}-1 / 2}$ replaced by $( \pm 1+i 0)^{s-m_{k-1, j}-1 / 2}$ (see (2.68)). Denote

$$
\Delta_{k 1}(z)=\left\|e^{i \beta_{k p} z} \delta_{j p}\right\|_{j, p=1}^{m}, \quad \Delta_{k 2}(z)=\left\|e^{i \beta_{k, p+m}} \delta_{j p}\right\|_{j, p=1}^{m},
$$

where

$$
\begin{gathered}
i \beta_{k, p}=\ln \left(\cos \alpha_{k-1}+\lambda_{k, p} \sin \alpha_{k-1}\right), \quad 1 \leq k \leq N, 1 \leq p \leq 2 m, P_{0}=P_{N}, \\
0<\arg \left(\cos \alpha_{k-1}+\lambda_{k p} \sin \alpha_{k-1}\right)<2 \pi .
\end{gathered}
$$

Then it follows from (4.34), (4.37), (2.57) that

$$
\begin{aligned}
& M^{(k-1)}(z)=\left(1-e^{2 \pi i z}\right)^{-1} \\
& \cdot\left\|\begin{array}{cc}
\left(I-b_{k} e^{2 \pi i z}\right) & -\left(\Delta_{k 1}(z)-b_{k} \Delta_{k 2}(z)\right)\left(\frac{a_{k-1}}{a_{k}}\right)^{z} \\
e^{2 \pi i z}\left(\Delta_{k 1}^{-1}(z)-b_{k-1} \Delta_{k 2}^{-1}(z)\right)\left(\frac{a_{k}}{a_{k-1}}\right)^{z} & -e^{2 \pi i z} I-b_{k-1}
\end{array}\right\|,
\end{aligned}
$$

where

$$
b_{k}=\left(b_{k}^{+}\right)^{-1} b_{k}^{-}, \quad b_{k-1}=\left(b_{k-1}^{+}\right)^{-1} b_{k-1}^{-} .
$$

Note that factors $\left(a_{k} / a_{k-1}\right)^{z}$ and $\left(a_{k-1} / a_{k}\right)^{z}$ appear because of the change of variables (5.6), $\left(5.6^{\prime}\right)$. As in (2.79) we have

$$
\operatorname{det} M^{(k-1)}(z)=\operatorname{det}\left(-b_{k-1}+e^{2 \pi i z} \Delta_{k 1}^{-1}(z) b_{k} \Delta_{k 2}(z)\right) \operatorname{det} \Delta_{k 3}(z),
$$

where

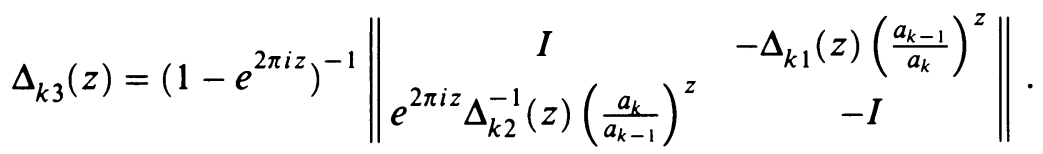

Without loss of generality we shall assume that

$$
\operatorname{det} \Delta_{k 3}(z)=\left(1-e^{2 \pi i z}\right)^{-1} \prod_{j=1}^{m}\left(-1+e^{i\left(2 \pi-\beta_{k, j+m}+\beta_{k j}\right) z}\right) \neq 0 .
$$

Otherwise we should change the operatcr (4.5) (see Remark 2.2). Note that (5.36) is always satisfied when $A_{0}\left(x, \xi_{1}, \xi_{2}\right)$ has real coefficients. Then we have (see $(2.82)$ )

$$
2 \pi-\beta_{k, j+m}+\beta_{k j}=2 \operatorname{Re} \beta_{k j}, \quad 0<\operatorname{Re} \beta_{k j}<2 \pi .
$$


Therefore condition $\left(5.27^{\prime}\right)$ is reduced to the following condition:

(5.38) $\operatorname{det}\left(-b_{k-1}+e^{2 \pi i z} \Delta_{k 1}^{-1}(z) b_{k} \Delta_{k 2}(z)\right) \neq 0$,

$$
z=\frac{1}{2}+i \tau,-\infty<\tau<+\infty, 1 \leq k \leq N .
$$

Analogously to (2.73) we shall simplify condition (5.38) to make the dependence of $s$ more explicit.

Denote

$$
\begin{gathered}
b_{k 0}^{ \pm}=\left\|B_{k j 0}\left(0,0, \pm 1, \lambda_{k p}( \pm 1)\right)\right\|_{j, p=1}^{m}, \\
a_{k}^{ \pm}=\left\|\left( \pm a_{1 k}(0,0) \pm a_{2 k}(0,0) \lambda_{k p}( \pm 1)-i 0\right)^{1 / 2-s} \delta_{j p}\right\|_{j, p=1}^{m} .
\end{gathered}
$$

Analogously

$$
b_{k-1,0}^{ \pm}=\left\|B_{k-1, j 0}\left(0, a_{k-1}, \pm 1, \lambda_{k-1, p}( \pm 1)\right)\right\|_{j, p=1}^{m},
$$

$$
a_{k-1}^{ \pm}=\left\|\left( \pm a_{1, k-1}\left(0, a_{k-1}\right) \pm a_{2, k-1}\left(0, a_{k-1}\right) \lambda_{k-1, p}( \pm 1)-i 0\right)^{1 / 2-s} \delta_{j p}\right\|_{j, p=1}^{m} .
$$

Since

$$
a_{1, k-1} \eta_{1}^{(k-1)}+a_{2, k-1} \eta_{2}^{(k-1)}=a_{1, k} \eta_{1}^{(k)}+a_{2, k} \eta_{2}^{(k)}, \eta^{(k-1)}=U_{\pi-\alpha_{k-1}} \eta^{(k)}
$$

and

$$
\lambda_{k-1, p}=\frac{\sin \alpha_{k-1}-\lambda_{k, p} \cos \alpha_{k-1}}{-\cos \alpha_{k-1}-\lambda_{k, p} \sin \alpha_{k-1}}
$$

(see (2.20)), we obtain

$$
\begin{aligned}
a_{1, k-1} & +a_{2, k-1} \lambda_{k-1, p}=-a_{1 k} \cos \alpha_{k-1}-a_{2 k} \sin \alpha_{k-1} \\
& +\frac{\sin \alpha_{k-1}-\lambda_{k, p} \cos \alpha_{k-1}}{-\cos \alpha_{k-1}-\lambda_{k, p} \sin \alpha_{k-1}}\left(a_{1 k} \sin \alpha_{k-1}-a_{2 k} \cos \alpha_{k-1}\right) \\
= & \left(a_{1 k}+a_{2 k} \lambda_{k, p}\right)\left(-\cos \alpha_{k-1}-\lambda_{k p} \sin \alpha_{k-1}\right)^{-1} .
\end{aligned}
$$

Therefore

$$
\begin{aligned}
\left( \pm a_{1, k-1} \pm a_{2, k-1} \lambda_{k-1, p}( \pm 1)-i 0\right)^{1 / 2-s} & \\
= & \left( \pm a_{1 k} \pm a_{2 k} \lambda_{k p}( \pm 1)-i 0\right)^{1 / 2-s} e^{i\left(\pi-\beta_{k, p}^{ \pm}\right)(1 / 2-s)}
\end{aligned}
$$

since

and

$$
-\pi<\arg \left( \pm a_{1, k-1} \pm a_{2, k-1} \lambda_{k-1, p}( \pm 1)\right)<0
$$

$$
0<\arg e^{i \beta_{k, p}}=\arg \left(\cos \alpha_{k-1}+\lambda_{k, p} \sin \alpha_{k-1}\right)<2 \pi .
$$

Here we denote $\beta_{k, p}^{+}=\beta_{k p}, \beta_{k p}^{-}=\beta_{k, p+m}$. We have

$$
\begin{gathered}
b_{k}^{+}=b_{k 0}^{+} a_{k}^{+}, \quad b_{k}^{-}=\left\|e^{-i \pi\left(s-m_{k j}-1 / 2\right)} \delta_{j p}\right\| b_{k 0}^{-} a_{k}^{-}, \\
b_{k-1}^{+}=b_{k 0}^{+} a_{k}^{+} e^{i \pi(1 / 2-s)} \Delta_{k 1}^{-1}\left(\frac{1}{2}-s\right), \\
b_{k-1}^{-}=\left\|e^{i \pi\left(s-m_{k-1, j}-1 / 2\right)} \delta_{j p}\right\| b_{k-1,0}^{-} a_{k}^{-} e^{i \pi(1 / 2-s)} \Delta_{k 2}^{-1}\left(\frac{1}{2}-s\right) .
\end{gathered}
$$


Substituting (5.43), (5.44), (5.45) into (5.38) and multiplying from the left by $\operatorname{det} a_{k}^{+}$and from the right by $\operatorname{det}\left(a_{k}^{-}\right)^{-1}$, we obtain that $(5.38)$ is equivalent to the condition

$$
\begin{aligned}
\operatorname{det}\left(-\Delta_{k 1}\right. & \left(\frac{1}{2}-s\right)\left(b_{k-1,0}^{+}\right)^{-1}\left\|e^{-i \pi m_{k-1, j}} \delta_{j p}\right\| b_{k-1,0}^{-} \Delta_{k 2}^{-1}\left(\frac{1}{2}-s\right) e^{i \pi(s-1 / 2)} \\
& \left.+e^{2 \pi i z} \Delta_{k 1}^{-1}(z)\left(b_{k 0}^{+}\right)^{-1}\left\|e^{-i \pi m_{k, j}} \delta_{j p}\right\| b_{k 0}^{-} \Delta_{k 2}(z) e^{-i \pi(s-1 / 2)}\right) \neq 0 .
\end{aligned}
$$

Dividing by $\operatorname{det} \Delta_{k 1}\left(\frac{1}{2}-s\right) e^{i \pi(s-1 / 2)}$ and $\operatorname{det} \Delta_{k 2}^{-1}\left(\frac{1}{2}-s\right)$, we finally obtain the following condition:

$$
\operatorname{det} M_{k-1,0}\left(z-s+\frac{1}{2}\right) \neq 0, \quad z=\frac{1}{2}+\tau,-\infty<\tau<+\infty,
$$

where

$$
\begin{aligned}
& M_{k-1,0}\left(z-s+\frac{1}{2}\right) \\
& =\left(-\left(b_{k-1,0}^{+}\right)^{-1}\left\|e^{-i \pi m_{k-1, j}} \delta_{j p}\right\| b_{k-1,0}^{-}\right. \\
& \quad+e^{2 \pi i(z-s+1 / 2)} \Delta_{k 1}^{-1}\left(z-s+\frac{1}{2}\right)\left(b_{k 0}^{+}\right)^{-1} \\
& \left.\quad \cdot\left\|e^{-i \pi m_{k j}} \delta_{j p}\right\| b_{k 0}^{-} \Delta_{k 2}\left(z-s+\frac{1}{2}\right)\right), \quad 1 \leq k \leq N .
\end{aligned}
$$

Denote by $\Sigma_{k-1}, 1 \leq k \leq N$, the set of zeros of $\operatorname{det} M_{k-1,0}(z)$ (see (5.48)). Then $s \in \mathbf{R}$ satisfies (5.47) iff

$$
1-s \notin \operatorname{Re} \Sigma_{k-1} \text {. }
$$

We shall summarize the main results of this section:

Theorem 5.2. Operator $\Phi_{s}$ defined by the left-hand sides of (4.17), (4.27) is Fredholm in $\mathscr{H}_{0, N_{1}}$ iff (5.27) and (5.49) are satisfied for all $k=1, \ldots, N$. The formula for the index has the form

$$
\text { ind } \begin{aligned}
\Phi_{s}= & \left.\sum_{k=1}^{N} \frac{1}{2 \pi} \Delta \arg \operatorname{det}\left(b_{k}^{+}\left(y_{1}^{(k)}\right)\right)^{-1} b_{k}^{-}\left(y_{1}^{(k)}\right)\right|_{y_{1}^{(k)}=a_{k}} ^{0} \\
& +\left.\sum_{k=1}^{N} \frac{1}{2 \pi} \Delta \arg \operatorname{det} M^{(k-1)}(z)\right|_{1 / 2-i \infty} ^{1 / 2+i \infty},
\end{aligned}
$$

where

$$
b_{k}^{ \pm}\left(y_{1}^{(k)}\right)=\left\|b_{k j p}^{ \pm}\left(y_{1}^{(k)}\right)\right\|_{j, p=1}^{m}
$$

are the same as in (4.21), (4.23) and $M^{(k-1)}(z)$ are the same as in (5.23). Note that the direction in (5.50) is clockwise with respect to $\mathscr{D}$.

Remark 5.1. In Theorem 5.2 we assumed that condition (5.36) is satisfied for $1 \leq k \leq N$. Otherwise one should change operators (4.5) (see Remark 2.2). Then operator $\Phi_{s}$ will change and (5.27), (5.49) will be the necessary and sufficient conditions for the Fredholmity of this operator. Note that the final result concerning the Fredholmity of $\mathscr{A}_{s}$ will need only the condition (1.7) that 
is equivalent to (5.27) and the condition (5.49). Also note that if one needs to add an additional vertex to make $N$ even then the condition $\left(5.27^{\prime}\right)$ for such a vertex is always satisfied and also the contribution of this vertex to the index is equal to zero.

Example 5.1. Consider the case when $\alpha_{k}=\pi, k=1, \ldots, N$, that is, the case of mixed elliptic boundary value problems in a smooth domain (see $[3, \S 24]$ ). Then we have

$$
\begin{gathered}
\beta_{k p}=\frac{1}{i} \ln \left(\cos \alpha_{k-1}+\lambda_{k, p} \sin \alpha_{k-1}\right)=\pi, \quad 1 \leq p \leq 2 m, \\
\Delta_{k 1}(z)=\Delta_{k 2}(z)=e^{i \pi z} I,
\end{gathered}
$$

where $I$ is the identity matrix. Also (see (5.36))

$$
\operatorname{det} \Delta_{k 3}(z)=-\left(-1+e^{2 \pi i z}\right)^{m-1}
$$

and (see (5.34))

$$
\begin{aligned}
-\left(1-e^{2 \pi i z}\right)^{-1}\left(-b_{k-1}+e^{2 \pi i z} b_{k}\right) & =\left(\frac{1}{1-e^{2 \pi i z}} b_{k-1}-\frac{e^{2 \pi i z}}{1-e^{2 \pi i z}} b_{k}\right) \\
& =\left(\frac{1}{1+e^{-2 \pi \tau}} b_{k-1}+\frac{e^{-2 \pi \tau}}{1+e^{-2 \pi \tau}} b_{k}\right),
\end{aligned}
$$

where

$$
b_{k-1}=\left(b_{k-1}^{+}\left(a_{k-1}\right)\right)^{-1} b_{k-1}^{-}\left(a_{k-1}\right), b_{k}=\left(b_{k}^{+}(0)\right)^{-1} b_{k}^{-}(0) \text {, }
$$

$b_{p}^{ \pm}\left(y_{1}^{(p)}\right)$ are the same as in (5.50), $p=k-1, k$. Note that when $\tau$ changes from $-\infty$ to $+\infty$ then $\left(1-e^{2 \pi i z}\right)^{-1}\left(b_{k-1}-e^{2 \pi i z} b_{k}\right)$ describes a line segment in the space of matrices connecting $b_{k-1}$ and $b_{k}$. Note that this line segment does not intersect 0 because of $(5.38)$.

Consider a discontinuous matrix function on $\partial \mathscr{D}$ equal to

$$
\left(b_{k}^{+}\left(y_{1}^{(k)}\right)\right)^{-1} b_{k}^{-}\left(y_{1}^{(k)}\right) \text { on } \Gamma_{k}, k=1, \ldots, N .
$$

Connecting $b_{k-1}$ with $b_{k}$ by a line segment, we obtain a continuous curve. Then (5.50) implies that ind $\Phi_{s}$ is equal to the winding number of the determinant of this closed curve counted clockwise. We shall prove in $\S 7$ that ind $\Phi_{s}$ is equal to the index of operator $\mathscr{A}_{s}$ defined by the boundary value problem (1.1), (1.2). In the next section we shall find another expression for ind $\Phi_{s}$ that makes the dependence on $s$ more explicit.

Note that there is an easier approach to the computation of the index of mixed boundary value problems based on the study of the Riemann-Hilbert 
problem with discontinuous coefficients (see $[3, \S 14$ and (15.47)], where a similar problem was treated).

\section{CoMputation OF THE INDEX}

In this section we shall find simpler formulas for the index of operator $\Phi_{s}$. It will be proved in $\S 7$ that operator $R_{s}^{(1)}$ (see (4.2)) is always Fredholm and has zero index. Therefore ind $\Phi_{s}=$ ind $\mathscr{A}_{s}$.

We have (see (4.21), (4.9), (4.10)) that

$$
\begin{aligned}
\operatorname{det}\left\|b_{k j p}^{+}\left(y_{1}^{(k)}\right)\right\|= & \operatorname{det} \| B_{k j 0}\left(y_{1}^{(k)}, 0,1, \lambda_{k p}\left(y_{1}^{(k)}, 0\right) \|\right. \\
& \cdot \prod_{p=1}^{m}\left(a_{k}\left(y_{1}^{(k)}, 0\right)+a_{2 k}\left(y_{1}^{(k)}, 0\right) \lambda_{k p}\left(y_{1}^{(k)}, 0\right)-i 0\right)^{1 / 2-s},
\end{aligned}
$$

$$
\begin{aligned}
\operatorname{det}\left\|b_{k j p}^{-}\left(y_{1}^{(k)}\right)\right\|= & \operatorname{det} \| B_{k j 0}\left(y_{1}^{(k)}, 0,-1,-\lambda_{k, p+m}\left(y_{1}^{(k)}, 0\right) \|\right. \\
& \cdot \prod_{p=1}^{m}\left(-a_{1 k}\left(y_{1}^{(k)}, 0\right)-a_{2 k}\left(y_{1}^{(k)}, 0\right) \lambda_{k, p+m}\left(y_{1}^{(k)}, 0\right)-i 0\right)^{1 / 2-s} \\
& \cdot \prod_{j=1}^{m} e^{-i \pi\left(s-m_{k j}-1 / 2\right) \varphi_{k 1}\left(y_{1}^{(k)}\right)+i \pi\left(s-m_{k j}-1 / 2\right) \varphi_{k 2}\left(y_{1}^{(k)}\right)} .
\end{aligned}
$$

It is clear that

$$
\begin{gathered}
\left.\frac{1}{2 \pi} \Delta \arg \prod_{j=1}^{m} e^{-i \pi\left(s-m_{k j}-1 / 2\right) \varphi_{k 1}\left(y_{1}^{(k)}\right)+i \pi\left(s-m_{k j}-1 / 2\right) \varphi_{k 2}\left(y_{1}^{(k)}\right)}\right|_{y_{1}^{(k)}=a_{k}} ^{0} \\
=-\sum_{j=1}^{m}\left(s-m_{k j}-1 / 2\right) .
\end{gathered}
$$

Note that $a_{1 k}\left(y_{1}^{(k)}, 0\right)+a_{2 k}\left(y_{1}^{(k)}, 0\right) \lambda_{k, p}\left(y_{1}^{(k)}, 0\right)$ belongs to the lower complex half-plane for all $y_{1}^{(k)}, 0 \leq y_{1}^{(k)} \leq a_{k}$, since $\operatorname{Im} a_{2 k}\left(y_{1}^{(k)}, 0\right) \lambda_{k, p}\left(y_{1}^{(k)}, 0\right)<0$. Therefore the increment of the argument of

$$
\left(a_{1 k}\left(y_{1}^{(k)}, 0\right)+a_{2 k}\left(y_{1}^{(k)}, 0\right) \lambda_{k p}\left(y_{1}^{(k)}, 0\right)-i 0\right)^{1 / 2-s} \text { on } \Gamma_{k}
$$

is equal to the difference of the arguments at the endpoints $P_{k-1}$ and $P_{k}$. Note that in $y^{(k)}$ system of coordinates the coordinates of $P_{k-1}$ are $(0,0)$ and the coordinates of $P_{k}$ are $\left(a_{k}, 0\right)$. So

$$
\begin{aligned}
\frac{1}{2 \pi} \Delta & \left.\arg \left(a_{1 k}\left(y_{1}^{(k)}, 0\right)+a_{2 k}\left(y_{1}^{(k)}, 0\right) \lambda_{k p}\left(y_{1}^{(k)}, 0\right)-i 0\right)^{1 / 2-s}\right|_{y_{1}^{(k)}=a_{k}} ^{0} \\
& =\frac{1}{2 \pi}\left(\varphi_{k p}^{+}\left(P_{k-1}\right)-\varphi_{k p}^{+}\left(P_{k}\right)\right)(1 / 2-s),
\end{aligned}
$$


where $\varphi_{k p}^{+}\left(P_{i}\right)$ is the argument of

$$
\begin{gathered}
a_{1 k}\left(y_{1}^{(k)}, 0\right)+a_{2 k}\left(y_{1}^{(k)}, 0\right) \lambda_{k p}\left(y_{1}^{(k)}, 0\right) \quad \text { at } P_{i}, \\
-\pi<\varphi_{k p}^{+}\left(P_{i}\right)<0, \quad i=k-1, k .
\end{gathered}
$$

Analogously

$$
\begin{aligned}
\frac{1}{2 \pi} \Delta & \left.\arg \left(-a_{1 k}\left(y_{1}^{(k)}, 0\right)-a_{2 k}\left(y_{1}^{(k)}, 0\right) \lambda_{k, p+m}\left(y_{1}^{(k)}, 0\right)-i 0\right)^{1 / 2-s}\right|_{y_{1}^{(k)}=a_{k}} ^{0} \\
& =\frac{1}{2 \pi}\left(\varphi_{k p}^{-}\left(P_{k-1}\right)-\varphi_{k p}^{-}\left(P_{k}\right)\right)(1 / 2-s)
\end{aligned}
$$

where $\overline{\varphi_{k p}^{-}}\left(P_{i}\right)$ is the argument of

$$
\begin{gathered}
-a_{1 k}\left(y_{1}^{(k)}, 0\right)-a_{2 k}\left(y_{1}^{(k)}, 0\right) \lambda_{k, p+m}\left(y_{1}^{(k)}, 0\right) \text { at } P_{i}, i=k-1, k \\
-\pi<\varphi_{k p}^{-}\left(P_{i}\right)<0, \quad i=k-1, k
\end{gathered}
$$

It follows from (5.42) that

$$
\begin{aligned}
& \varphi_{k-1, p}^{+}\left(P_{k-1}\right)-\varphi_{k, p}^{+}\left(P_{k-1}\right)=\pi-\operatorname{Re} \beta_{k p}, \\
& \varphi_{k-1, p}^{-}\left(P_{k-1}\right)-\varphi_{k, p}^{-}\left(P_{k-1}\right)=\pi-\operatorname{Re} \beta_{k, p+m} .
\end{aligned}
$$

Therefore using $(6.8),\left(6.8^{\prime}\right)$ we obtain

$$
\begin{aligned}
\sum_{k=1}^{N} \frac{1}{2 \pi} \Delta \arg \prod_{p=1}^{m}\left(-a_{1 k}\left(y_{1}^{(k)}, 0\right)-a_{2 k}\left(y_{1}^{(k)}, 0\right) \lambda_{k, p+m}\left(y_{1}^{(k)}, 0\right)-i 0\right)^{1 / 2-s} \\
\quad \cdot \prod_{p=1}^{m}\left(a_{1 k}\left(y_{1}^{(k)}, 0\right)+a_{2 k}\left(y_{1}^{(k)}, 0\right) \lambda_{k p}\left(y_{1}^{(k)}, 0\right)-i 0\right)^{s-1 / 2} \\
=\frac{1}{2 \pi}\left(\frac{1}{2}-s\right) \sum_{k=1}^{N} \sum_{p=1}^{m}\left(\left(\varphi_{k p}^{-}\left(P_{k-1}\right)-\varphi_{k p}^{-}\left(P_{k}\right)\right)-\left(\varphi_{k p}^{+}\left(P_{k-1}\right)-\varphi_{k p}^{+}\left(P_{k}\right)\right)\right) \\
=\frac{1}{2 \pi}\left(\frac{1}{2}-s\right) \sum_{p=1}^{m} \sum_{k=1}^{N}\left(\left(\varphi_{k p}^{-}\left(P_{k-1}\right)-\varphi_{k-1, p}^{-}\left(P_{k-1}\right)\right)\right. \\
=\frac{1}{2 \pi}\left(\frac{1}{2}-s\right) \sum_{p=1}^{m} \sum_{k=1}^{N}\left(\left(\operatorname{Re} \beta_{k, p+m}-\pi\right)-\left(\varphi_{k p}^{+}\left(P_{k-1}\right)-\varphi_{k-1, p}^{+}\left(P_{k-1}\right)\right)\right)
\end{aligned}
$$

In (6.9) we used the notation $P_{0}=P_{N}, \varphi_{N_{p}}^{ \pm}\left(P_{N}\right)=\varphi_{0 p}^{ \pm}\left(P_{0}\right)$. 
Therefore

$$
\begin{aligned}
\sum_{k=1}^{N} & \left.\frac{1}{2 \pi} \Delta \arg \operatorname{det}\left(b_{k}^{+}\left(y_{1}^{(k)}\right)\right)^{-1} b_{k}^{-}\left(y_{1}^{(k)}\right)\right|_{y_{1}^{(k)}=a_{k}} ^{0} \\
= & \left.\sum_{k=1}^{N} \frac{1}{2 \pi} \Delta \arg \operatorname{det}\left(b_{k 0}^{+}\left(y_{1}^{(k)}\right)\right)^{-1} b_{k 0}^{-}\left(y_{1}^{(k)}\right)\right|_{y_{1}^{(k)}=a_{k}} ^{0} \\
& +\sum_{k=1}^{N} \sum_{j=1}^{m} m_{k j}+\frac{1}{2 \pi}\left(\frac{1}{2}-s\right) \sum_{k=1}^{N} \sum_{p=1}^{m}\left(\operatorname{Re} \beta_{k, p+m}-\operatorname{Re} \beta_{k, p}+2 \pi\right),
\end{aligned}
$$

where

$$
\begin{aligned}
& b_{k 0}^{+}\left(y_{1}^{(k)}\right)=\left\|B_{k j 0}\left(y_{1}^{(k)}, 0,1, \lambda_{k p}\left(y_{1}^{(k)}, 0\right)\right)\right\|_{j, p=1}^{m}, \\
& b_{k 0}^{-}\left(y_{1}^{(k)}\right)=\left\|B_{k j 0}\left(y_{1}^{(k)}, 0,-1,-\lambda_{k, p+m}\left(y_{1}^{(k)}, 0\right)\right)\right\|_{j, p=1}^{m} .
\end{aligned}
$$

Now we shall compute $\left.(1 / 2 \pi) \Delta \arg \operatorname{det} M^{(k-1)}(z)\right|_{1 / 2-i \infty} ^{1 / 2+i \infty}$. We shall assume from now on in this section that $A_{0}\left(x, \xi_{1}, \xi_{2}\right)$ is real. Then (5.37) holds. We have

$$
\begin{aligned}
& \left.\frac{1}{2 \pi} \Delta \arg \left(-1+e^{i 2 \operatorname{Re} \beta_{k j} z}\right)\right|_{1 / 2-i \infty} ^{1 / 2+i \infty} \\
& \quad=\left.\frac{1}{2 \pi} \Delta \arg \left(-1+e^{i \operatorname{Re} \beta_{k j}-2 \operatorname{Re} \beta_{k j} \tau}\right)\right|_{\tau=-\infty} ^{\infty}=\frac{1}{2 \pi}\left(\pi-\operatorname{Re} \beta_{k j}\right) .
\end{aligned}
$$

It follows from (5.34), (5.46), (5.48) that

$$
\begin{aligned}
\left.\frac{1}{2 \pi} \Delta \arg \operatorname{det} M^{(k-1)}(z)\right|_{1 / 2-i \infty} ^{1 / 2+i \infty}= & \left.\frac{1}{2 \pi} \Delta \arg \operatorname{det} M_{k-1,0}\left(z-s+\frac{1}{2}\right)\right|_{1 / 2-i \infty} ^{1 / 2+i \infty} \\
& +\left.\frac{1}{2 \pi} \Delta \arg \operatorname{det} \Delta_{k 3}(z)\right|_{1 / 2-i \infty} ^{1 / 2+i \infty} .
\end{aligned}
$$

Consider first the case of second-order elliptic operator $A_{0}(x, i \partial / \partial x)$ with real coefficients. In this case $m=1$. So $j=1, p=1, p+m=2$. We have (see (5.48))

$$
M_{k-1,0}(z)=\frac{e^{-i \pi m_{k-1,1}} b_{k-1,0}^{-}}{b_{k-1,0}^{+}}+\frac{e^{-i \pi m_{k, 1}} b_{k, 0}^{-}}{b_{k, 0}^{+}} e^{i(z-s+1 / 2)\left(2 \pi-\beta_{k 1}+\beta_{k 2}\right)},
$$

where (see (5.39), $\left(5.39^{\prime}\right)$ )

$$
\begin{gathered}
b_{k 0}^{+}=B_{k 10}\left(0,0,+1, \lambda_{k 1}\right), \quad b_{k 0}^{-}=B_{k 10}\left(0,0,-1,-\lambda_{k 2}\right), \\
b_{k-1,0}^{+}=B_{k-1,10}\left(a_{k-1}, 0,+1, \lambda_{k-1,1}\right), \\
b_{k-1,0}^{-}=B_{k-1,1,0}\left(a_{k-1}, 0,-1,-\lambda_{k-1,2}\right) .
\end{gathered}
$$

Here $B_{p 10}\left(y^{(p)}, \eta_{1}^{(p)}, \eta_{2}^{(p)}\right), p=k-1, k$ is the principal part of the boundary operator on $\Gamma_{p_{i}}$ written in $y^{(p)}$ coordinates, $(0,0)$ are the coordinates of 
the vertex $P_{k-1}$ in the $y^{(k)}$ system of coordinates, $\left(a_{k-1}, 0\right)$ are the coordinates of $P_{k-1}$ in $y^{(k-1)}$ coordinates, $\lambda_{k j}=\lambda_{k j}(0,0), j=1,2$, are roots of $A_{0}(x, \xi)=\left(\xi_{2}-\lambda_{1}(x) \xi_{1}\right)\left(\xi_{2}-\lambda_{2}(x) \xi_{1}\right)$ written in $y^{(k)}$ coordinates, and $\lambda_{k-1, j}=\lambda_{k-1, j}\left(a_{k-1}, 0\right), j=1,2$, are the roots of $A_{0}$ in $y^{(k-1)}$ coordinates. Note that (see (5.37))

$$
2 \pi+\beta_{k 1}-\beta_{k 2}=2 \operatorname{Re} \beta_{k 1}
$$

where (see (5.31))

$$
i \beta_{k j}=\ln \left(\cos \alpha_{k-1}+\lambda_{k j} \sin \alpha_{k-1}\right), \quad 0<\operatorname{Re} \beta_{k j}<2 \pi, j=1,2 .
$$

Denote

$$
\gamma_{k-1,0}=(1 / 2 \pi i) \ln \left(b_{k 0}^{-} b_{k-1,0}^{+} / b_{k 0}^{+} b_{k-1,0}^{-}\right),
$$

where we take the branch of logarithm such that

$$
-\frac{1}{2}<\operatorname{Re} \gamma_{k-1,0} \leq \frac{1}{2}, \quad k=1, \ldots, N
$$

We have

$$
\begin{aligned}
& \left.\frac{1}{2 \pi} \Delta \arg M_{k-1,0}(z)\right|_{1 / 2-i \infty} ^{1 / 2+i \infty} \\
& \quad=\left.\frac{1}{2 \pi} \Delta \arg \left(-1+e^{2 \pi i \gamma_{k-1,0}+\pi i\left(m_{k 1}+m_{k-1,1}\right)+i\left(4 \pi-2 \operatorname{Re} \beta_{k 1}\right)(z-s+1 / 2)}\right)\right|_{1 / 2-i \infty} ^{1 / 2+i \infty} .
\end{aligned}
$$

Note that the Fredholmity condition (5.47) has the following form:

$$
\operatorname{Re} \gamma_{k-1,0}+\frac{m_{k 1}+m_{k-1,1}}{2}+\left(2-\frac{\operatorname{Re} \beta_{k 1}}{\pi}\right)(1-s) \neq 0(\bmod p),
$$

where $p \in \mathbf{Z}$.

Therefore there exists an integer $p_{k-1}$ such that

$$
\operatorname{Re} \gamma_{k-1,0}+\frac{m_{k 1}+m_{k-1,1}}{2}+\left(2-\frac{\operatorname{Re} \beta_{k 1}}{\pi}\right)(1-s)=p_{k-1}+\delta_{k-1},
$$

where $0<\delta_{k-1}<1$. So

$$
\begin{aligned}
-1 & +e^{2 \pi i \gamma_{k-1,0}+\pi i\left(m_{k 1}+m_{k-1,1}\right)+i\left(4 \pi-2 \operatorname{Re} \beta_{k 1}\right)(z-s+1 / 2)} \\
& =-1+e^{2 \pi i \delta_{k-1}} e^{-2 \pi \operatorname{Im} \gamma_{k-1,0}-\left(4 \pi-2 \operatorname{Re} \beta_{k 1}\right) \tau} .
\end{aligned}
$$

Analogously to (6.12) we obtain

$$
\left.\frac{1}{2 \pi} \Delta \arg M_{k-1,0}(z)\right|_{1 / 2-i \infty} ^{1 / 2+i \infty}=\frac{1}{2 \pi}\left(\pi-2 \pi \delta_{k-1}\right) .
$$


It follows from (6.10), (6.17), (6.13), (6.12), (6.25) that the index of operator $\Phi$ in the case of a second-order operator has the following form:

$$
\text { ind } \begin{aligned}
\Phi_{s}= & \left.\sum_{k=1}^{N} \frac{1}{2 \pi} \Delta \arg \left(b_{k 0}^{+}\left(y_{1}^{(k)}\right)\right)^{-1} b_{k 0}^{-}\left(y_{1}^{(k)}\right)\right|_{y_{1}^{(k)}=a_{k}} ^{0} \\
& +\sum_{k=1}^{N} m_{k 1}+\frac{1}{2 \pi}(1 / 2-s) \sum_{k=1}^{N}\left(4 \pi-2 \operatorname{Re} \beta_{k 1}\right) \\
& +\sum_{k=1}^{N}\left(\frac{1}{2}-\delta_{k-1}\right)+\sum_{k=1}^{N}\left(\frac{1}{2}-\frac{1}{2 \pi} \operatorname{Re} \beta_{k 1}\right),
\end{aligned}
$$

where (see (6.11))

$$
\begin{aligned}
& b_{k 0}^{+}\left(y_{1}^{(k)}\right)=B_{k 10}\left(y_{r}^{(k)}, 0,1, \lambda_{k 1}\left(y_{1}^{(k)}, 0\right)\right), \\
& b_{k 0}^{-}\left(y_{1}^{(k)}\right)=B_{k 10}\left(y_{1}^{(k)}, 0,-1,-\lambda_{k 1}\left(y_{1}^{(k)}, 0\right)\right) .
\end{aligned}
$$

Note that

$$
\sum_{k=1}^{N} m_{k 1}=\sum_{k=1}^{N} \frac{m_{k 1}+m_{k-1,1}}{2}
$$

where by definition $m_{0,1}=m_{N, 1}$.

Therefore

$$
\text { ind } \begin{aligned}
\Phi_{s}= & \left.\sum_{k=1}^{N} \frac{1}{2 \pi} \Delta \arg \left(b_{k 0}^{+}\left(y_{1}^{(k)}\right)\right)^{-1} b_{k 0}^{-}\left(y_{1}^{(k)}\right)\right|_{a_{k}} ^{0} \\
& +\sum_{k=1}^{N} \frac{m_{k 1}+m_{k-1,1}}{2}+\sum_{k=1}^{N}(1-s)\left(2-\frac{\operatorname{Re} \beta_{k 1}}{\pi}\right)-\sum_{k=1}^{N} \delta_{k-1} .
\end{aligned}
$$

Substituting (6.23) into (6.29) we obtain

(6.30) ind $\Phi_{s}=\left.\sum_{k=1}^{N} \frac{1}{2 \pi} \Delta \arg \left(b_{k 0}^{+}\left(y_{1}^{(k)}\right)\right)^{-1} b_{k 0}^{-}\left(y_{1}^{(k)}\right)\right|_{a_{k}} ^{0}-\sum_{k=1}^{N} \operatorname{Re} \gamma_{k-1,0}+\sum_{k=1}^{N} p_{k-1}$,

where $p_{k-1}$ are the same as in (6.23) and $\gamma_{k-1,0}$ are the same as in (6.19).

Consider a discontinuous curve on

$$
\partial \mathscr{D}=\bigcup_{k=1}^{N} \bar{\Gamma}_{k}
$$

equal to $\left(b_{k 0}^{+}\left(y_{1}^{(k)}\right)\right)^{-1} b_{k 0}^{-}\left(y_{1}^{(k)}\right)$ on $\bar{\Gamma}_{k}$. For each vertex $P_{k-1}, 1 \leq k \leq N$,

$$
P_{0}=P_{N}, \quad P_{k-1}=\bar{\Gamma}_{k-1} \cap \bar{\Gamma}_{k},
$$

connect $\left(b_{k 0}^{+}(0)\right)^{-1} b_{k 0}^{-}(0)$ with $\left(b_{k-1,0}^{+}\left(a_{k-1}\right)\right)^{-1} b_{k-1,0}^{-}\left(a_{k-1}\right)$ by a line segment if $\left|\operatorname{Re} \gamma_{k-1,0}\right|<\frac{1}{2}$ or clockwise by an arc if $\operatorname{Re} \gamma_{k-1,0}=\frac{1}{2}$. Then we obtain a 
continuous curve in the complex plane and its winding number $\kappa_{0}$ will be an integer. We have

$$
\kappa_{0}=\left.\frac{1}{2 \pi} \sum_{k=1}^{N} \Delta \arg \left(b_{k 0}^{+}\left(y_{1}^{(0)}\right)\right)^{-1} b_{k 0}^{-}\left(y_{1}^{0}\right)\right|_{a_{k}} ^{0}-\sum_{k=1}^{N} \operatorname{Re} \gamma_{k-1,0},
$$

Note that (see (6.19))

$$
\begin{aligned}
\arg \left(b_{k-1,0}^{+}\left(a_{k-1}\right)\right)^{-1} b_{k-1,0}^{-}\left(a_{k-1}\right) & -\arg \left(b_{k}^{+}(0)\right)^{-1} b_{k-1}^{-}(0) \\
= & -2 \pi \operatorname{Re} \gamma_{k-1,0}(\bmod p), \quad p \in \mathbf{Z} .
\end{aligned}
$$

We have proved the following theorem.

Theorem 6.1. In the case of a second-order equation the index of operator $\Phi_{s}$ has the following form:

$$
\text { ind } \Phi_{s}=\kappa_{0}+\sum_{k=1}^{N} p_{k-1}
$$

where $\kappa_{0}$ is defined in (6.31), (6.27), (6.19) and $p_{k}$ are defined in (6.23).

Note that $\kappa_{0}$ is counted clockwise and is independent of $s$ and the order $m_{k 1}$ of boundary operators $B_{k 1}$ on $\Gamma_{k}$. Indeed $\kappa_{0}$ has a form analogous to the case of a boundary value problem in a smooth domain; $p_{k-1}, 1 \leq k \leq N$, gives the contribution of the vertex $P_{k-1}$ and depends on $s$ and $m_{r 1}, r=k-1, k$.

Now we shall consider the case of elliptic equations of order $2 m, m>1$. The following formula describes the dependence of

We have

$$
\left.\Delta \arg \operatorname{det} M_{k-1,0}\left(z-s+\frac{1}{2}\right)\right|_{1 / 2-i \infty} ^{1 / 2+i \infty} \text { on } s \text {. }
$$

$$
\begin{aligned}
& \left.\frac{1}{2 \pi} \Delta \arg \operatorname{det} M_{k-1,0}\left(z-s+\frac{1}{2}\right)\right|_{1 / 2-i \infty} ^{1 / 2+i \infty} \\
& \quad=\left.\frac{1}{2 \pi} \arg \operatorname{det} M_{k-1,0}(z)\right|_{-0-i \infty} ^{-0+i \infty}+m_{k-1}^{(s)}-\sum_{p=1} \frac{4 \pi-2 \operatorname{Re} \beta_{k p}}{2 \pi}(1-s),
\end{aligned}
$$

where the increment $\left.\right|_{-0-i \infty} ^{-0+i \infty}$ means that in the case when $\operatorname{det} M_{k-1,0}(z)$ has zeros on $\operatorname{Re} z=0$ we deform the contour to the negative half-plane $\operatorname{Re} z<0$ near the zeros. In (6.34) $\left|m_{k-1}^{s}\right|$ is the number of zeros of $M_{k-1,0}(z)$ including multiplicity between $\operatorname{Re} z=-\varepsilon$ and $\operatorname{Re} z=1-s ; m_{k-1}^{(s)}>0$ when $1-s>0$ and $m_{k-1}^{(s)}<0$ when $1-s<0, \varepsilon$ is small.

To prove (6.34) note that $\operatorname{det} M_{k-1,0}(z) \rightarrow \operatorname{det}\left(-b_{k-1,0}\right)$ when $\tau \rightarrow+\infty$ and $\operatorname{det} M_{k-1,0}(z) \rightarrow \operatorname{det} b_{k 0} \operatorname{det} e^{2 \pi i z} \Delta_{k 1}^{-1}(z) \Delta_{k 2}(z)$ when $\tau \rightarrow-\infty$. We have

$$
\begin{gathered}
b_{k-1,0}=\left(b_{k-1,0}^{+}\right)^{-1}\left\|e^{-i \pi m_{k-1, j}} \delta_{j p}\right\| b_{k-1,0}^{-}, \quad b_{k, 0}=\left(b_{k 0}^{+}\right)^{-1}\left\|e^{-i \pi m_{k j}} \delta_{j p}\right\| b_{k 0}^{-}, \\
\operatorname{det} e^{2 \pi i z} \Delta_{k 1}^{-1}(z) \Delta_{k 2}(z)=\exp i z \sum_{p=1}^{m}\left(4 \pi-2 \operatorname{Re} \beta_{k p}\right)
\end{gathered}
$$


Since $\arg \operatorname{det} M_{k-1,0}(z)$ changes continuously with $\operatorname{Re} z$ for large $|\tau|$, we shall connect the lines $\operatorname{Re} z=0$ and $\operatorname{Re} z=1-s$ for large $|\tau|$. The increment of $\arg \operatorname{det} M_{k-1,0}(z)$ is close to 0 for $\tau$ large and positive and $\Delta \arg \operatorname{det} M_{k-1,0}(z)$ is close to $\left(4 \pi-2 \operatorname{Re} \beta_{k p}\right)(1-s)$ when $\tau$ is large and negative. Therefore (6.34) follows from the principle of argument.

Therefore (6.10), (6.12), (6.13), (6.34) imply

$$
\text { ind } \begin{aligned}
\Phi_{s}= & \left.\sum_{k=1}^{N} \frac{1}{2 \pi} \Delta \arg \operatorname{det}\left(b_{k 0}^{+}\left(y_{1}^{(k)}\right)\right)^{-1} b_{k 0}^{-}\left(y_{1}^{(k)}\right)\right|_{y_{1}^{(k)}=a_{k}} ^{0} \\
& +\sum_{k=1}^{N}\left(\sum_{p=1}^{m} \frac{m_{k-1, p}+m_{k p}-1}{2}\right. \\
& \left.\quad+\left.\frac{1}{2 \pi} \Delta \arg \operatorname{det} M_{k-1,0}(z)\right|_{-0-i \infty} ^{-0+i \infty}+m_{k-1}^{(s)}\right) .
\end{aligned}
$$

Theorem 6.2. Index of $\Phi_{s}$ can be represented in the form (6.37) where $b_{k 0}^{ \pm}\left(y_{1}^{(k)}\right)$ are defined in (6.11), $M_{k-1,0}(z)$ is defined in (5.48), and $m_{k-1}^{(s)}$ is defined in (6.34).

Example 6.1. Consider again the case of the mixed elliptic boundary value problem, i.e., when all $\alpha_{k}=\pi$. Then (see (5.48), (5.52), (6.35))

$$
\left.\Delta \arg \operatorname{det} M_{k-1,0}(z)\right|_{-0-i \infty} ^{-0+i \infty}=\left.\Delta \arg \operatorname{det}\left(-b_{k-1,0}+e^{2 \pi i z} b_{k, 0}\right)\right|_{-0-i \infty} ^{-0+i \infty} .
$$

Let $e^{2 \pi i \sigma_{k-1, p}}, 1<p \leq m$, be the eigenvalues of $b_{k-1,0}^{-1} b_{k 0}$, where

$$
0<\operatorname{Re} \sigma_{k-1, p} \leq 1, \quad 1 \leq p \leq m, 1 \leq k \leq N
$$

Then using (6.12) we obtain

$$
\begin{aligned}
\left.\frac{1}{2 \pi} \Delta \arg \operatorname{det} M_{k-1,0}(z)\right|_{-0-i \infty} ^{-0+i \infty} & =\left.\frac{1}{2 \pi} \Delta \arg \prod_{p=1}^{m}\left(-1+e^{2 \pi i\left(z+\sigma_{k-1, p}\right)}\right)\right|_{-0-i \infty} ^{-0+i \infty} \\
& =\frac{1}{2 \pi} \sum_{p=1}^{m}\left(\pi-2 \pi \operatorname{Re} \sigma_{k-1, p}\right)
\end{aligned}
$$

Substituting (6.40) in (6.37) we obtain an expression for ind $\Phi_{s}$. The set of roots of $\operatorname{det} M_{k-1,0}(z)=0$ consists in this case of the following points:

$$
z=-\sigma_{k-1, p}+r
$$

where $1 \leq p \leq m, r \in \mathbf{Z}$.

Note that the formula (6.37) with substituted expression (6.40) is more explicit than one obtained in Example 5.1.

\section{FINITE-DIMENSIONALITY OF THE KERNEL}

In this section we shall prove the following theorem, completing the proof that operator $\mathscr{A}_{s}$ is Fredholm: 
Theorem 7.1. The kernel of operator $\mathscr{A}_{s}$ defined by (1.1) and (1.2) is finite dimensional.

Proof. We have constructed in $\S 4$ an operator $R_{s}^{(1)}$ (see (4.2)) such that

$$
\mathscr{A}_{s}^{(1)} R_{s}^{(1)}=\Phi_{s},
$$

where $\Phi_{s}$ is an operator in $\mathscr{H}_{s, N_{1}}^{(1)}=H_{s-2 m, N_{1}}(\mathscr{D}) \times \prod_{k, j} H_{0, N_{1}}\left(\Gamma_{k}\right)$ defined by (4.17), (4.27) and $\mathscr{A}_{s}^{(1)}$ is the operator defined by (1.1), (4.11). Note that $\mathscr{A}_{s}^{(1)}$ is Fredholm iff $\mathscr{A}_{s}$ is Fredholm and has the same index. It is clear that operator $\Phi_{s}$ is Fredholm iff the operator $\Phi_{s}^{(0)}$ defined by (4.27) only is Fredholm in $\mathscr{X}_{0, N_{1}}^{(2)}=\prod_{k, j} H_{0, N_{1}}\left(\Gamma_{k}\right)$. It was proved in $\S \S_{4}$ and 5 that $\Phi_{s}$ is Fredholm for all $s$ except some discrete set $\Sigma_{B}$. Therefore there exists a regularizer $\Phi_{s}^{(-1)}$ such that

$$
\begin{aligned}
& \Phi_{s} \Phi_{s}^{(-1)}=I+T_{1}, \\
& \Phi_{s}^{(-1)} \Phi_{s}=I+T_{2},
\end{aligned}
$$

where $T_{1}, T_{2}$ are compact in $\mathscr{H}_{s, N_{1}}^{(1)}, s \notin \Sigma_{B}$. Then applying $\Phi_{1}^{(-1)}$ to (7.1) from the left and from the right we obtain

$$
\begin{aligned}
& \mathscr{A}_{s}^{(1)} R_{s}^{(1)} \Phi_{s}^{(-1)}=I+T_{1}, \\
& \Phi_{s}^{(-1)} \mathscr{A}_{s}^{(1)} R_{s}^{(1)}=I+T_{2} .
\end{aligned}
$$

It follows from (7.4) that $\mathscr{A}_{s}^{(1)}$ has a closed range and finite-dimensional cokernel. To prove that $\mathscr{A}_{s}^{(1)}$ is Fredholm it is enough to prove that $\mathscr{A}_{s}^{(1)}$ has a finite-dimensional kernel. Note that (7.5) implies that $R_{s}^{(1)}$ has a closed range and a finite-dimensional kernel. We shall prove that $R_{s}^{(1)}$ is Fredholm for any $s$ and that the index of $R_{s}^{(1)}$ is equal to zero. Then it will follow from (7.1) that $\mathscr{A}_{s}^{(1)}$ is Fredholm when $\Phi_{s}$ is Fredholm and

$$
\text { ind } \mathscr{A}_{s}^{(1)}=\operatorname{ind} \Phi_{s}-\text { ind } R_{s}^{(1)}=\text { ind } \Phi_{s} \text {. }
$$

Proposition 7.1. Operator $R_{r}^{(1)}$ is Fredholm iff $R_{s}^{(1)}$ is Fredholm for some $s$ and ind $R_{r}^{(1)}=$ ind $R_{s}^{(1)}, \forall r$.

Proof. Changing lower-order terms of $A(x, i \partial / \partial x)$ we shall find an operator $A^{(-1)}$ from $H_{s-2 m, N_{1}}(\mathscr{D})$ to $H_{s, N_{1}}(\mathscr{D})$ that gives a particular solution of $(1.1)$ :

$$
p_{\mathscr{D}} A(x, D) A^{(-1)} f=f \text {. }
$$

Note that $A^{(-1)}$ coincides modulo a lower-order operator with $A_{0}^{(-1)}(x, i \partial / \partial x)$ (see (4.2)). Denote $H_{A}^{s}(\mathscr{D})=\operatorname{ker} A \cap H_{s, N_{1}}(\mathscr{D})$ where $\operatorname{ker} A$ is the space of 
all distribution solutions of $A(x, i \partial / \partial x) u=0$ in $\mathscr{D}$. Denote by $R_{s}^{(0)}$ the following operator from $\mathscr{H}_{0, N_{1}}^{(2)}$ to $H_{A}^{s}(\mathscr{D})$ :

$$
R_{s}^{(0)} c=p_{\mathscr{D}} \sum_{k=1}^{N} \sum_{j=1}^{m} \psi_{k} R_{k j} c_{k j}-A^{(-1)} \sum_{k, j} T_{k j} c_{k j},
$$

where $A^{(-1)}$ is the same as in (7.7) and $T_{k j}$ are the same as in (4.16). Note that $R_{s}^{(1)}(g, c)$ and $A^{(-1)} g+R_{s}^{(0)} c$ differ only in the lower-order terms. Therefore $R_{s}^{(1)}$ is Fredholm iff $A^{(-1)} g+R_{s}^{(0)} c$ is Fredholm and

$$
\text { ind } R_{s}^{(1)}=\operatorname{ind}\left(A^{(-1)} g+R_{s}^{(0)} c\right) \text {. }
$$

Also the equation

$$
u=A^{(-1)} g+R_{s}^{(0)} c
$$

is equivalent to the equations

$$
\begin{gathered}
g=A u, \\
u-A^{(-1)}(A u)=R_{s}^{(0)} c,
\end{gathered}
$$

where we used that $A R_{s}^{(0)}=0$. Therefore $A^{(-1)} g+R_{s}^{(0)} c$ is Fredholm iff $R_{s}^{(0)}$ is Fredholm and

$$
\text { ind } R_{s}^{(0)}=\operatorname{ind}\left(A^{(-1)} g+R_{s}^{(0)} c\right)=\operatorname{ind} R_{s}^{(1)} .
$$

Let $\Lambda_{-, \tau}^{s(x)}$ be a pseudodifferential operator with $\left(a_{1}(x) \xi_{1}+a_{2}(x) \xi_{2}+i \tau\right)^{s(x)}$ (see (4.4)) where $\tau>0$ is large. If $u \in H_{A}^{r}(\mathscr{D})$ then $\Lambda_{-, \tau}^{s(x)} u \in H_{r-s}(\mathscr{D})$. Indeed this is obvious if $s \geq 0$. Inside $\mathscr{D} \Lambda_{-, \tau}^{s(x)} u \in C^{\infty}$ since $A u=0$. If $s<0$ then it is clear that $\Lambda_{-, \tau}^{s(x)} u$ has a smoothness of order $r-s$ in the direction of the vector field $\left(a_{1}(x), a_{2}(x)\right)$. The smoothness in the direction transversel to $\left(a_{1}(x), a_{2}(x)\right)$ follows from the ellipticity of $A$ and since $A u=0$ in $\mathscr{D}$ (using the partition of unity and constructing "minus"-operator $E_{1}, E_{2}$ locally one can apply a proof analogous to (3.19), (3.20), (3.21)). We have

$$
\Lambda_{-, \tau}^{-s(x)} \Lambda_{-, \tau}^{s(x)}=I+K_{0},
$$

where $K_{0}$ is an operator of zero order with a small norm if $\tau$ is large.

Since $\Lambda_{-\tau}^{s(x)}$ is a "minus"-operator we have using (7.13) and that $u \in H_{A}^{r}(\mathscr{D})$

$$
p_{\mathscr{D}} A p_{\mathscr{D}} \Lambda_{-\tau}^{s(x)} u=p_{\mathscr{D}} \Lambda_{-\tau}^{s(x)} \cdot p_{\mathscr{D}} A u+p_{\mathscr{D}} K_{2 m} \Lambda_{-\tau}^{s(x)} u=p_{\mathscr{D}} K_{2 m} \Lambda_{-\tau}^{s(x)} u,
$$

where $K_{2 m}$ is an operator of order $2 m$ with a small norm when $\tau$ is large. Therefore the operator

$$
C_{s}=p_{\mathscr{D}} \Lambda_{-, \tau}^{s(x)}-A^{(-1)} p_{\mathscr{D}} K_{2 m} \Lambda_{-, \tau}^{s(x)}
$$


maps $H_{A}^{r}(\mathscr{D})$ to $H_{A}^{r-s}(\mathscr{D})$ since $A\left(p_{\mathscr{D}} \Lambda_{-, \tau}^{s(x)}-A^{(-1)} p_{\mathscr{D}} K_{2 m} \Lambda_{-\tau}^{s(x)}\right) u=0$ in $\mathscr{D}$ for any $u \in H_{A}^{r}(\mathscr{D})$. Analogously we can construct an operator $C_{-s}=p_{\mathscr{D}} \Lambda_{-, \tau}^{-s(x)}-$ $A^{(-1)} K_{2 m}^{(1)} \Lambda_{-, \tau}^{-s(x)}$ that maps $H_{A}^{r_{1}}(\mathscr{D})$ to $H_{A}^{r_{1}+s}(\mathscr{D})$ for any $r_{1}$. We have

$$
\begin{aligned}
& C_{s} C_{-s}=I+K_{01}, \\
& C_{-s} C_{s}=I+K_{02},
\end{aligned}
$$

where $K_{01}, K_{02}$ are operators in $H_{A}^{r}(\mathscr{D})$ with a small norm since $\tau$ is large. It follows from $(7.16),(7.17)$ that $C_{s}$ maps $H_{A}^{r}(\mathscr{D})$ onto $H_{A}^{r-s}(\mathscr{D})$ and in particular ind $C_{s}=0$. It follows from (4.5) that

$$
\Lambda_{-, \tau}^{s(x)} \cdot R_{r}^{(0)} c=R_{r-s}^{(0)} c+T_{3} c
$$

where ord $T_{3} \leq$ ord $R_{r-s}^{(0)}-1$.

Note that operator $K_{2 m}$ restricted to $H_{A}^{r_{1}}(\mathscr{D})$ is of order $2 m-1$. Indeed $K_{2 m}$ can be represented in the form

$$
K_{2 m}=K_{2 m}^{(0)} \Lambda_{-, \tau}^{-1},
$$

where ord $K_{2 m}^{(0)}=2 m$, and ord $\Lambda_{-, \tau}^{(-1)}=-1$ as an operator on $H_{A}^{r_{1}}(\mathscr{D})$.

Therefore we have

$$
C_{s} R_{r}^{(0)}=R_{r-s}^{(0)}+T_{4}
$$

where ord $T_{4} \leq$ ord $R_{r-s}^{(0)}-1$.

It follows from (7.19) that $R_{r}^{(0)}$ is Fredholm iff $R_{r-s}^{(0)}$ is Fredholm and ind $R_{r}^{(0)}=$ ind $R_{r-s}^{(0)}$, that is, the index of $R_{r}^{(0)}$ is independent of $r$, assuming that we can prove that $R_{s}^{(0)}$ is Fredholm for some $s$.

Proposition 7.2. $R_{s}^{(0)}$ has a finite-dimensional kernel and closed range for all $s$. (We shall call such an operator semi-Fredholm.)

Proof. Note that (7.1) is equivalent to the equality

$$
\mathscr{A}_{s}^{(0)} R_{s}^{(0)}=\Phi_{s}^{(0)} \text {, }
$$

where $\mathscr{A}_{s}^{(0)}$ is an operator from $H_{A}^{s}(\mathscr{D})$ to $\mathscr{H}_{0, N_{1}}^{(s)}$ defined by the left-hand side of (4.11) and $\Phi_{s}^{(0)}$ has the same principal part as the left-hand side of (4.27). From (7.20) we have that $R_{s}^{(0)}$ is semi-Fredholm for any $s \notin \Sigma_{B}$ when $\Sigma_{B}$ is the exceptional set for $\mathscr{A}_{s}^{(0)}$. Changing the boundary conditions $B_{k j}$ to $B_{k j} \Lambda_{-, k}^{\delta}$ we obtain an exceptional set of the form $\Sigma_{B}+\delta$. Choosing $\delta>0$ such that $\left(\Sigma_{B}+\delta\right) \cap \Sigma_{B}=\varnothing$ we obtain that $R_{s}^{(0)}$ is semi-Fredholm for any $s$.

Also $R_{s}^{(0)}$ is semi-Fredholm for any domain $\mathscr{D}$ and for any properly elliptic operator $A(x, i \partial / \partial x)$ and the norm of $R_{s}^{(0)}$ depends continuously on the coefficients of $A(x, i \partial / \partial x)$ and on the domain $\mathscr{D}$. 
Proposition 7.3. If $R_{s}^{(0)}$ is Fredholm for some domain $\mathscr{D}_{0}$ and for some elliptic operator $A^{(0)}(x, i \partial / \partial x)$ then $R_{s}^{(0)}$ will be Fredholm for any properly elliptic $A(x, i \partial / \partial x)$ and any domain $\mathscr{D}$ that can be obtained by a deformation of $\mathscr{D}_{0}$ and $A^{(0)}(x, i \partial / \partial x)$.

Proof. Let $A^{(t)}(x, i \partial / \partial x)$ and $\mathscr{D}_{t}$ be a deformation such that $0 \leq t \leq 1$, $A^{(1)}(x, i \partial / \partial x)=A\left(x, \partial / \partial x\right.$.) , and $\mathscr{D}_{1}=\mathscr{D}$. Since the set of all $t$ such that $R_{s}^{(0)}(t)$ is Fredholm is open we have that $R_{s}^{(0)}(t)$ is Fredholm for small $t$. Let $t_{0}$ be such that $R_{s}^{(0)}\left(t_{0}\right)$ is semi-Fredholm but it is not Fredholm and $R_{s}^{(0)}(t)$ is Fredholm for $t<t_{0}$. We have $\operatorname{dim} \operatorname{coker} R_{s}^{(0)}\left(t_{0}\right)=+\infty$. Since the range of $R_{s}^{(0)}\left(t_{0}\right)$ is closed there exists a bounded operator $A\left(t_{0}\right)$ such that

$$
A\left(t_{0}\right) R_{s}^{(0)}\left(t_{0}\right)=I+T_{5},
$$

where $T_{5}$ is compact. Note that $\operatorname{dim} \operatorname{ker} A\left(t_{0}\right)=+\infty$. We have for $t<t_{0}$, $\left|t-t_{0}\right|$ small:

$$
A\left(t_{0}\right) R_{s}^{(0)}(t)=A\left(t_{0}\right) R_{s}^{(0)}\left(t_{0}\right)+A\left(t_{0}\right)\left(R_{s}^{(0)}(t)-R_{s}^{(0)}\left(t_{0}\right)\right)=I+K_{3}+T_{5},
$$

where $K_{3}=A\left(t_{0}\right)\left(R_{s}^{(0)}(t)-R_{s}^{(0)}\left(t_{0}\right)\right)$ has a small norm, $\left\|K_{3}\right\|<1$ when $\left|t-t_{0}\right|$ is small. Since $R_{s}^{(0)}(t), t<t_{0}$, and $I+K_{3}+T_{5}$ are Fredholm, (7.22) implies that $A\left(t_{0}\right)$ must be Fredholm and this contradicts the fact that dim ker $A\left(t_{0}\right)=+\infty$. Therefore $R_{s}^{(0)}(t)$ must be Fredholm for all $t \in[0,1]$.

To prove that $R_{s}^{(0)}$ are Fredholm operators and to compute the index of $R_{s}^{(0)}$ consider the case when $\mathscr{D}$ is deformed to a smooth domain $\mathscr{D}_{0}$; that is, all angles $\alpha_{k}=\pi, k=1, \ldots, N$, and $A^{(0)}(i \partial / \partial x)$ is an elliptic operator with constant coefficients and a positive symbol $A^{(0)}(\xi)>C\left(|\xi|^{2 m}+1\right)$. Take $s=m+\varepsilon, 0<\varepsilon<\frac{1}{2}$, and consider the Dirichlet boundary value problem

$$
\begin{gathered}
A^{(0)}(i \partial / \partial x) u=0 \quad \text { in } \mathscr{D}_{0}, \\
\partial^{j-1} u /\left.\partial n^{j-1}\right|_{\Gamma_{k}}=h_{k j}, \quad 1 \leq k \leq N, 1 \leq j \leq m,
\end{gathered}
$$

where $\partial / \partial n$ is the normal derivative to $\partial \mathscr{D}_{0}$.

Proposition 7.4. Index of the boundary value problem (7.23), (7.24) in $H_{m+\varepsilon}\left(\mathscr{D}_{0}\right)$ is equal to $-(m(m+1) / 2) N$.

Proof. If $u \in H_{m+\varepsilon}\left(\mathscr{D}_{0}\right)$ satisfies (7.24) with $h_{k j}=0$ then $u \in \stackrel{\circ}{H}_{m+\varepsilon}\left(\mathscr{D}_{0}\right)$ since $0<\varepsilon<\frac{1}{2}$. Here $\stackrel{\circ}{H}_{m+\varepsilon}\left(\mathscr{D}_{0}\right)$ is the subspace of $H_{m+\varepsilon}\left(\mathbf{R}^{2}\right)$ of functions with supports in $\overline{\mathscr{D}}_{0}$. Then the integration by parts gives that $u=0$ if $u$ satisfies (7.23) and $u \in \stackrel{\circ}{H}_{m+\varepsilon}\left(\mathscr{D}_{0}\right)$. Therefore $\operatorname{ker} \mathscr{A}_{m+\varepsilon}^{(0)}=0$ where $\mathscr{A}_{m+\varepsilon}^{(0)}$ is the operator from $\operatorname{ker} A^{(0)} \cap H_{m+\varepsilon}\left(\mathscr{D}_{0}\right)$ to $\mathscr{H}_{m+\varepsilon}=\prod_{k, j} H_{m+\varepsilon-j+1 / 2}\left(\Gamma_{k}\right)$. We also compute also the cokernel of $\mathscr{A}_{m+\varepsilon}^{(0)}$. If $\left\{h_{k j}\right\}$ belong to the range of $\mathscr{A}_{m+\varepsilon}^{(0)}$ then $\left\{h_{k j}\right\}$ 
must satisfy the compatibility conditions at the vertices $P_{k}, k=1, \ldots, N$. Indeed $h_{k-1,1}$ and $h_{k, 1}$ must have equal values at $P_{k-1}=\bar{\Gamma}_{k-1} \cap \bar{\Gamma}_{k}$, and the same for the tangential derivatives of $h_{k-1,1}$ and $h_{k, 1}$ up to the order $m-1$. Analogously $h_{k-1, j}$ and $h_{k, j}$ must satisfy $m-j+1$ compatibility conditions at each $P_{k-1}, 1 \leq k \leq N$, since they are traces of

$$
\partial^{j-1} u /\left.\partial n^{j-1}\right|_{\partial \mathscr{D}_{0}} \in H_{m+\varepsilon-j+1 / 2}\left(\partial \mathscr{D}_{0}\right) \text {. }
$$

Therefore we have $(m+m-1+\cdots+1) N=(m(m+1) / 2) N$ compatibility conditions. If $\left\{h_{k j}\right\}$ satisfy these compatibility conditions, then there exists a function $v \in H_{m+\varepsilon}\left(\mathscr{D}_{0}\right)$ such that

$$
\partial^{j-1} v /\left.\partial n^{j-1}\right|_{\Gamma_{k}}=h_{k j}, \quad 1 \leq k \leq N, 1 \leq j \leq m .
$$

Denote by $w \in \stackrel{\circ}{H}_{m+\varepsilon}(\mathscr{D})$ the solution of the equation

$$
A^{(0)}(i \partial / \partial x) w=-A^{(0)}(i \partial / \partial x) v,
$$

where $-A^{(0)}(i \partial / \partial x) v \in H_{-m+\varepsilon}\left(\mathscr{D}_{0}\right)$ is known. Such a solution $w$ exists and it is unique since $0<\varepsilon<\frac{1}{2}$ (see, for example, Example 17.1 in [3]). Then $u=v+w \in H_{m+\varepsilon}\left(\mathscr{D}_{0}\right)$ will be the solution of (7.23), (7.24). Therefore we computed that ind $\mathscr{A}_{m+\varepsilon}^{(0)}=-(m(m+1) / 2) N$.

Now compute ind $\Phi_{m+\varepsilon}^{(0)}$. In the case of the Dirichlet boundary conditions we have

$$
\begin{gathered}
b_{k 0}^{+}\left(y_{1}^{(k)}\right)=\left\|\lambda_{k p}^{j-1}\left(y_{1}^{(k)}, 0\right)\right\|_{j, p=1}^{m}, \\
b_{k 0}^{-}\left(y_{1}^{(k)}\right)=\left\|\left(-\lambda_{k, p+m}\left(y_{1}^{(k)}, 0\right)\right)^{j-1}\right\|_{j, p=1}^{m} .
\end{gathered}
$$

Since the boundary is smooth and the boundary conditions are the same on each $\Gamma_{k}$ we have $b_{k-1,0}^{+}=b_{k, 0}^{+}, b_{k-1,0}^{-}=b_{k, 0}^{-}$(see (5.48)), and therefore $b_{k-1,0}=b_{k, 0}(\operatorname{see}(6.38))$. Then $\sigma_{k-1, p}=1,1 \leq k \leq N, 1 \leq p \leq m$ (see (6.39)). Since (7.27), (7.28) are the Vandermonde matrices we have

$$
\begin{aligned}
& \operatorname{det}\left(b_{k 0}^{+}\left(y_{1}^{(k)}\right)\right)^{-1} b_{k, 0}^{-}\left(y_{1}^{(k)}\right) \\
& \quad=\prod_{r<p}\left(-\lambda_{k, r+m}\left(y_{1}^{(k)}, 0\right)+\lambda_{k, p+m}\left(y_{1}^{(k)}, 0\right)\right)\left(\lambda_{k, r}\left(y_{1}^{(k)}, 0\right)-\lambda_{k, p}\left(y_{1}^{(k)}, 0\right)\right)^{-1} .
\end{aligned}
$$

Note that

$$
\operatorname{det}\left(b_{k 0}^{+}\left(y_{1}^{(k)}\right)\right)^{-1} b_{k, 0}^{-}\left(y_{1}^{(k)}\right), \quad k=1, \ldots, N,
$$

are parts of a continuous function on $\partial \mathscr{D}_{0}$ since boundary conditions are the same on each $\Gamma_{k}, 1 \leq k \leq N_{1}$. Since $\operatorname{Im} \lambda_{k p}\left(y_{1}^{(k)}, 0\right)<0$ for all $y_{1}^{(k)}, 1 \leq k \leq$ $N$, we have

$$
-\pi<\arg \left(\lambda_{k p}\left(y_{1}^{(k)}, 0\right)-\lambda_{k, r}\left(y_{1}^{(k)}, 0\right)\right)<\pi
$$


for all $y_{1}^{(k)}$. Also

$$
-\pi<\arg \left(\lambda_{k, p+m}\left(y_{1}^{(k)}, 0\right)-\lambda_{k, r+m}\left(y_{1}^{(k)}, 0\right)\right)<\pi .
$$

Therefore

$$
\left.\frac{1}{2 \pi} \sum_{k=1}^{N} \Delta \arg \operatorname{det}\left(b_{k}^{+}\left(y_{1}^{(k)}\right)\right)^{-1} b_{k}^{-}\left(y_{1}^{(k)}\right)\right|_{y_{1}^{(k)}=a_{k}} ^{0}=0 .
$$

We have that $\operatorname{det} M_{k-1,0}(z)=\left(-1+e^{2 \pi i z}\right)^{m} \operatorname{det} b_{k 0}$. So $\operatorname{det} M_{k-1,0}(z)=0$ has a root of multiplicity $m$ at $z=p, p \in \mathbf{Z}$. Therefore formulas (6.37) and (6.40) give

$$
\text { ind } \begin{aligned}
\Phi_{m+\varepsilon}^{(0)} & =\sum_{k=1}^{N}\left(\sum_{p=1}^{m} \frac{p-1+p-1-1}{2}+\frac{1}{2 \pi} \sum_{p=1}^{m}(\pi-2 \pi)-m(m-1)\right) \\
& =N\left(\frac{m(m-1)}{2}-m-m(m-1)\right)=-\frac{m(m+1)}{2} N .
\end{aligned}
$$

Therefore $R_{s}^{(0)}$ is a Fredholm operator when $N_{1}=0$ and

$$
\text { ind } R_{s}^{(0)}=\text { ind } R_{m+\varepsilon}^{(0)}=\text { ind } \Phi_{m+\varepsilon}^{(0)}-\text { ind } \mathscr{A}_{m+\varepsilon}^{(0)}=0 \text {. }
$$

Proposition 7.5. Operator $R_{s}^{(1)}$ is Fredholm of index zero for any $N_{1} \geq 0$.

Proof. Since $\operatorname{ker} \mathscr{A}_{m+\varepsilon}^{(1)}=0$ for any $N_{1} \geq 0$ we have that $R_{s}^{(0)}$ and $R_{s}^{(1)}$ are Fredholm for any $N_{1} \geq 0$. We shall show that ind $R_{s}^{(0)}=$ ind $R_{s}^{(1)}=0$ for any $N_{1} \geq 0$. We have that $R_{s}^{(1)}$ is bounded from $\mathscr{H}_{s, N_{1}}$ to $H_{s, N_{1}}(\mathscr{D})$ for $N_{1} \geq 0$. The following regularity property holds:

$$
\begin{aligned}
& \text { If } u \in R_{s}^{(1)} F \text { and } F \in \mathscr{H}_{s, N_{1}}, u \in H_{s, N_{1}+N_{2}}(\mathscr{D}), N_{2}>0 \text {, then } \\
& \text { also } F \in \mathscr{H}_{s, N_{1}+N_{2}} .
\end{aligned}
$$

Indeed, applying $\mathscr{A}_{S}^{(1)}$ we obtain

$$
\mathscr{A}_{s}^{(1)} u=\mathscr{A}_{s}^{(1)} R_{s}^{(1)} F=\Phi_{s}^{(1)} F \text {, i.e., } \Phi_{s}^{(1)} F=\mathscr{A}_{s}^{(1)} u \text {. }
$$

Since $\mathscr{A}_{s}^{(1)}$ is bounded from $H_{s, N_{1}+N_{2}}(\mathscr{D})$ to $\mathscr{H}_{s, N_{1}+N_{2}}$ we have that $\Phi_{s}^{(1)} F \in$ $\mathscr{H}_{s, N_{1}+N_{2}}$. Applying the regularizer of $\Phi_{s}^{(1)}$ we obtain

$$
\Phi_{s}^{(-1)} \mathscr{A}_{s}^{(1)} u=F+T_{-1} F
$$

where $T_{-1}$ is bounded from $\mathscr{H}_{s, N_{1}+1}$ to $\mathscr{H}_{s, N_{1}}$. Therefore $F \in \mathscr{H}_{s, N_{1}+1}$. Repeatedly applying $\Phi_{s}^{(-1)}$ we finally obtain that $F \in \mathscr{H}_{s, N_{1}+N_{2}}$. Therefore $\operatorname{ker} R_{s}^{(1)} \in \mathscr{H}_{s, N}, \forall N \geq 0$. Also coker $R_{s}^{(1)} \in\left(\mathscr{H}_{s, N}\right)^{*}$ for any $N \geq 0$. Indeed let $F_{1}^{*}, \ldots, F_{n}^{*}$ be the basis of $\operatorname{coker} R_{s}^{(1)}$ in $\left(\mathscr{H}_{s, 0}\right)^{*}$. If $u \in H_{s, N}(\mathscr{D})$ and $\left(u, F_{k}^{*}\right)=0,1 \leq k \leq n$, then there exists $F \in \mathscr{H}_{s, 0}$ such that $u=R_{s}^{(1)} F$ 
since $H_{s, N}(\mathscr{D}) \subset H_{s, 0}(\mathscr{D})$. Then (7.33) implies that $F \in \mathscr{H}_{s, N}$, that is, $u \in \operatorname{Im} R_{s, N}^{(1)}$ where $R_{s, N}^{(1)}$ is the restriction of the operator $R_{s}^{(1)}$ to $\mathscr{H}_{s, N} \subset \mathscr{H}_{s, 0}$. So coker $R_{s, N}^{(1)} \subset \operatorname{coker} R_{s, 0}^{(1)}$. The inclusion coker $R_{s, 0}^{(1)} \subset \operatorname{coker} R_{s, N}^{(1)}$ holds since $\operatorname{Im} R_{s, N}^{(1)} \subset \operatorname{Im} R_{s, 0}^{(1)}$. Therefore $\operatorname{coker} R_{s, N}^{(1)}=\operatorname{coker} R_{s, 0}^{(1)}$ for all $N \geq 0$. So ind $R_{s, N}^{(1)}=$ ind $R_{s, 0}^{(1)}$ for all $N \geq 0$.

Using the regularizer $\Phi_{s}^{(-1)}$ one can show analogously that the regularity in $N$ holds for $\Phi_{s}^{(1)}$ and therefore ind $\Phi_{s, N}^{(1)}=\operatorname{ind} \Phi_{s}^{(1)}$ for all $N \geq 0$. Therefore ind $\mathscr{A}_{s, N_{1}}=$ ind $\mathscr{A}_{s, N_{1}+N_{2}}$ for all $N_{2} \geq 0$ where $N_{1} \geq 0$ is the minimal integer such that $N_{1}>m_{k j}+\frac{1}{2}-s$.

Summarizing the results of $\S \S 4,5,7$, we get the following theorem.

Theorem 7.2. Operator $\mathscr{A}_{s}$ defined by the left-hand sides of (1.1), (1.2) is a Fredholm operator from $H_{s, N_{1}}(\mathscr{D})$ to $\mathscr{H}_{s, N_{1}}$ iff(1.7) and (5.49) are satisfied for all $k=1, \ldots, N$. The index of $\mathscr{A}_{s}$ is given by the formula (5.50). In the case of real-valued symbol $A_{0}(x, \xi)$ one can use the formula (6.37) for $m>1$ and (6.33) for the case of a second-order operator.

\section{EXAMPLES AND REMARKS}

In this section we shall consider some boundary value problems for the Laplacian

$$
\Delta u=f, \quad x \in \mathscr{D} .
$$

Example 8.1. The Dirichlet boundary value problem. Consider the Dirichlet boundary conditions

$$
\left.u\right|_{\Gamma_{k}}=h_{k}, \quad 1 \leq k \leq N .
$$

In the case of the Laplacian we have (see (6.17), (6.18)):

$$
\begin{gathered}
\lambda_{k 1}=-i, \quad \lambda_{k 2}=i, \\
\beta_{k 1}=(1 / i) \ln \left(\cos \alpha_{k-1}-i \sin \alpha_{k-1}\right)=2 \pi-\alpha_{k-1}, \\
\beta_{k 2}=(1 / i) \ln \left(\cos \alpha_{k-1}+i \sin \alpha_{k-1}\right)=\alpha_{k-1}, \\
\beta_{k 1}+2 \pi-\beta_{k 2}=2 \beta_{k 1}=2\left(2 \pi-\alpha_{k-1}\right),
\end{gathered}
$$

where $\alpha_{k-1}$ are the interior angles at $P_{k-1}, 1 \leq k \leq N, P_{0}=P_{N}$. For the case of the Dirichlet boundary conditions we obtain (see (6.14), (6.15), (6.16), (6.19))

$$
\begin{gathered}
b_{k 0}^{ \pm}\left(y_{1}^{(k)}\right)=1, \quad b_{k 0}^{ \pm}=1, \quad m_{k, 1}=0, \\
M_{k-1,0}(z)=-1+e^{-i(z-s+1 / 2) 2 \alpha_{k-1}}, \\
\gamma_{k-1,0}=0, \quad 1 \leq k \leq N .
\end{gathered}
$$


Therefore the Fredholmity condition (6.22) has the following form:

$$
s \neq 1+p \pi / \alpha_{k-1}, \quad 1 \leq k \leq N, p \in \mathbf{Z} .
$$

We shall also require that (see (1.16))

$$
s \neq 0,-1,-2, \ldots,
$$

If $f=0$ in $(8.1)$ then the restriction $\left(8.8^{\prime}\right)$ is not needed. Let $p_{k-1}$ be such that (see (6.23))

$$
\left(\alpha_{k-1} / \pi\right)(1-s)=p_{k-1}+\delta_{k-1},
$$

where $p_{k-1}$ is an integer and $0<\delta_{k-1}<1$. Then the index $\kappa$ of the Dirichlet problem is given by the formula (see (6.33))

$$
\kappa=\sum_{k=1}^{N} p_{k-1}
$$

where $p_{k-1}$ are defined by (8.9).

In particular, $\kappa=0$ iff

$$
1-\pi / \alpha_{k-1}<s<1, \quad 1 \leq k \leq N .
$$

Note that $\kappa=-N$ iff $1<s<1+\pi / \alpha_{k-1}, 1 \leq k \leq N$. This result is easy to explain. If $u \in H_{s}(\mathscr{D}), s>1$, then the restrictions to $\Gamma_{k}$ belong to $H_{s-1 / 2}\left(\Gamma_{k}\right)$ and also the restrictions to the vertices $P_{k-1}, 1 \leq k \leq N$, exist. Therefore $h_{k-1,1}$ and $h_{k, 1}$ have the same value at $P_{k-1}$ :

$$
\left.h_{k, 1}\right|_{P_{k-1}}=\left.h_{k-1,1}\right|_{P_{k-1}}, \quad 1 \leq k \leq N .
$$

These $N$ compatibility conditions imply that $\kappa=-N$. Also note that the boundary value problem $(8.1),(8.2)$ is not Fredholm in $H_{1}(\mathscr{D})$. This happens because we consider the Dirichlet boundary value problem with nonhomogeneous boundary conditions (8.2) and $s=1$ is the critical value for the problem of the restriction to the vertices. If one included compatibility conditions in the class of admissible right-hand sides, in particular if one considered the Dirichlet problem with zero boundary conditions, then it would also be Fredholm for $s=1$ and the index for $s>1$ would differ from (8.10) by the number of compatibility conditions. We shall consider the case of a homogeneous boundary value problem in detail elsewhere.

Example 8.2. The Neumann problem. Consider the Neumann boundary conditions

$$
\partial u /\left.\partial n_{k}\right|_{\Gamma_{k}}=h_{k 1}, \quad 1 \leq k \leq N,
$$

where $n_{k}$ is the normal to $\Gamma_{k}$. In this case $m_{k 1}=1, b_{k 0}^{ \pm}\left(y_{1}^{(k)}\right)=-1, \gamma_{k 0}=0$. The Fredholmity condition has the following form (see (6.22)):

$$
s \neq 1+p \pi / \alpha_{k-1}, \quad 1 \leq k \leq N, p \in \mathbf{Z} .
$$


Also we require that (see (1.16)) $\left(8.8^{\prime}\right)$ holds if $f \neq 0$ in (8.1). The index of the Neumann problem $(8.1),(8.13)$ is given by the formula $(8.10)$ where (see (6.23))

$$
1+\left(\alpha_{k-1} / \pi\right)(1-s)=p_{k-1}+\delta_{k-1}, \quad p_{k-1} \text { are integers, } 0<\delta_{k-1}<1
$$

In particular, $\kappa=0$ iff

$$
1<s<1+\pi / \alpha_{k-1}, \quad 1 \leq k \leq N
$$

Example 8.3. The Dirichlet-Neumann boundary value problem. Consider the boundary value problem of the form

$$
\left.u\right|_{\Gamma_{k_{j}}}=h_{k_{j}},\left.\quad \frac{\partial u}{\partial n_{k_{r}}}\right|_{\Gamma_{k_{r}}}=h_{k_{r}}, \quad 1 \leq j \leq p, 1 \leq r \leq N-p ;
$$

that is, on some $\Gamma_{k}$ we have the Dirichlet boundary conditions and on some $\Gamma_{k}$ we have the Neumann boundary conditions. Again in this case the formula for the index has the form (8.10) where $p_{k-1}$ is the contribution of the vertex $P_{k-1}, 1 \leq k \leq N$. Since each $p_{k-1}$ depends only on the boundary conditions on $\Gamma_{k-1}$ and $\Gamma_{k}$ that are joined by the vertex $P_{k-1}$, it is enough to consider the case when we have the Dirichlet boundary condition on $\Gamma_{k-1}$ and the Neumann boundary condition on $\Gamma_{k}$. In this case $m_{k-1,1}=0, m_{k, 1}=1, b_{k-1,0}^{ \pm}=1$, $b_{k, 0}^{ \pm}=-1, \gamma_{k 0}=0$. Therefore the Fredholmity condition has the form (see (6.22)):

$$
s \neq 1+\pi / 2 \alpha_{k-1}+\left(\pi / \alpha_{k-1}\right) p, \quad p \in \mathbf{Z},
$$

and $p_{k-1}$ is given by the formula

$$
\frac{1}{2}+\left(\alpha_{k-1} / \pi\right)(1-s)=p_{k-1}+\delta_{k-1},
$$

$0<\delta_{k-1}<1, p_{k-1}$ is an integer. In particular, $p_{k-1}=0$ iff

$$
1-\pi / 2 \alpha_{k-1}<s<1+\pi / 2 \alpha_{k-1} \text {. }
$$

Example 8.4. Oblique derivative problem. Consider the following boundary condition:

$$
-c_{1 k}\left(y_{1}^{(k)}\right) \frac{\partial u}{\partial n_{k}}+\left.c_{2 k}\left(y_{1}^{(k)}\right) \frac{\partial u}{\partial \tau_{k}}\right|_{\Gamma_{k}}=h_{k 1}, \quad 1 \leq k \leq N,
$$

where $\partial / \partial n_{k}$ is the normal derivative and $\partial / \partial \tau_{k}$ is the tangential derivative to $\Gamma_{k}, c_{1 k}^{2}+c_{2 k}^{2}>0$ on $\bar{\Gamma}_{k}$. In this case we have (see (6.27))

$$
\begin{aligned}
& b_{k 0}^{+}\left(y_{1}^{(k)}\right)=c_{1 k}\left(y_{1}^{(k)}\right)-i c_{2 k}\left(y_{1}^{(k)}\right), \\
& b_{k 0}^{-}\left(y_{1}^{(k)}\right)=c_{1 k}\left(y_{1}^{(k)}\right)+i c_{2 k}\left(y_{1}^{(k)}\right) .
\end{aligned}
$$

Note that $b_{k 0}^{+}=\overline{b_{k 0}^{-}}$. Denote

$$
\gamma_{k-1,0}^{(1)}=(1 / 2 \pi i) \ln \left(b_{k 0}^{-}(0) / b_{k-1,0}^{-}\left(a_{k-1}\right)\right),
$$


where we choose the branch of logarithm such that

$$
-\frac{1}{2}<\operatorname{Re} \gamma_{k-1,0}^{(1)} \leq \frac{1}{2} \text {. }
$$

Note that $\operatorname{Re} \gamma_{k-1,0}=2 \operatorname{Re} \gamma_{k-1,0}^{(1)}(\bmod p)$ where $\gamma_{k-1,0}$ is the same as in (6.19). In (8.23) $(0,0)$ are the coordinates of $P_{k-1}$ in $\left(y_{1}^{(k)}, y_{2}^{(k)}\right)$ coordinates on $\bar{\Gamma}_{k}$ and $\left(a_{k-1}, 0\right)$ are the coordinates of $P_{k-1}$ in $\left(y_{1}^{(k-1)}, y_{2}^{(k-1)}\right)$ coordinates on $\bar{\Gamma}_{k-1}$. The Fredholmity condition (see (6.22)) has the form

$$
s \neq 1+\frac{2 \pi}{\alpha_{k-1}} \operatorname{Re} \gamma_{k-1,0}^{(1)}+\frac{\pi p}{\alpha_{k-1}}, \quad p \in \mathbf{Z}, 1 \leq k \leq N .
$$

The index of the oblique derivative problem has the following form (see (6.30)):

$$
\begin{aligned}
\kappa= & \left.\frac{1}{\pi} \sum_{k=1}^{m} \Delta \arg \left(c_{1 k}\left(y_{1}^{(k)}\right)+i c_{2 k}\left(y_{1}^{(k)}\right)\right)\right|_{y_{1}^{(k)}=a_{k}} ^{0} \\
& -2 \sum_{k-1}^{N} \operatorname{Re} \gamma_{k-1,0}^{(1)}+\sum_{k=1}^{N} p_{k-1},
\end{aligned}
$$

where $p_{k-1}$ are integers such that

$$
2 \operatorname{Re} \gamma_{k-1,0}+1+\left(\alpha_{k-1} / \pi\right)(1-s)=p_{k-1}+\delta_{k-1},
$$

$0<\delta_{k-1}<1$. We used in $(8.26)$ that

$$
\left.\Delta \arg \frac{c_{1 k}\left(y_{1}^{(k)}\right)+i c_{2 k}\left(y_{1}^{(k)}\right)}{c_{1 k}\left(y_{1}^{(k)}\right)-i c_{2 k}\left(y_{1}^{(k)}\right)}\right|_{a_{k}} ^{0}=\left.2 \Delta \arg \left(c_{k 1}\left(y_{1}^{(k)}\right)+i c_{2 k}\left(y_{1}^{(k)}\right)\right)\right|_{a_{k}} ^{0} .
$$

Denote by $c_{0}$ the following closed curve in the complex plane: take the images of $c_{1 k}\left(y_{1}^{(k)}\right)+i c_{2 k}\left(y_{1}^{(k)}\right), 0 \leq y_{1}^{(k)} \leq a_{k}, 1 \leq k \leq N$, and connect the point $c_{1, k-1}\left(a_{k-1}\right)+i c_{2, k-1}\left(a_{k-1}\right)$ with $c_{1 k}(0)+i c_{2 k}(0)$ by the line segment if $\left|\operatorname{Re} \gamma_{k-1,0}^{(1)}\right|<\frac{1}{2}$ and by the "half-circle" drawn counterclockwise if $\operatorname{Re} \gamma_{k-1,0}^{(1)}=\frac{1}{2}$. Let $\kappa_{1}$ be the winding number of this curve. We have

$$
\kappa_{1}=\left.\frac{1}{2 \pi} \sum_{k=1}^{N} \Delta \arg \left(c_{1 k}\left(y_{1}^{(k)}\right)+i c_{2 k}\left(y_{1}^{(k)}\right)\right)\right|_{a_{k}} ^{0}-\sum_{k=1}^{N} \operatorname{Re} \gamma_{k-1,0}^{(1)},
$$

where the direction' of $\partial \mathscr{D}$ is clockwise. Then

$$
\kappa=2 \kappa_{1}+\sum_{k=1}^{N} p_{k-1}
$$

where $\kappa_{1}, p_{k-1}$ are defined in (8.27), (8.29).

Remark 8.1. It was shown at the end of $\S 7$ that ind $\mathscr{A}_{S, N_{1}}=$ ind $\mathscr{A}_{2, N_{1}+N_{2}}$ for any $N_{2} \geq 0$ where $\mathscr{A}_{s, N}$ is the operator acting from $H_{s, N}(\mathscr{D})$ to $\mathscr{H}_{s, N}$ and defined by the left-hand sides of (1.1), (1.2); i.e., the index of $\mathscr{A}_{S, N_{1}+N_{2}}$ is independent 
of $N_{2}$. We shall show that this property is equivalent to the regularity in the weight norms of the solution of (1.1), (1.2). It was shown in Proposition 7.5 that the regularity implies that the index is independent of $N_{1}$. Now we shall prove the converse statement.

Proposition 8.1. Let the condition of Theorem 7.2 be satisfied and $u \in H_{s, N_{1}}(\mathscr{D})$ be a solution of (1.1), (1.2) with $F=\left\{f, h_{k j}, 1 \leq k \leq N, 1 \leq j \leq m\right\} \in$ $\mathscr{H}_{s, N_{1}+N_{2}}, N_{2}>0$. Then also $u \in H_{s, N_{1}+N_{2}}(\mathscr{D})$.

Proof. Since $H_{s, N_{1}+N_{2}}(\mathscr{D}) \subset H_{s, N_{1}}(\mathscr{D})$ we have that

$$
\operatorname{dim} \operatorname{ker} \mathscr{A}_{s, N_{1}+N_{2}} \leq \operatorname{dim} \operatorname{ker} \mathscr{A}_{s, N_{1}} .
$$

Also dim coker $\mathscr{A}_{s, N_{1}+N_{2}} \geq \operatorname{dim}$ coker $\mathscr{A}_{s, N_{1}}$ since $\mathscr{H}_{s, N_{1}}^{*} \subset \mathscr{H}_{s, N_{1}+N_{2}}^{*}$. Therefore

$$
\text { ind } \mathscr{A}_{s, N_{1}}=\text { ind } \mathscr{A}_{s, N_{1}+N_{2}}
$$

implies that

$$
\operatorname{dim} \operatorname{ker} \mathscr{A}_{s, N_{1}}=\operatorname{dim} \operatorname{ker} \mathscr{A}_{s, N_{1}+N_{2}}, \quad \operatorname{dim} \operatorname{coker} \mathscr{A}_{s, N_{1}}=\operatorname{dim} \operatorname{coker} \mathscr{A}_{s, N_{1}+N_{2}} .
$$

We have

$$
\mathscr{A}_{s, N_{1}} u=F \text {, }
$$

where $u \in H_{s, N_{1}}(\mathscr{D}), F \in \mathscr{H}_{s, N_{1}+N_{2}} \subset \mathscr{H}_{s, N_{1}}$. Since $F \in \operatorname{Im} \mathscr{A}_{s, N_{1}}$ we have that $F$ is orthogonal to coker $\mathscr{A}_{s, N_{1}}$. But coker $\mathscr{A}_{s, N_{1}}=\operatorname{coker} \mathscr{A}_{s, N_{1}+N_{2}}$ so that $F$ is orthogonal to coker $\mathscr{A}_{s, N_{1}+N_{2}}$; that is, $F \in \operatorname{Im} \mathscr{A}_{s, N_{1}+N_{2}}$. This means that there exists $v \in H_{s, N_{1}+N_{2}}(\mathscr{D})$ such that

$$
\mathscr{A}_{s, N_{1}+N_{2}} v=F \text {. }
$$

Therefore $\mathscr{A}_{s, N_{1}}(u-v)=0$; that is, $u-v \in \operatorname{ker} \mathscr{A}_{s, N_{1}}$. Since $\operatorname{ker} \mathscr{A}_{s, N_{1}}=$ $\operatorname{ker} \mathscr{A}_{s, N_{1}+N_{2}} \forall N_{2}$ we have that $u-v \in H_{s, N_{1}+N_{2}}(\mathscr{D})$; that is, $u \in H_{s, N_{1}+N_{2}}(\mathscr{D})$.

Analogously, we can prove the following proposition.

Proposition 8.2. Let the condition of Theorem 7.2 be satisfied and $\left(s_{1}, s_{2}\right)$ be two adjacent numbers from the exceptional set $\Sigma_{B}$ corresponding to the boundary value problem (1.1), (1.2), that is, $\mathscr{A}_{s, N_{1}}$ is Fredholm for any $s_{1}<s<s_{2}$. Assume that $u \in H_{s^{\prime}, N_{1}}(\mathscr{D}), F \in \mathscr{H}_{s^{\prime \prime}, N_{1}}$ where $s_{1}<s^{\prime}<s^{\prime \prime}<s_{2}, \mathscr{A}_{s, N_{1}} u=F$. Then $u \in H_{s^{\prime \prime}, N_{\mathrm{l}}}(\mathscr{D})$.

Proof. Since ind $\mathscr{A}_{s, N_{1}}$ is constant for $s_{1}<s<s_{2}$ and since $\operatorname{dim} \operatorname{ker} \mathscr{A}_{s, N_{1}}$ is decreasing and dim coker $\mathscr{A}_{s, N_{1}}$ is increasing when $s$ is increasing, we have that $\operatorname{dim} \operatorname{ker} \mathscr{A}_{s^{\prime}, N_{1}}=\operatorname{dim} \operatorname{ker} \mathscr{A}_{s^{\prime \prime}, N_{1}}, \operatorname{dim} \operatorname{coker} \mathscr{A}_{s^{\prime}, N_{1}}=\operatorname{dim} \operatorname{coker} \mathscr{A}_{s^{\prime \prime}, N_{1}}$. The rest of the proof is the same as the proof of Proposition 8.1.

\section{THE CASE OF MULTIPLE ROOTS}

In this section we shall drop the restriction that the roots of $A_{0}(x, \xi)$ are simple and shall assume only that $A_{0}(x, \xi)$ is a properly elliptic operator. In 
$y^{(k)}=\left(y_{1}^{(k)}, y_{2}^{(k)}\right)$ coordinates in a neighborhood of $\Gamma_{k}$ we shall replace operator (4.5) by the following operator:

(9.1) $\quad R_{k j}^{\prime} c_{k j}=\frac{1}{(2 \pi)^{2} i} \int_{-\infty}^{\infty} \int_{\Gamma^{+}} \frac{e^{-i w y_{2}^{(k)}} w^{j-1}\left|\eta_{1}^{(k)}\right|^{m-j} d w}{A_{k 0}^{+}\left(y^{(k)}, \eta_{1}^{(k)}, w\right) \Lambda_{-, k}^{s-1 / 2}\left(y^{(k)}, \eta_{1}^{(k)}, w\right)}$

$$
\cdot \tilde{c}_{k j}^{+}\left(\eta_{1}^{(k)}\right) e^{-i y_{1}^{(k)} \eta_{1}^{(k)}} d \eta_{1}^{(k)}
$$

where $A_{k 0}^{+}, \Gamma_{+}$are the same as in (1.4). Then instead of (4.27) we obtain (9.2)

$$
\begin{aligned}
& p_{\Gamma_{k}} \Lambda_{1, k}^{s-m_{k j}-1 / 2} B_{k j}\left(p_{\mathscr{D}} \sum_{n=1}^{N} \sum_{r=1}^{m} \psi_{n} R_{n r}^{\prime} c_{n r}\right) \\
& =p_{\Gamma_{k}}\left(\sum_{r=1}^{m} b_{k j r}^{1} c_{k r}^{+}+\sum_{r=1}^{m} \varphi_{k 1} M_{k j, k-1, r}^{\prime} c_{k-1, r}\right. \\
& \left.\quad+\sum_{r=1}^{m} \varphi_{k 2} M_{k j, k+1, r}^{\prime} c_{k+1, r}+\sum_{n=1}^{N} \sum_{r=1}^{m} T_{k j n r} c_{n r}\right) \\
& =h_{k j}^{(1)}-p_{\Gamma_{k}} \Lambda_{1, k}^{s-m_{k j}-1 / 2} B_{k j} A_{0}^{(-1)} l_{0} g,
\end{aligned}
$$

where $b_{k j r}^{1}$ are pseudodifferential operators in $\mathbf{R}^{1}$ with symbols

$$
\begin{aligned}
b_{k j r}^{1}\left(y_{1}^{(k)}, \eta_{1}^{(k)}\right)= & \frac{1}{2 \pi i} \int_{\Gamma_{+}} \frac{B_{k j 0}\left(y_{1}^{(k)}, 0, \eta_{1}^{(k)}, w\right) w^{r-1} d w}{A_{k 0}^{+}\left(y_{1}^{(k)}, 0, \eta_{1}^{(k)}, w\right) \Lambda_{-, k}^{s-1 / 2}\left(y_{1}^{(k)}, 0, \eta_{1}^{(k)}, w\right)} \\
& \cdot\left|\eta_{1}^{(k)}\right|^{m-r} \Lambda_{1, k}^{s-m_{k j}-1 / 2}\left(y_{1}^{(k)}, \eta_{1}^{(k)}\right)
\end{aligned}
$$

and $M_{k j, k-1, r}^{\prime}, M_{k j, k+1, r}^{\prime}$ are the Mellin operators of the form (4.28), (4.29) with the following kernels:

(9.4)

$$
\begin{aligned}
& M_{k j, k-1, r}^{\prime}\left(y_{1}^{(k)}, t_{k-1}-a_{k-1}\right) \\
& =\frac{1}{(2 \pi)^{2} i} \int_{-\infty}^{\infty} \int_{\Gamma_{+}} \frac{B_{k j, k-1}\left(a_{k-1}, 0, \eta_{1}^{(k-1)}, w\right) w^{r-1}}{A_{k-1,0}^{+}\left(a_{k-1}, 0, \eta_{1}^{(k-1)}, w\right)} \\
& \quad \cdot \frac{\left|\eta_{1}^{(k)}\right|^{m-r}\left(-\eta_{1}^{(k-1)} \cos \alpha_{k-1}+w \sin \alpha_{k-1}-i 0\right)^{s-m_{k j}-1 / 2} e^{-i w y_{1}^{(k)} \sin \alpha_{k-1}}}{\Lambda_{-, k-1}^{s-1 / 2}\left(a_{k-1}, 0, \eta_{1}^{(k-1)}, w\right)} d w \\
& \cdot e^{-i \eta_{1}^{(k)}\left(-y_{1}^{(k)} \cos \alpha_{k-1}+a_{k-1}-t_{k-1}\right)} d \eta_{1}^{(k-1)}
\end{aligned}
$$


Changing $w$ to $\left|\eta_{1}^{(k)}\right| w$ and integrating in $\eta_{1}^{(k)}$ from 0 to $+\infty$ and $-\infty$ to 0 , we obtain

$\left(9.4^{\prime}\right)$

$$
\begin{aligned}
& M_{k j, k-1, r}^{\prime}\left(y_{1}^{(k)}, t_{k-1}-a_{k-1}\right)=\frac{-1}{(2 \pi)^{2}} \int_{\Gamma^{+}} \frac{B_{k j, k-1}\left(a_{k-1}, 0,1, w\right) w^{r-1}}{A_{k-1,0}^{+}\left(a_{k-1}, 0,1, w\right)} \\
& \cdot \frac{\left(-\cos \alpha_{k-1}+w \sin \alpha_{k-1}-i 0\right)^{s-m_{k j}-1 / 2} d w}{\Lambda_{-, k-1}^{s-1 / 2}\left(a_{k-1}, 0,1, w\right)\left(y_{1}^{(k-1)}\left(w \sin \alpha_{k-1}-\cos \alpha_{k-1}\right)+a_{k-1}-t_{k-1}\right)} \\
& -\frac{1}{(2 \pi)^{2}} \int_{\Gamma_{+}} \frac{B_{k j, k-1}\left(a_{k-1}, 0,-1, w\right) w^{r-1}}{A_{k-1,0}^{+}\left(a_{k-1}, 0,-1, w\right) \Lambda_{-, k-1}^{s-1 / 2}\left(a_{k-1}, 0,-1, w\right)} \\
& \cdot \frac{\left(\cos \alpha_{k-1}+w \sin \alpha_{k-1}-i 0\right)^{s-m_{k j}-1 / 2} d w}{\left(y_{1}^{(k-1)}\left(w \sin \alpha_{k-1}+\cos \alpha_{k-1}\right)-a_{k-1}+t_{k-1}\right)}
\end{aligned}
$$

Analogously,

$$
\begin{gathered}
M_{k j, k+1, r}^{\prime}\left(y_{1}^{(k)}-a_{k}, t_{k+1}\right)=\frac{-1}{(2 \pi)^{2}} \int_{\Gamma_{+}} \frac{B_{k j, k+1}(0,0,1, w) w^{r-1}}{A_{k+1,0}(0,0,1, w)} \\
\cdot \frac{\left(-\cos \alpha_{k}-w \sin \alpha_{k}+i 0\right)^{s-m_{k j}-1 / 2} d w}{\Lambda_{-, k+1}^{s-1 / 2}(0,0,1, w)\left(-\left(y_{1}^{(k)}-a_{k}\right)\left(w \sin \alpha_{k}+\cos \alpha_{k}\right)-t_{k+1}\right)} \\
+\frac{1}{(2 \pi)^{2}} \int_{\Gamma_{+}} \frac{B_{k j, k+1}(0,0,-1, w) w^{r-1}}{A_{k+1,0}^{+}(0,0,-1, w) \Lambda_{-, k+1}^{s-1 / 2}(0,0,-1, w)} \\
\cdot \frac{\left(\cos \alpha_{k}-w \sin \alpha_{k}+i 0\right)^{s-m_{k j}-1 / 2} d w}{\left(\left(y_{1}^{(k)}-a_{k}\right)\left(w \sin \alpha_{k}-\cos \alpha_{k}\right)-t_{k+1}\right)}
\end{gathered}
$$

In (9.4), $\left(9.4^{\prime}\right),(9.5)$ we use the same notation as in (4.30), (4.31) and we assume that $\alpha_{k-1} \leq \pi, \alpha_{k} \leq \pi$. For the case when the angle is larger than $\pi$ see Remark 9.1. Analogously to (4.27), the system (9.2) can be reduced to a system of the form (5.1). It follows from (5.22), (5.23), (5.24) that the system (9.2) is Fredholm iff

$$
\begin{gathered}
\operatorname{det}\left\|b_{k j r}^{1}\left(y_{1}^{(k)}, \pm 1\right)\right\|_{j, r=1}^{m} \neq 0, \quad 0 \leq y_{1}^{(k)} \leq a_{k}, 1 \leq k \leq N \\
\operatorname{det} M_{1}^{(k-1)}(z) \neq 0, \quad z=\frac{1}{2}+i \tau, \tau \in \mathbf{R}, 1 \leq k \leq N,
\end{gathered}
$$

where

$$
M_{1}^{(k-1)}(z)=\left(1-e^{2 \pi i z}\right)^{-1} \| \begin{array}{cc}
b_{+, k}^{1}-b_{-, k}^{1} e^{2 \pi i z} & -\left(m_{+, k}(z)-m_{-, k}(z)\right)\left(\frac{a_{k-1}}{a_{k}}\right)^{z}
\end{array} \mid
$$


where

$$
\begin{aligned}
b_{ \pm, k}^{1} & =\left\|b_{k j r}^{1}(0, \pm 1)\right\|_{j, r=1}^{m}, \\
b_{ \pm, k-1}^{1} & =\left\|b_{k-1, j r}^{1}\left(a_{k-1}, \pm 1\right)\right\|_{j, r=1}^{m},
\end{aligned}
$$

$m_{+, k}(z)-m_{-, k}(z)$ is the Mellin transform of $\left\|M_{k j, k-1, r}^{\prime}\left(y_{1}^{(k)},-1\right)\right\|_{j, r=1}^{m}$ in $y_{1}^{(k)}, 0<y_{1}^{(k)}<+\infty, m_{+, k}(z)$ corresponds to the first integral in $\left(9.4^{\prime}\right)$ and $m_{-, k}(z)$ to the second and $m_{+, k-1}(z)-m_{-, k-1}(z)$ is defined analogously. It follows from (5.26) that the formula for the index of the boundary value problem (1.1), (1.2) has the form

$$
\begin{aligned}
\kappa= & \left.\sum_{k=1}^{N} \frac{1}{2 \pi} \Delta \arg \operatorname{det}\left\|b_{k j r}^{1}\left(y_{1}^{(k)}, 1\right)\right\|^{-1}\left\|b_{k j r}^{1}\left(y_{1}^{(k)},-1\right)\right\|\right|_{a_{k}} ^{0} \\
& +\left.\frac{1}{2 \pi} \sum_{k=1}^{N} \frac{1}{2 \pi} \Delta \arg \operatorname{det} M_{1}^{(k-1)}(z)\right|_{1 / 2-i \infty} ^{1 / 2+i \infty} .
\end{aligned}
$$

As in $\S \S 5,6$ we can simplify conditions (9.6), (9.7) and the formula (9.10). First consider condition (9.6). Assume temporarily that $A_{k, 0}^{+}\left(y_{1}^{(k)}, 0, \pm 1, z\right)=0$ has simple zeros. Note that the set of such $A_{k, 0}^{+}$is dense in the set of all $A_{k, 0}^{+}$. Then computing (9.3) using the residues, we obtain

$$
\begin{aligned}
b_{k j r}^{1}\left(y_{1}^{(k)}, \pm 1\right)= & \sum_{p=1}^{m} B_{k j 0}\left(y_{1}^{(k)}, 0, \pm 1, \lambda_{k p}\left(y_{1}^{(k)}, 0, \pm 1\right)\right) \\
& \cdot \lambda_{k p}^{r-1}\left(y_{1}^{(k)}, 0, \pm 1\right) \Lambda_{-, k}^{1 / 2-s}\left(y_{1}^{(k)}, 0, \pm 1, \lambda_{k p}\left(y_{1}^{(k)}, 0, \pm 1\right)\right) \\
& \cdot\left(\frac{\partial A_{k 0}^{+}}{\partial z}\left(y_{1}^{(k)}, 0, \pm 1, \lambda_{k p}\left(y_{1}^{(k)}, 0, \pm 1\right)\right)\right)^{-1} \\
& \cdot \Lambda_{1, k}^{s-m_{k j}-1 / 2}\left(y_{1}^{(k)}, \pm 1\right) .
\end{aligned}
$$

There exist polynomials $a_{k n}^{ \pm}\left(y_{1}^{(k)}, w\right)$ of degree $\leq n$ with $C^{\infty}$ coefficients in $y_{1}^{(k)}$ such that

$$
\frac{1}{2 \pi i} \int_{\Gamma_{+}} \frac{a_{k, m-n}^{ \pm}\left(y_{1}^{(k)}, w\right) w^{r-1} d w}{A_{k 0}^{+}\left(y_{1}^{(k)}, 0, \pm 1, w\right)}=\delta_{n r}
$$

Indeed

$$
\frac{1}{2 \pi i} \int_{\Gamma_{+}} \frac{w^{m-n+r-1} d w}{A_{k 0}^{+}}=a_{n r}^{(0)}\left(y_{1}^{(k)}\right),
$$

where $a_{n r}^{(0)}=0$ for $r<n, a_{n n}^{(0)}=-1$. To obtain (9.12) one should apply the inverse of $\left\|a_{n r}^{(0)}\right\|$ to (9.13). In the case when $A_{k 0}^{+}$has simple zeros (9.12) has 
the following form:

$$
\begin{aligned}
\sum_{p=1}^{m} a_{k, m-n}^{ \pm}\left(y_{1}^{(k)}, \lambda_{k p}\left(y_{1}^{(k)}, 0, \pm 1\right)\right) \lambda_{k p}^{r-1}\left(y_{1}^{(k)}, 0, \pm 1\right) & \\
& \cdot\left(\frac{\partial A_{k 0}^{+}}{\partial z}\left(y_{1}^{(k)}, 0, \pm 1, \lambda_{k p}\right)\right)^{-1}=\delta_{n r}
\end{aligned}
$$

that is,

$$
\left\|a_{k, m-n}^{ \pm}\left(y_{1}^{(k)}, \lambda_{k p}\left(y_{1}^{(k)}, 0, \pm 1\right)\right)\right\|_{n, p=1}^{m}
$$

is the inverse matrix to

$$
\left\|\lambda_{k p}^{r-1}\left(y_{1}^{(k)}, 0, \pm 1\right)\left(\frac{\partial A_{k 0}^{+}}{\partial z}\left(y_{1}^{(k)}, 0, \pm 1, \lambda_{k p}\right)\right)^{-1}\right\|_{p, r=1}^{m} .
$$

Using (9.14) one can rewrite (9.11) in the following form:

(9.15) $b_{k j r}^{1}\left(y_{1}^{(k)}, \pm 1\right)=\sum_{p=1}^{m} \Lambda_{1, k}^{s-m_{k j}-1 / 2}\left(y_{1}^{(k)}, \pm 1\right) b_{k j p}^{(0)}\left(y_{1}^{(k)}, \pm 1\right) c_{k p r}\left(y_{1}^{(k)}, \pm 1\right)$,

where $b_{k j p}^{(0)}\left(y_{1}^{(k)}, \pm 1\right)$ are the same as in (1.4), that is, $b_{k j p}^{(0)}$ is the Lopatinsky matrix, and

$$
c_{k p r}\left(y_{1}^{(k)}, \pm 1\right)=\frac{1}{2 \pi i} \int_{\Gamma_{+}} \frac{a_{k, m-p}^{ \pm}\left(y_{1}^{(k)}, w\right) w^{r-1} d w}{A_{k 0}^{+}\left(y_{1}^{(k)}, 0, \pm 1, w\right) \Lambda_{-, k}^{s-1 / 2}\left(y_{1}^{(k)}, 0, \pm 1, w\right)} .
$$

Using the residues we obtain from (9.16) that

$$
\text { (9.17) } \operatorname{det}\left\|c_{k p r}\left(y_{1}^{(k)}, \pm 1\right)\right\|_{p, r=1}^{m}=\prod_{p=1}^{m} \Lambda_{-, k}^{1 / 2-s}\left(y_{1}^{(k)}, 0, \pm 1, \lambda_{k p}\left(y_{1}^{(k)}, 0, \pm 1\right)\right) \text {. }
$$

Taking the limit we have that equations (9.15) and (9.17) hold for any $A_{k 0}^{+}\left(y_{1}^{(k)}, 0, \pm 1, w\right)$.

It follows from (9.15) that condition (9.6) is equivalent to condition (1.5), the Shapiro-Lopatinsky condition. It also follows from (9.15), (9.16) (cf. (6.1)(6.11)) that

$$
\begin{aligned}
\left.\sum_{k=1}^{N} \frac{1}{2 \pi} \Delta \arg \operatorname{det}\left\|b_{k j r}^{1}\left(y_{1}^{(k)},+1\right)\right\|^{-1}\left\|b_{k j r}^{1}\left(y_{1}^{(k)},-1\right)\right\|\right|_{a_{k}} ^{0} \\
=\left.\sum_{k=1}^{N} \frac{1}{2 \pi} \Delta \arg \operatorname{det}\left\|b_{k j r}^{(0)}\left(y_{1}^{(k)},+1\right)\right\|^{-1}\left\|b_{k j r}^{(0)}\left(y_{1}^{(k)},-1\right)\right\|\right|_{a_{k}} ^{0} \\
\quad+\sum_{k=1}^{N} \sum_{j=1}^{m} m_{k j}+\frac{1}{2 \pi}\left(\frac{1}{2}-s\right) \sum_{k=1}^{N} \sum_{p=1}^{m}\left(\operatorname{Re} \beta_{k, p+m}-\operatorname{Re} \beta_{k, p}+2 \pi\right),
\end{aligned}
$$

where $\beta_{k, p}$ are the same as in (6.10), $1 \leq p \leq 2 m$. 
Now consider (9.8). Analogously to (9.15) we have

$$
\begin{gathered}
m_{+, k}(z)=b_{+, k}^{1} \Delta_{k 1}^{1}(z), \quad m_{-, k}(z)=b_{-, k}^{1} \Delta_{k 2}^{1}(z), \\
m_{+, k-1}(z)=b_{+, k-1}^{1} e^{2 \pi i z}\left(\Delta_{k 1}^{1}(z)\right)^{-1}, \\
m_{-, k-1}(z)=b_{-, k-1}^{1} e^{2 \pi i z}\left(\Delta_{k 2}^{1}(z)\right)^{-1}
\end{gathered}
$$

where $b_{ \pm, k}^{1}, b_{ \pm, k-1}^{1}$ are the same as in (9.9) and

$$
\Delta_{k 1}^{1}(z)=\left\|\frac{1}{2 \pi i} \int_{\Gamma_{+}} \frac{a_{k, m-n}^{+}(0, w) w^{p-1} e^{z \ln \left(\cos \alpha_{k-1}+w \sin \alpha_{k-1}\right)} d w}{A_{k 0}^{+}(0,0,+1, w)}\right\|_{n, p=1}^{m},
$$

$$
\Delta_{k 2}^{1}(z)=\left\|\frac{1}{2 \pi i} \int_{\Gamma_{+}} \frac{a_{k, m-n}^{-}(0, w) w^{p-1} e^{z \ln \left(\cos \alpha_{k-1}-w \sin \alpha_{k-1}\right)} d w}{A_{k 0}^{+}(0,0,-1, w)}\right\|_{n, p=1}^{m},
$$

where

$$
0<\arg \ln \left(\cos \alpha_{k-1} \pm w \sin \alpha_{k-1}\right)<2 \pi, \quad \operatorname{Im} w<0
$$

Denote

$$
\Delta_{k 3}^{1}(z)=\left(1-e^{2 \pi i z}\right)^{-1}\left\|\begin{array}{cc}
I & -\Delta_{k 1}^{1}(z)\left(\frac{a_{k-1}}{a_{k}}\right)^{z}
\end{array}\right\| .
$$

We have using (9.12) that (cf. (5.36)):

$$
\operatorname{det} \Delta_{k 3}^{1}(z)=\left(1-e^{2 \pi i z}\right)^{-1} \prod_{j=1}^{m}\left(-1+e^{i\left(2 \pi-\beta_{k, j+m}+\beta_{k j}\right) z}\right) .
$$

Assuming that (9.25) is not zero for $z=\frac{1}{2}+i \tau, \tau \in \mathbf{R}$, we obtain analogously to (5.34), (5.48) that (9.7) is equivalent to the following condition:

(9.26) $\operatorname{det} M_{k-1,0}^{1}\left(z-s+\frac{1}{2}\right) \neq 0, \quad z=\frac{1}{2}+i \tau,-\infty<\tau<+\infty, 1 \leq k \leq N$, where

$$
\begin{aligned}
M_{k-1,0}^{1}(z)= & -\left(b_{+, k-1}^{(0)}\right)^{-1}\left\|e^{-i \pi m_{k-1, j}} \delta_{j p}\right\| b_{-, k-1}^{(0)} \\
& +e^{2 \pi i z}\left(\Delta_{k 1}^{1}(z)\right)^{-1}\left(b_{+, k}^{(0)}\right)^{-1}\left\|e^{-i \pi m_{k j}} \delta_{j p}\right\| b_{-, k}^{(0)} \Delta_{k 2}^{1}(z),
\end{aligned}
$$

$b_{ \pm, k}^{(0)}=\left\|b_{k j p}^{(0)}(0, \pm 1)\right\|, b_{ \pm, k-1}^{(0)}=\left\|b_{k j p}^{(0)}\left(a_{k-1}, \pm 1\right)\right\|$, and $\Delta_{k 1}^{1}(z), \Delta_{k 2}^{1}(z)$ are defined in (9.21), (9.22).

Remark 9.1. We have assumed that (9.25) is not zero for all $z=\frac{1}{2}+i \tau$, as in the case when $A_{0}(x, \xi)$ is real. Otherwise, analogously to Remarks 2.2 and 5.1, we need to change operator $R_{k j}^{1} c_{k j}$, replacing $\tilde{c}_{k j}^{+}\left(\eta_{1}^{(k)}\right)$ by $\sum_{r=1}^{m} d_{k j r}\left(\eta_{1}^{(k)}\right) \tilde{c}_{k r}^{+}\left(\eta_{1}^{(k)}\right)$, 
where $\operatorname{deg}_{\eta_{1}^{(k)}} d_{k j r}\left(\eta_{1}^{(k)}\right)=0$ (cf. (2.84)). Also we assumed in (9.1) that $\alpha_{k-1} \leq$ $\pi, \alpha_{k} \leq \pi$. Otherwise (cf. Remarks 2.1 and 4.1) we consider the operator

$$
G_{k j}^{1} c_{k j}=\int_{0}^{a_{k}} G_{k j}^{1}\left(y^{(k)}, y_{2}^{(k)}, y_{1}^{(k)}-t\right) c_{k j}(t) d t
$$

where

$$
G_{k j}^{1}\left(y^{(k)}, x_{2}, x_{1}\right)=\frac{1}{(2 \pi)^{2} i} \int_{-\infty}^{\infty} \int_{\Gamma_{+}} \frac{e^{-i w x_{2}-i x_{1} \eta_{1}^{(k)}} w^{j-1}\left|\eta_{1}^{(k)}\right|^{m-j} d w}{A_{k 0}^{+}\left(y^{(k)}, \eta_{1}^{(k)}, w\right)} d \eta_{1}^{(k)}
$$

Integrating in $\eta_{1}^{(k)}$, we obtain analogously to $\left(9.4^{\prime}\right)$ :

$$
\begin{aligned}
G_{k j}^{1}\left(y^{(k)}, x_{2}, x_{1}\right)= & \frac{-1}{(2 \pi)^{2}} \int_{\Gamma_{+}} \frac{w^{j-1} d w}{A_{k 0}^{+}\left(y_{1}^{(k)}, 1, w\right)\left(w x_{2}+x_{1}\right)} \\
& -\frac{1}{(2 \pi)^{2}} \int_{\Gamma_{+}} \frac{w^{j-1} d w}{A_{k 0}^{+}\left(y^{(k)},-1, w\right)\left(w x_{2}-x_{1}\right)} .
\end{aligned}
$$

(9.30) gives an analytic continuation of $G_{k j}^{1}\left(y^{(k)}, x_{2}, x_{1}\right)$ for any $\left(x_{1}, x_{2}\right) \neq$ $(0,0)$. Analogously to $(2.77)$ we can find the Fourier transform $\widetilde{G_{k j}^{1}}\left(y^{(k)}, \xi_{2}, \xi_{1}\right)$ of $G_{k j}^{1}\left(y^{(k)}, x_{2}, x_{1}\right)$ in $\left(x_{1}, x_{2}\right)$ :

$$
\begin{aligned}
\widetilde{G_{k j}^{1}}\left(y^{(k)}, \xi_{2}, \xi_{1}\right)= & \frac{1}{2 \pi} \int_{\Gamma_{+}} \frac{w^{j-1} d w}{A_{k 0}^{+}\left(y^{(k)}, 1, w\right)\left(\xi_{2}-w \xi_{1}\right)} \\
& +\frac{1}{2 \pi} \int_{\Gamma_{+}} \frac{w^{j-1} d w}{A_{k 0}^{+}\left(y^{(k)},-1, w\right)\left(\xi_{2}+w \xi_{1}\right)}
\end{aligned}
$$

We shall replace $(9.1)$ by

$$
R_{k j}^{\prime \prime} c_{k j}=p_{\mathscr{D}} \Lambda_{-, k}^{1 / 2-s}\left(y^{(k)}, i \frac{\partial}{\partial y^{(k)}}\right) G_{k j}^{1} c_{k j} .
$$

Analogously to Remarks 2.1 and 4.1, we have that all results remain valid for $\pi<\alpha_{k}<2 \pi$.

We shall formulate our final result:

Theorem 9.1. Operator $\mathscr{A}_{s}$ defined by the boundary value problem (1.1), (1.2) is Fredholm iff the Shapiro-Lopatinsky condition (1.5) and the "corner condition" (9.26) are satisfied. The index of $\mathscr{A}_{s}$ is given by formula (9.10) or by the following formula when $A_{0}(x, \xi)$ is real:

$$
\begin{aligned}
\text { ind } \mathscr{A}_{s}=\left.\sum_{k=1}^{N} \frac{1}{2 \pi} \Delta \arg \operatorname{det}\left\|b_{k j r}^{(0)}\left(y_{1}^{(k)},+1\right)\right\|^{-1}\left\|b_{k j r}^{(0)}\left(y_{1}^{(k)},-1\right)\right\|\right|_{a_{k}=0} ^{0} \\
+\sum_{k=1}^{N}\left(\sum_{p=1}^{m} \frac{m_{k-1, p}+m_{k, p}-1}{2}+\left.\frac{1}{2 \pi} \Delta \arg \operatorname{det} M_{k-1,0}^{1}(z)\right|_{-0-i \infty} ^{-0+i \infty}+m_{k-1}^{(s)}\right),
\end{aligned}
$$


where $b_{k j r}^{(0)}$ are defined in (1.5), $M_{k-1,0}^{1}(z)$ is defined in (9.27), $\left|m_{k-1}^{(s)}\right|$ is the number of zeros of $\operatorname{det} M_{k-1,0}^{1}(z)=0$ between $\operatorname{Re} z=-0$ and $\operatorname{Re} z=1-s$, $m_{k-1}^{(s)}>0$ when $1-s>0$, and $m_{k-1}^{(s)}<0$ when $1-s<0$.

\section{REFERENCES}

1. H. O. Cordes, Pseudodifferential operators on a half-line, J. Math. Mech. 18 (1968/69), 893908.

2. M. Dauge, Régularités et singularités des solutions de problèmes aux limites elliptiques sur des domains singuliers de type à coins, Thèse, Université de Nantes, 1986.

3. G. I. Eskin, Boundary value problems for elliptic pseudodifferential equations, Transl. Math. Monographs, vol. 52, Amer. Math. Soc., Providence, R.I., 1981.

4. _ The conjugacy problem for equations of principal type with two independent variables, Trans. Moscow Math. Soc. 21 (1970), 263-316.

5. __ Boundary value problems for second order elliptic equations in domains with corners, Proc. Sympos. Pure Math., vol. 43, Amer. Math. Soc., Providence, R.I., 1985, pp. 105-131.

6. P. Grisvard, Elliptic problems in nonsmooth domains, Pitman, London, 1985.

7. V. A. Kondrat'ev and O. A. Oleinik, Boundary value problems for partial differential equations in nonsmooth domains, Russian Math. Surveys 38 (1983), 1-86.

8. V. G. Maz'ya and B. A. Plamenevskii, Weighted spaces with nonhomogeneous norms and boundary value problems in domains with conical points, Amer. Math. Soc. Transl. (2) 123 (1984), 89-107.

9. R. B. Melrose and G. Mendoza, Elliptic operators of totally characteristic type, MSRI preprint (1983).

10. K. T. Smith, Formulas to represent functions by their derivatives, Math. Ann. 188 (1970), 53-77.

Department of Mathematics, University of California at los ANgeles, Los ANgeles, California 90024 